
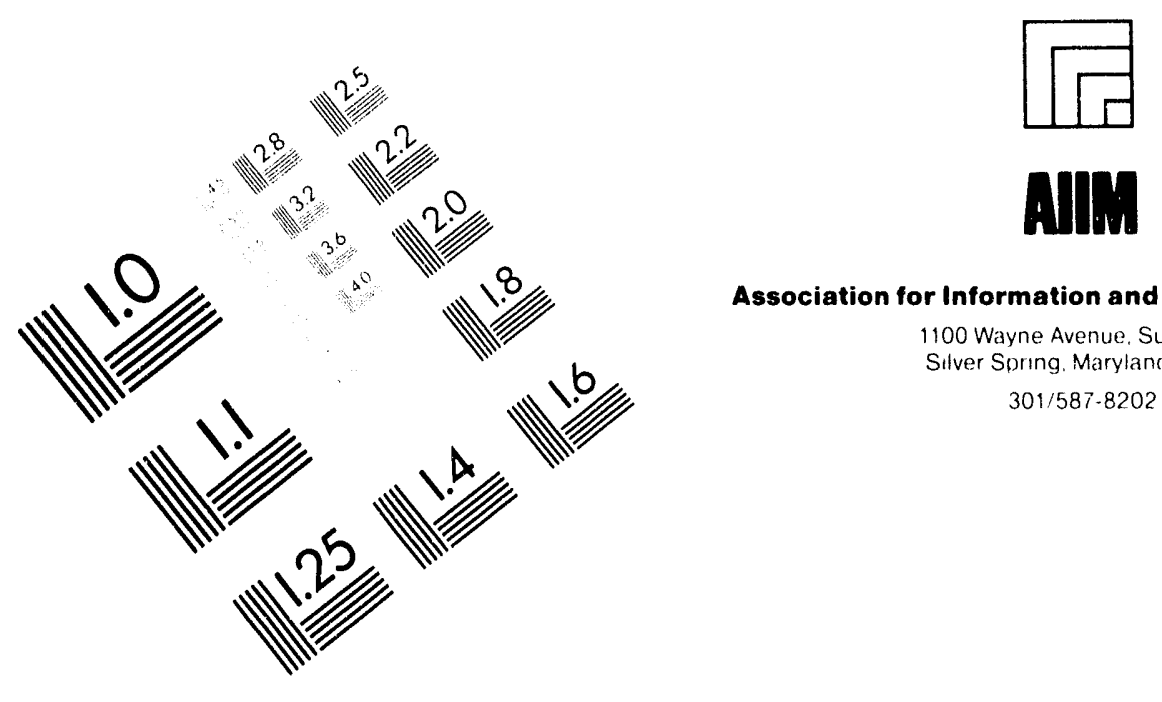

Association for Information and Image Management

1100 Wayne Avenue. Suite 1100

Silver Sorng, Maryland 20910

$301 / 587-8202$

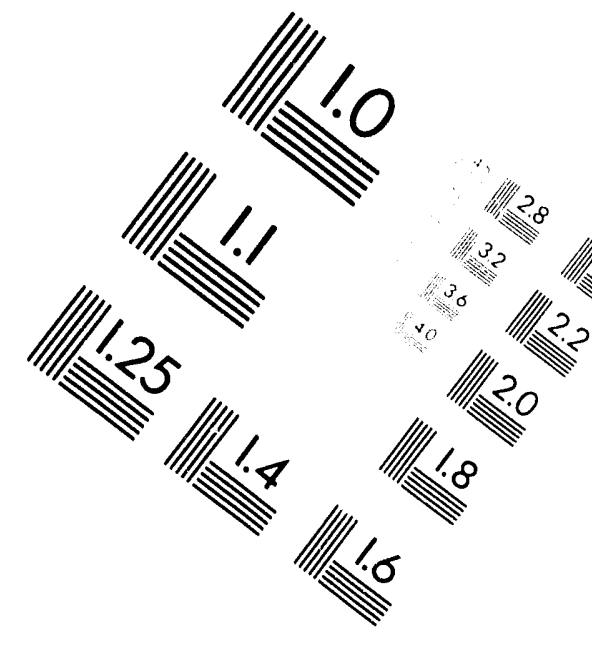

\title{
Centimeter
}

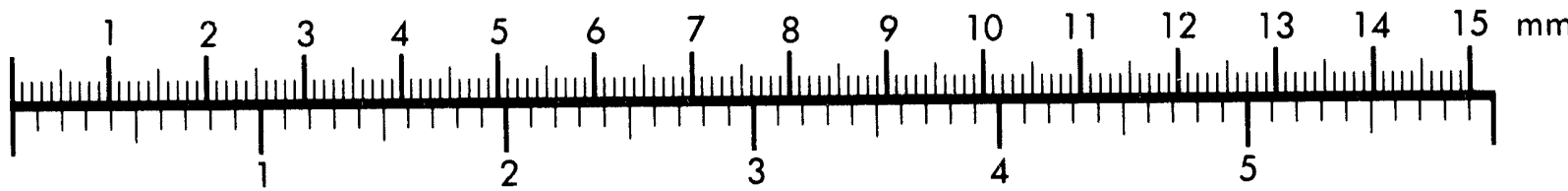

Inches
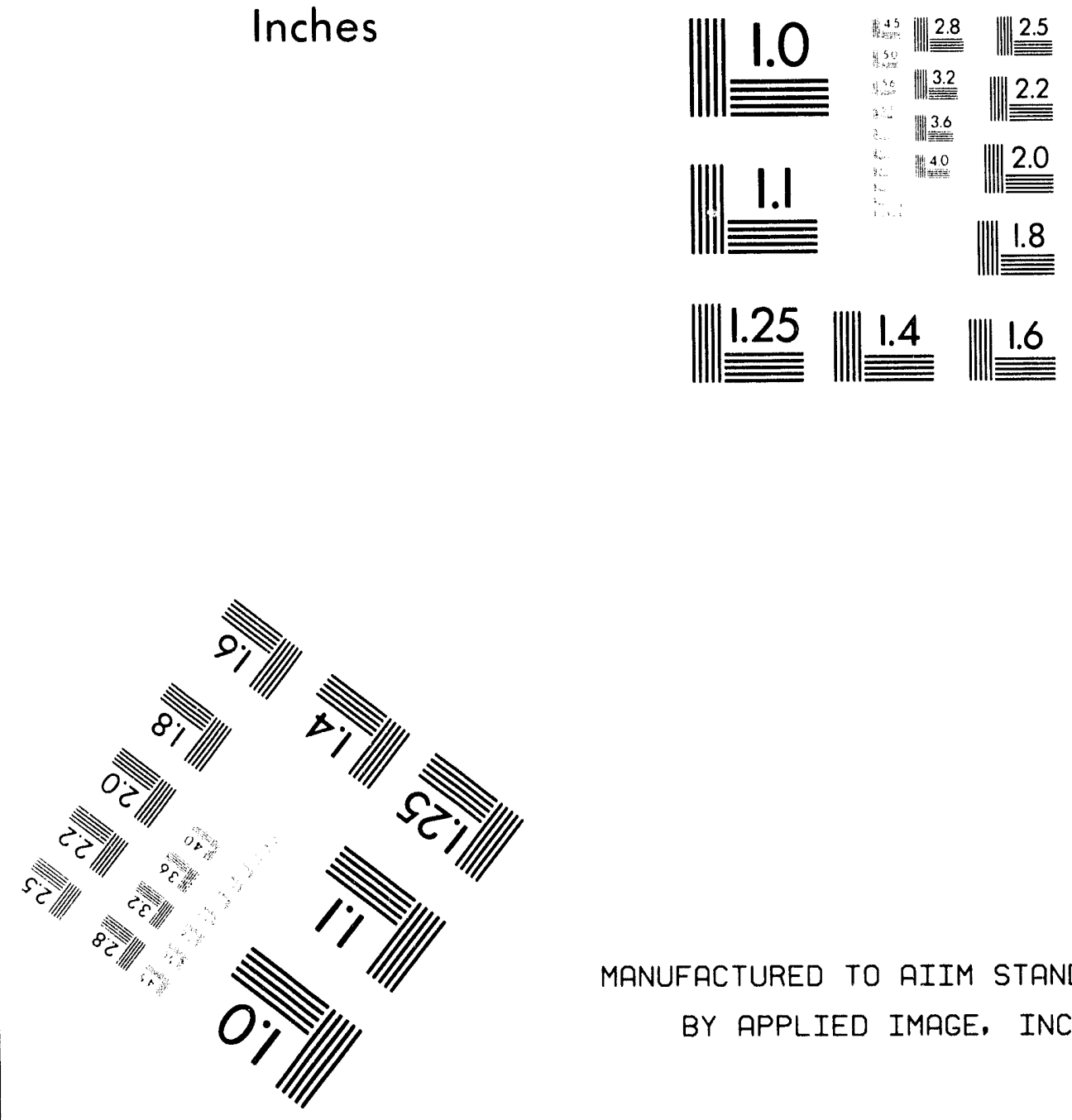

MANUFACTURED TO AIIM STANDARDS

BY APPLIED IMAGE, INC.

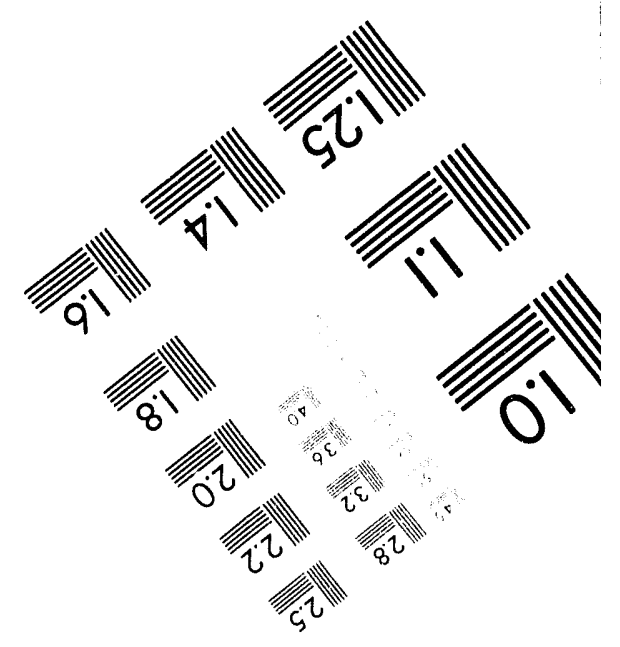



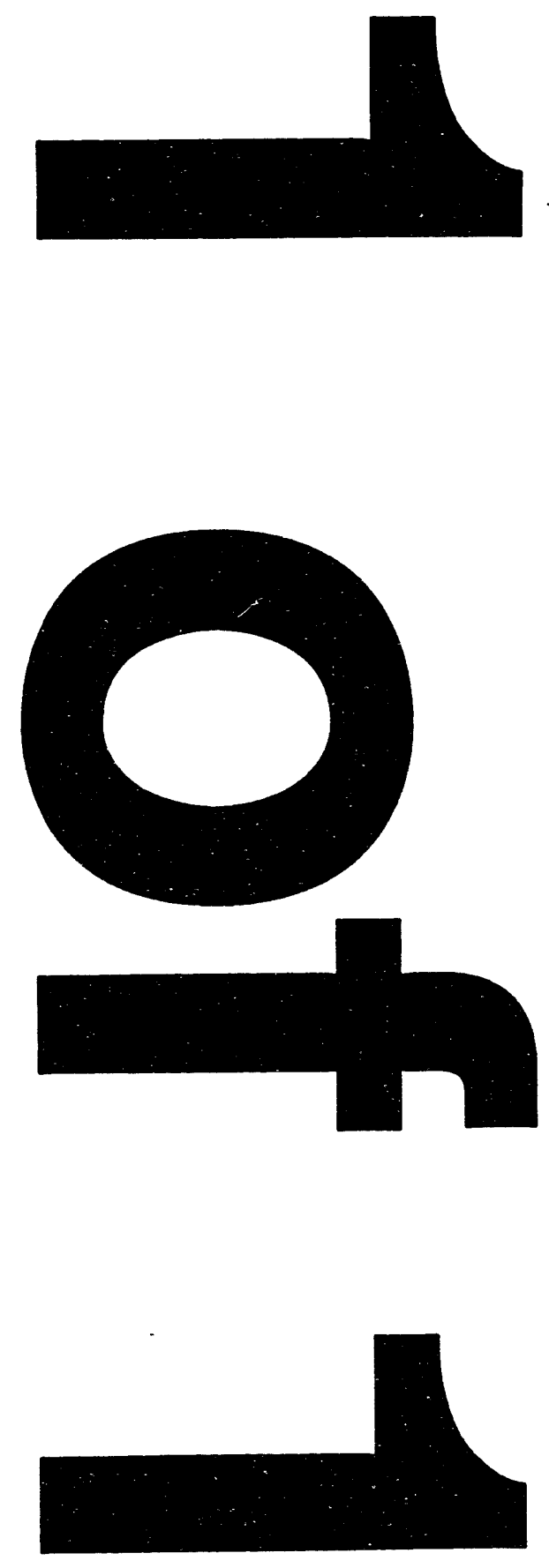
ANL/APS/TB-16

1

\section{Advanced Photon Source Accelerator Ultrahigh Vacuum Guide}

\section{Chian Liu and John Noonan}

\section{March 1994}


Argonne National Laboratory, with facilities in the states of Illinois and Idaho, is owned by the United States government, and operated by The University of Chicago under the provisions of a contract with the Department of Energy.

\section{DISCLAIMER}

This report was prepared as an account of work sponsored by an agency of the United States Government. Neither the United States Government nor any agency thereof, nor any of their employees, makes any warranty, express or implied, or assumes any legal liability or responsibility for the accuracy, completeness, or usefulness of any information, apparatus, product, or process disclosed, or represents that its use would not infringe privately owned rights. Reference herein to any specific commercial product, process, or service by trade name, trademark, manufacturer, or otherwise, does not necessarily constitute or imply its endorsement, recommendation, or favoring by the United States Government or any agency thereof. The views and opinions of authors expressed herein do not necessarily state or reflect those of the United States Government or any agency thereof.

Reproduced from the best available copy.

Available to DOE and DOE contractors from the

Office of Scientific and Technical Information

P.O. Box 62

Oak Ridge, TN 37831

Prices available from (615) 576-8401

Available to the public from the

National Technical Information Service

U.S. Department of Commerce

5285 Port Royal Road

Springfield, VA 22161 
Distribution Category: Atomic, Molecular, and Chemical Physics (UC-411)

\section{ARGONNE NATIONAL LABORATORY \\ 9700 South Cass Avenue \\ Argonne, Illinois 60439}

ANL/APS/TB-16

\section{Advanced Photon Source Accelerator Ultrahigh Vacuum Guide}

Accelerator Systems Division

Advanced Photon Source

March 1994

work sponsored by

U.S. DEPARTMENT OF ENERGY

Office of Energy Research

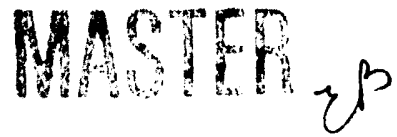

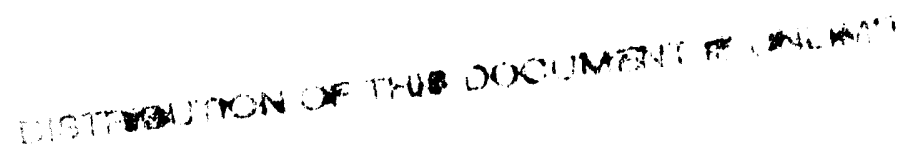




\section{CONTENTS}

1. Ultrahigh Vacuum Overview..........................................................................

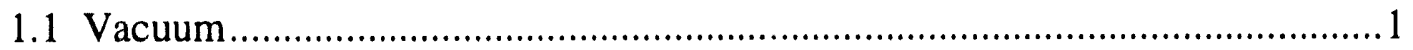

1.2 Sources of Residual Gases ..................................................................... 3

1.3 Material Selections in Ultrahigh Vacuum ........................................... 9

1.4 Pumps and Pumping Processes................................................................. 11

1.5 Common Sense in Ultrahigh Vacuum Related Work .................................. 15

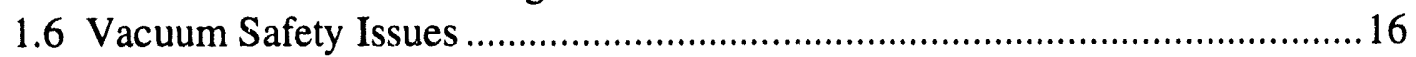

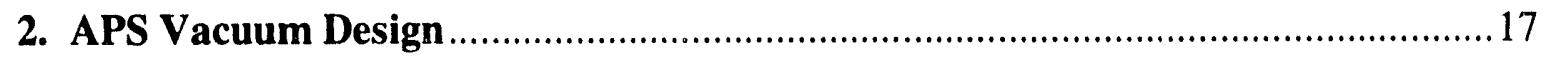

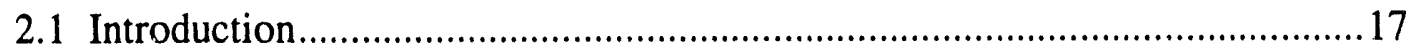

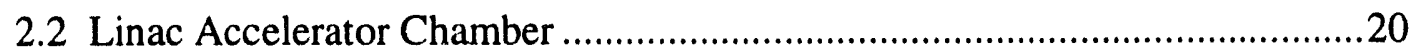

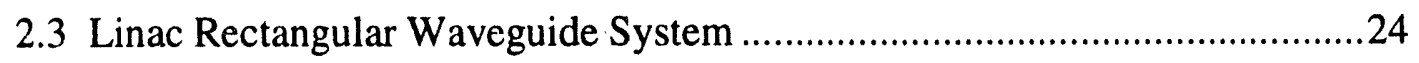

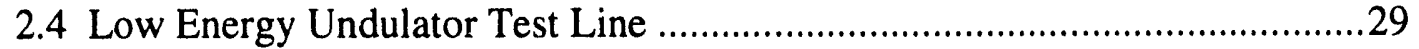

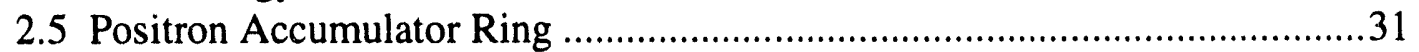

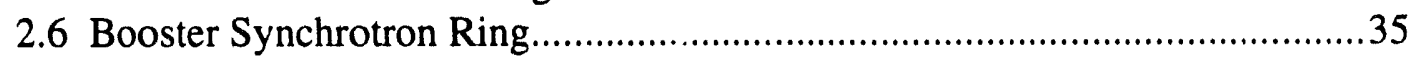

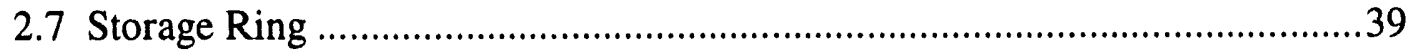

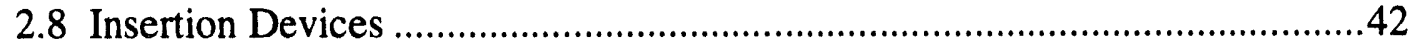

3. Cleaning Procedures for Ultrahigh Vacuum Components .......................................46

4. Summary

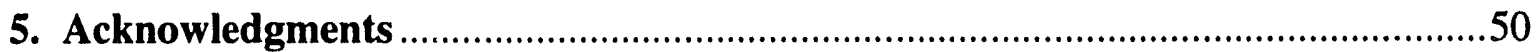

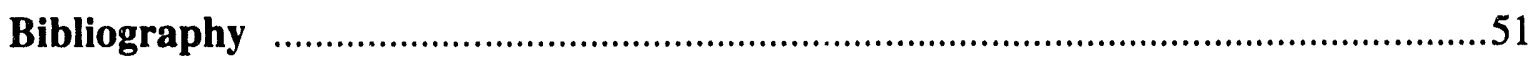

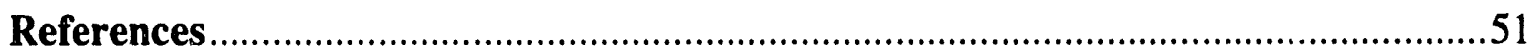

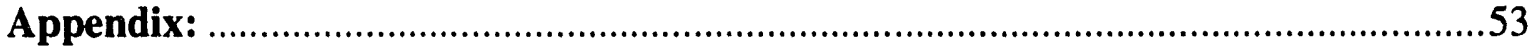

Computer Program for Calculating Pressure Profiles along PAR ………..............53 


\section{FIGURES}

Fig. 1.1 (a) Basic design of the ConFlat flange seal from Varian; (b) basic design of EVAC combination flange with a chain clamp and an outer centered aluminum knife-edge seal (EVAC International, Inc.).

Fig. 1.2 Schematic diagrams showing how a sputter ion pump works (reproduced from 1989-1990 Perkin Elmer Ultra High Vacuum Equipment Catalog, p. 29, with the permission of the copyright holder)......................13

Fig. 2.1 A schematic diagram of the linac accelerator disc-loaded waveguide

Fig. 2.2 (a) A model of the top half of two linac cavity cells used in Monte Carlo calculations. (b) A demonstration of random paths of the generated molecules in a Monte Carlo simulation.

Fig. 2.3 Pressure distribution along a linac accelerator chamber after 10 hours and 48 hours of ion pump pumping.

Fig. 2.4 A schematic drawing of the Sector 2 rectangular waveguide network

Fig. 2.5 A drawing of the HP $90^{\circ}$ waveguide bend 25

Fig. 2.6 Pressure distribution along the Sector 2 rectangular waveguide network

Fig. 2.7 Pressure distribution along the SLED branch of the Sector 2 waveguide network.

Fig. 2.8 The pressure distribution from the waveguide to the ion pump. ...............28

Fig. 2.9 Layout of the low energy undulator test line .......................................29

Fig. 2.10 Pressure distribution along the low energy undulator test line ..................30

Fig. 2.11 Layout of the PAR to the positron linac and the booster ring....................31

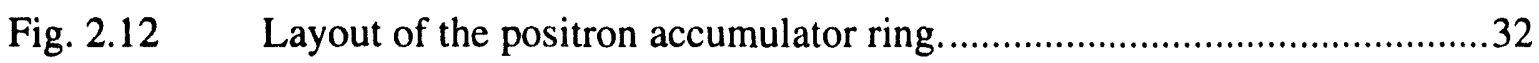

Fig. 2.13 Pressure distribution along the positron accumulator ring .........................34

Fig. 2.14 Layout of the booster synchrotron ring and the configuration of a normal cell. 
Fig. 2.15 Pressure distribution along the bottom half of the booster ring................37

Fig. 2.16 (a) A top view of the crotch-absorber chamber and (b) its side view showing the relative position of the crotch absorber with the photon beam

Fig. 2.17 The insertion device vacuum chamber cross section.........................42

Fig. 2.18 Pressure distribution along one sector of the storage ring.....................44

Fig. 2.19 Pressure profile across the insertion chamber for two designs with different dimensions. 


\section{TABLES}

Table 1.1 Gas density and mean free path under various environments for air at $25^{\circ} \mathrm{C}$ .2

Table 1.2 Coefficient of linear expansion $\alpha$ for some widely used materials ............ 5 


\title{
ADVANCED PHOTON SOURCE ACCELERATOR
}

\section{ULTRAHIGH VACUUM GUIDE}

\author{
Chian Liu and John Noonan
}

\begin{abstract}
In this document we summarize the following: (i) an overview of basic concepts of ultrahigh vacuum needed for the APS project, (ii) a description of vacuum design and calculations for major parts of APS, including linac, linac waveguide, low energy undulator test line, positron accumulator ring (PAR), booster synchrotron ring, storage ring, and insertion devices, and (iii) cleaning procedures of ultrahigh vacuum (UHV) components presently used at APS.
\end{abstract}

\section{ULTRAHIGH VACUUM OVERVIEW}

\subsection{Vacuum}

To reduce scattering of accelerated particles (electrons and positrons) and photons with residual gas molecules, essentially all major parts of APS are operated under ultrahigh vacuum conditions. ${ }^{1}$ What is vacuum? Although the original Latin word vacuum means "empty," it is impossible to artificially create a space without gas molecules. As shown in Table 1.1, under the hest ultrahigh vacuum conditions achievable at APS there are still millions of residual gas molecules per cubic centimeter, although the gas density has been reduced by ten trillion times. We should bear this microscopic picture in mind when dealing with ultrahigh vacuum problems. The momentum transfer of the perpetual random motion of these molecules gives rise to pressure, which is directly proportional to the number and temperature of gas molecules. Of course these molecules are very tiny. They could travel quite a distance (the so-called mean free path) without hitting another molecule as the pressure drops, as shown in Table 1.1. By the same token our accelerated particles and photons generated from synchrotron radiation will suffer less collision under a good ultrahigh vacuum. In the storage ring for example, under a vacuum better than $1 \times 10^{-9}$ Torr, sufficient beam can survive for more than eight hours without the need of injecting more positrons. Frequent beam injections are very annoying because they interrupt all the experiments on every beam line. It would be much more efficient for APS if we could improve the vacuum further. A better vacuum will also help to maintain a more stable beam intensity, beam size, and beam position. Our goal is to obtain and maintain a vacuum as high as practicable. To understand why it is so difficult to evacuate our vacuum chambers we need some basic concepts about ultrahigh vacuum, especially 
Table 1.1. Gas density and mean free path under various environments for air at $25^{\circ} \mathrm{C}$

\begin{tabular}{|c|c|c|c|}
\hline Environment & $\begin{array}{c}\text { Pressure } \\
\text { (Torr) }\end{array}$ & $\begin{array}{c}\text { Gas Density } \\
\left.\text { (molecules/cm }{ }^{3}\right)\end{array}$ & $\begin{array}{c}\text { Mean Free Path } \\
(\mathrm{cm})\end{array}$ \\
$\begin{array}{c}\text { Normal } \\
\text { Atmosphere }\end{array}$ & 760 & $\sim 2.5 \times 10^{19}$ & $6.7 \times 10^{-6}$ \\
\hline $\begin{array}{c}\text { Linac, Par, } \\
\text { Booster }\end{array}$ & $<5 \times 10^{-7}$ & $<9.0 \times 10^{8}$ & $>2.0 \times 10^{5}$ \\
\hline $\begin{array}{c}\text { Storage Ring } \\
\text { (with beam) }\end{array}$ & $<1 \times 10^{-9}$ & $<3.2 \times 10^{7}$ & $>5.1 \times 10^{6}$ \\
\hline $\begin{array}{c}\text { Storage Ring } \\
\text { (without beam) }\end{array}$ & $<1 \times 10^{-10}$ & $<3.0 \times 10^{6}$ & $>6.0 \times 10^{7}$ \\
\hline $\begin{array}{c}\text { 10,000 miles } \\
\text { above earth }\end{array}$ & $\sim 1 \times 10^{-14}$ & $<50^{-14}$ & $>4.6 \times 10^{11}$ \\
\hline
\end{tabular}

the pumping process and the sources of gas load. We discuss the sources of residual gas first. 


\subsection{Sources of Residual Gases}

There are two kinds of gas sources in a vacuum $s$ stem in addition to the volume gas contained in the system before pumping. One is gases entered into the system from outside through vacuum leaks and the permeation process. The other is internal sources, including surface outgassing from internal surfaces, volume outgassing from the bulk through diffusion, virtual leaks, vaporization of bulk materials, etc. The influence of the permeation process (mainly hydrogen) is more pronounced for pressures lower than $1 \times 10^{-12}$ Torr at elevated temperatures and can be neglected for APS if the right materials are used for vacuum walls and seals. The amount of initial volume gas inside the vacuum system is very small compared to that generated afterwards. The other sources, namely, vacuum leaks, virtual leaks, surface outgassing, volume outgassing, and vaporization are all critical for ultrahigh vacuum at APS and need closer attention. We now discuss them in detail.

Vacuum leaks occur mostly on joints of vacuum parts. One can use permanent seals or demountable seals to join vacuum parts. Welding is widely used for permanent seals and for joining vacuum tubes to flanges. Ultrahigh vacuum welding is considerably different than ordinary welding and should be performed only by trained personnel. Specific welding procedures must be followed. All welding should be done on the inside of chambers with an argon arc to ensure there are no voids which generate virtual leaks. Soldering and brazing are also capable of providing leak-free joints. They should also be done according to ultrahigh vacuum requirements. Demountable seals are specially designed to eliminate leaks through inclusions in the metal components. There are three major metal seals used at APS, namely, the ConFlat flange seal which is most commonly used, the EVAC aluminum knife-edge seal (EVAC International, Inc.) used in the linac tungsten target chamber and in the PAR beamline, and the rectangular knife-edge seal used in the linac waveguide.

The ConFlat flange seal has two symmetric flanges each having an outside rim to confine a flat copper gasket and a concentric knife edge with a right-triangular crosssection. One side of the knife edge is vertical to the flange plane and the second side is inclined at $\sim 70^{\circ}$, as shown in Fig. 1.1a. When two flanges are bolted together with a flat copper gasket in between, the knife edges bite into the gasket causing a lateral flow of copper. The outside rim restricts the flow and forces the copper gasket to seal imperfections in the interface and substantially reduces the size of leak-path channels. This matured technique, developed by Varian over 30 years ago, can provide virtually leak-tight seals better than $10^{-12}$ Torr 1/s. A great deal of caution has been taken to prevent potential leaks. For example, the flanges are made from bar stock instead of plate stock to avoid leak paths resulting from the rolling process. Also the material is usually forged to break up the long filamentary inclusions. The copper gasket is made from oxygen-free-high-conductivity (OFHC) copper and hydrogen annealed after cutting. The use of OFHC copper is important because copper oxide will react with hydrogen to form water vapor, which can create voids in the metal when it is heated. These voids will create a porous leaky material. In general, we find that it is a good practice to purchase commercial ultrahigh vacuum chambers and flanges whenever possible as opposed to 
making your own. Special attention should be paid to protect knife edges and copper gaskets from contamination and scratches. Bolts should be tightened in proper sequence to ensure an even pressure on flanges (as described later in this section). The joint should not bear uneven stress and tension. This is especially important for the other two metal seals used at APS, i.e., the EVAC aluminum knife-edge seal and the rectangular seal used in the linac.

The EVAC design is similar to ConFlat except that the knife edges are on the aluminum gasket itself and the flange surfaces in contact with the gasket are flat, as shown in Figuie 1.1b. The gasket is made of soft aluminum alloy, which makes it easier to fill in the imperfections on the flange surfaces with less compressing force. This is very useful in applications where rapid interconnection is required. For example, by using an aluminum knife-edge gasket together with the EVAC chain clamps and tapered flanges, one can make rapid replacement of tungsten targets in the linac system.

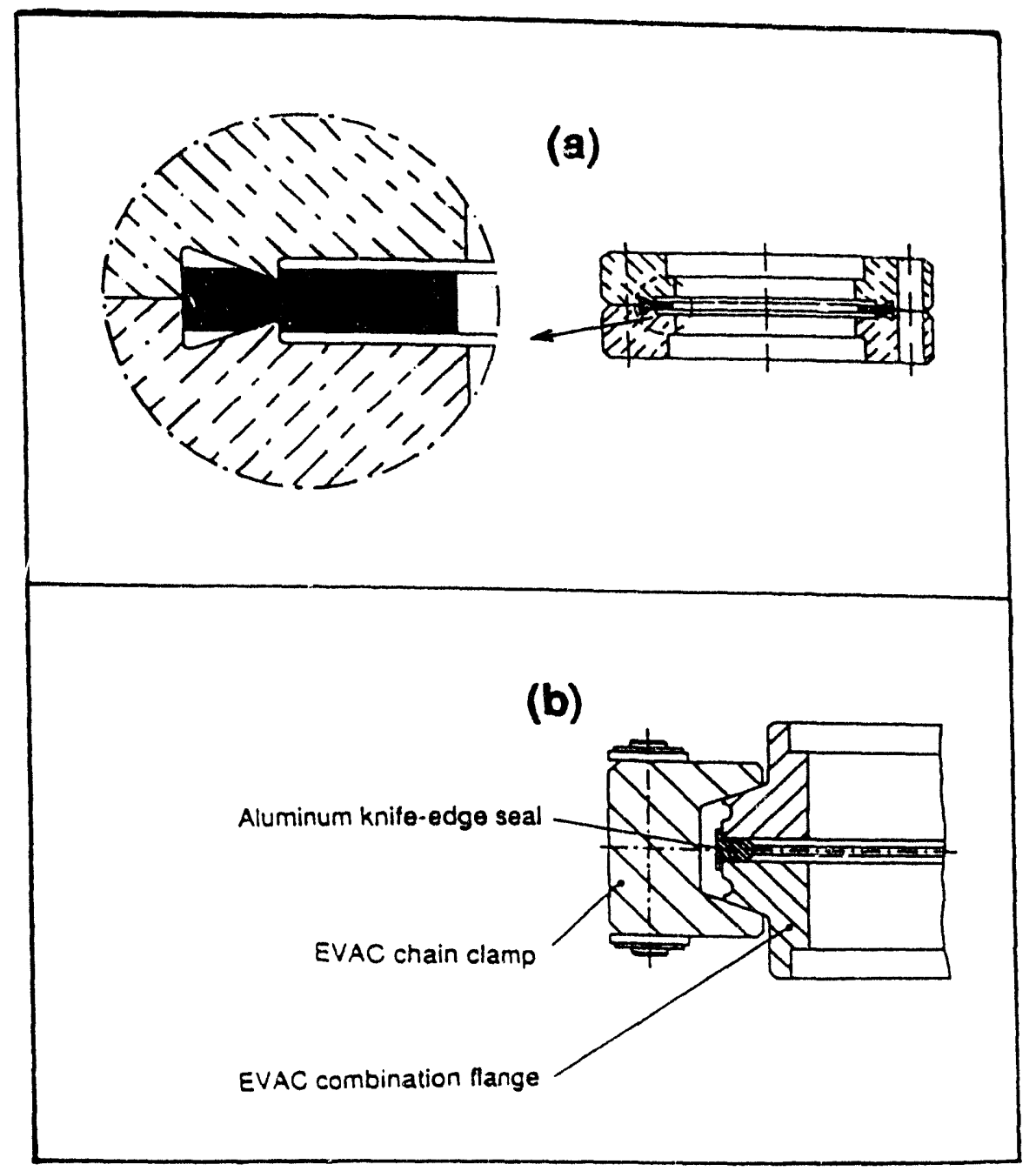

Figure 1.1 (a) Basic design of the ConFlat flange seal from Varian; (b) basic design of EVAC combination flange with a chain clamp and an outer centered aluminum knifeedge seal (EVAC International, Inc.). 
The rectangular type of metal seal for the linac waveguide system is in the process of improvement. The experience we gained from the ConFlat and EVAC seals should be helpful in this respect. The rectangular copper waveguide is brazed to stainless steel flanges with rectangular openings for making connections to other waveguides. These waveguides form long networks to provide if power for the linac. Their rectangular shape and the uneven strain produced by their weight on the joints may present a challenge for the design of vacuum seals. The sealing mechanism of the ConFlat knife should be applied to the design. The level of the knife edge should be lower than that of the flange surface so that the gasket can be well contained in position. The gasket should be made from OFHC copper and annealed in hyd ogen after cutting. Soft aluminum alloy may also be used as gasket material instead of copper for a better seal. When the waveguides are jointed horizontally they should rest on some additional mechanical support to prevent excess uneven stress on the joints.

An important factor which needs attention is the coefficient of linear thermal expansion. For example, at APS there are some joints which are made by a stainless steel - aluminum Conflat flange combination. Aluminum has a higher thermal expansion than stainless steel by a factor of $\sim 1.5$. It will expand more during a bakeout. In this case additional care should be taken when these two flanges are bolted together. The idea is still what we have emphasized before, i.e., to make an even tightening and avoid uneven stress and tension. A useful procedure for bolting flanges is as follows: (1) first tighten the bolts diagonally with a smaller torque (depending on the size of the flange), to make an even close-up; (2) then tighten the bolts clockwise or counterclockwise one by one with a larger torque which is increased gradually after each cycle (or two cycles) until a certain torque is reached. The torque used depends on the size of the flange, the bigger the flange the larger the torque value. For an 8 " flange (stainless steel to stainless steel) the initial torque may be $20 \mathrm{lb}$-in or less, then gradually increased to anywhiere from 26 to $36 \mathrm{lb}$-in. For a stainless steel - aluminum flange combination a much larger torque (up to $70 \mathrm{lb}$-in) may be needed. One should be able to find the best value for oneself according to the above-mentioned principle. For stainless steel - aluminum flange combinations one may also use bolts made from metal materials which have an intermediate value of expansion coefficient. Table 1.2 lists the coefficient of linear expansion for some widely used materials.

Table 1.2 Coefficient of linear expansion $\alpha$ for some widely used materials

\begin{tabular}{|l|c|c|c|c|c|c|c|}
\hline Material & $\mathrm{Al}$ & $\mathrm{Cu}$ & S. Steel & $\mathrm{Be}$ & $\mathrm{W}$ & $\begin{array}{l}\text { (AL15) } \\
\text { Glidcop }\end{array}$ & $\mathrm{Si}$ \\
\hline$\alpha\left(10^{-6 / \mathrm{K})}\right.$ & 23.1 & 16.5 & 15.0 & 11.3 & 4.5 & 16.6 & 4.7 \\
\hline
\end{tabular}

Vacuum leaks through the vacuum vessel may be quite troublesome but they can be easily detected by a leak dicector. In some cases the leak may come from sources inside the vacuum vessel; this kind of leak is called a virtual leak. 
Virtual leaks are more difficult to detect since they are inside the vacuum chamber. A typical example of a virtual-leak source is the trapped air in a blind hole blocked by a screw. When the vacuum chamber is evacuated this trapped air will take a long time to be pumped out because of the very small conductance of the narrow helical crevice. This problem is easily solved by drilling a small venting hole to the trapped area. Virtual leaks can also be found from welds made from the outer surfaces. One should be aware of these problems. Any components to be placed in the vacuum chamber should be properly designed to avoid virtual leaks. Porous materials or materials with voids should also be avoided. These materials will generate more serious surface outgassing.

Surface outgassing is the most critical problem at APS. By definition surface outgassing is the release of gas molecules adsorbed on internal surfaces in the vacuum system. The number of released molecules usually far exceed that in the vacuum space. To understand surface outgassing it will be necessary to discuss how these gas molecules are adsorbed on the surface.

There are physical as well as chemical adsorption processes. In a pure physical adsorption process, gas molecules are held on the surface by the van der Waals forces between the gas molecules and the solid surface atoms. The van der Waals force is very small and a pure physical adsorption usually happens at reduced temperatures. This has been demonstrated in physisorption of inert gases on solid surfaces. For example, in an experiment of physisorbed $\mathrm{Xe}$ on a clean and atomically flat single crystalline palladium substrate we found that there was no Xe adsorption at room temperature. ${ }^{2}$ The Pd substrate had to be cooled to below $50 \mathrm{~K}$ to condense a few atomic Xe layers. As soon as the substrate temperature was raised to $\sim 60 \mathrm{~K}$ all but one close-packed monolayer of Xe were desorbed from the substrate. This experiment tells us that while the binding energy for Xe on Pd is small, it is even smaller for Xe to Xe. Adsorbed Xe atoms always try to find the more favorable sites on Pd surfaces. For gases other than inert gases it is easier to be adsorbed or even absorbed (i.e. to diffuse into the bulk of the surface) on solid surfaces. Moreover, the molecules may dissociate on striking the surface and react with surface particles to form a chemical bond. This chemical adsorption process generally implies electron transfer between the adsorbates and the substrate and involves much higher binding energy than a physical process. In the case of oxygen on aluminum for example, the binding energy is $\sim 1000 \mathrm{~kJ} / \mathrm{mol}$ ( $>10 \mathrm{eV}$ per molecule), which compares to typical values of less than $3 \mathrm{~kJ} / \mathrm{mol}$ for a physical adsorption. Aluminum oxide layers usually have complex structures and may contain up to 100 equivalent monolayers of $\mathrm{CO}$ with binding energies up to $2 \mathrm{eV}$ per molecule. ${ }^{3}$

A special class of gas deserving mention is condensable gases such as water vapors. Water molecules can adsorb on most solid surfaces by many tens of monolayers. The heat of evaporation of water (i. e. the energy needed to make a $100^{\circ} \mathrm{C}$ water into a $100^{\circ} \mathrm{C}$ water vapor) is $2.26 \mathrm{~kJ} / \mathrm{mol}$ or $0.042 \mathrm{eV}$ per molecule. A bakeout procedure is usually applied to desorb water molecules. A $150^{\circ} \mathrm{C}$ bakeout for example, can efficiently desorb most of the adsorbed water (at $150^{\circ} \mathrm{C}$ the thermal energy is about $0.06 \mathrm{eV}$ ). Adsorbed gases with a higher binding energy will require a higher desorption energy and their thermal outgassing rate is low. However, when they are exposed to energetic photon 
and photoelectron bombardment (eV to $\mathrm{keV}$ range) from synchrotron radiation, they will leave the surface rapidly and give a burst in pressure to the system. At the APS storage ring the photon-induced desorption is caused mainly by surface oxide dissociation on electron or photon impact and is the main source of gas load. It is thus very important to reduce the total amount of adsorbed gases.

There are several factors that determine the total amount of adsorbed (and absorbed) gases. One major factor is the surface condition. Both physical and chemical adsorption occur on the surface and the amount of gases adsorbed is proportional to the real microscopic surface area, not the geometric area. More importantly, a porous surface tends to be able to hold multilayers of gases because of the increased area of contact made available to the gas molecules by the pores. In general, one should avoid porous materials and make the surface dense and microscopically smooth. One example is machinable tungsten used at the front end of the APS beamlines. The material will be subject to strong bremsstrahlung radiation (radiation emitted by an electron accelerated in its collision with the nucleus of an atom). It is crucial to reduce the photon-induced desorption from this material. Ordinary tungsten is too hard to be machined. By using powder metallurgy techniques tungsten can be made machinable or made directly into a specified shape. In the process of powder metallurgy, however, enormous voids and grain boundaries may be created. For ordinary use this is not a concern; for ultrahigh vacuum it is another story. A special manufacturing process and heat treatment have to be used to eliminate voids as much as practicable on surfaces of the finished tungsten parts. A significant difference in outgassing between ordinary and specially treated machinable tungsten has been found by the Engineering \& Construction Group of APS. For an ordinary piece $\left(\sim 1 " \times 5^{\prime \prime} \times 8^{\prime \prime}\right)$ put in an ultrahigh vacuum chamber $\left(\sim 2500\right.$ in $\left.^{3}\right)$ the vacuum could reach only $1 \times 10^{-8}$ Torr with a $250^{\circ} \mathrm{C}$ bake and a $400 \mathrm{~V} / \mathrm{s}$ ion pump. For the specially treated piece the vacuum reached $2 \times 10^{-10}$ Torr with only a $150^{\circ} \mathrm{C}$ bake and a 200- $1 / \mathrm{s}$ ion pump. ${ }^{4}$ The importance of surface conditions of UHV parts can not be overstated.

Another factor affecting the amount of adsorbed gases is the cleanliness of the material used in ultrahigh vacuum and how it is cleaned. Components contaminated with grease, oil, fingerprints, etc. will outgas too much for the system to handle and should be thoroughly cleaned before installation. The cleaning procedures are provided in Chapter 3. After cleaning the components usually need to be pre-baked at elevated temperatures to reduce the oxide layer and the hydrogen content in the material and to get rid of most of the adsorbed water. Dry nitrogen is commonly used after the pre-bake and the components are properly sealed before assembling to prevent re-adsorption of water. Care must be taken never to touch UHV components with bare hands. A single fingerprint can ruin the whole pre-bake and cannot be cleaned afterwards in the in situ bakeout after assembling. On a microscopic scale, a single fingerprint is a reservoir for storing gas molecules and it contributes to gas re-absorption into the bulk material.

Under normal conditions adsorbed molecules are released into the vacuum system by thermal agitation. At room temperature this is a very slow process. In situ bakeout is usually necessary to degas the system and thus shorten the time needed to reach an ultimate vacuum. The bakeout process can greatly reduce water content in the vacuum system and hydrogen content in the bulk material but is not that efficient in removing other 
gases. When the system is subjected to synchrotron radiation, more deeply absorbed molecules are released by photon stimulated desorption and give a burst of gas load in the vacuum chamber. The system will clean itself gradually by synchrotron radiation. But the only way one can limit the initial gas load burst and shorten the self-cleaning time is to decrease the total amount of adsorbed and absorbed gases. This can be done by selecting the right materials and cleaning procedures. The bottom line is to ensure a compacted surface and a dense oxide layer as thin as possible. To select the right materials for ultrahigh vacuum use we need to understand two more processes of outgassing, namely, volume outgassing and vaporization.

Volume outgassing is the release of gases through diffusion from the inside of the material to the vacuum-side surface. There is always some gas content inside a material. For example, gases can be dissolved in a metal during the initial melting and casting. Diffusion is a phenomenon of particle movement from the concentrated regions to the dilute regions. The diffusion rate is proportional to the concentration gradient and temperature. It also depends on the mobility of individual diffusion species in the bulk material. In general it is a much slower process than desorption and will contribute to the total outgassing in a later stage of vacuum pump-down when the gas concentration on the surface drops. It is important to select materials with smaller gas contents. It also helps to vacuum-bake the material before assembly.

Vaporization of some material inside the vacuum may also present a vacuum problem. Vaporization is the thermally stimulated entry of molecules into the vapor phase and obviously it depends on temperature. If the kinetic energy of atoms or molecules bound to a solid is sufficient to overcome the binding energy, then the particles will escape into the gas phase. Since synchrotron radiation can deliver a lot of power and cause the temperature of the radiated area to rise substantially, we need to be aware of vaporization problems. A vapor is a gas near its condensation temperature which leaves the surface. In equilibrium, molecules leaving the surface are balanced by those that arrive at the surface. The rate of vaporization depends only on the temperature and nature of the substance; the higher the temperature the higher the equilibrium vapor pressure becomes. The vaporpressure vs. temperature curves for elements and some other materials can be found in most vacuum books. As a rule, the smaller the binding energy of the substance the higher the vapor pressure at a given temperature. Most plastics have high vapor pressure and should not be left in an ultrahigh vacuum system. Some metals, if their atomic subshells are all full, will have a high vapor pressure. Typical examples are zinc and cadmium. Zinc has a full $3 \mathrm{~d}$ shell with 10 electrons and a full $4 \mathrm{~s}$ shell with two electrons on an inner argon core. Cadmium has a full $4 \mathrm{~d}$ shell and a full 5 s shell on a krypton core. The same rule can be applied to mercury, magnesium, calcium, strontium, barium, etc. The physics behind this rule is that the electron transfer between elements with full atomic subshells will be substantially reduced, leading to a smaller binding energy. Other high vapor-pressure materials include sulfur, phosphorous, lead, selenium, etc. Materials containing these elements should be avoided for vacuum applications.

A good understanding of the basic concepts described in this section is very important in material selections for ultrahigh vacuum, which will be discussed next. 


\subsection{Material Selections in Ultrahigh Vacuum}

The materials used for vacuum chambers at the APS are stainless steel, aluminum, and OFHC copper. They all have a low vapor pressure and will not present vacuum problems after proper handling. Each of them will be discussed in the next chapter (APS Vacuum Design). In this section we discuss some general concern for materials and components to be used (or to be avoided) in ultrahigh vacuum.

As mentioned before, to avoid vaporization, components containing zinc, cadmium, lead, sulfur, fluorine, phosphorous, selenium and all high vapor-pressure materials should not be used in ultrahigh vacuum. Examples in this category include brass (which contains zinc), type 303 stainless steel (which has sulfur, phosphorous or selenium), cadmium-plated screws and bolts, common brazing alloys containing zinc and cadmium, tin-lead soft solders, etc.

To reduce the total amount of adsorbed gas it is important to avoid porous materials. The machinable tungsten we mentioned before provides a good example for metals. There are two other metals widely used in photon absorbers at the APS, i.e., Glidcop and beryllium copper. Very high power radiation will impact a photon absorber and generate a great amount of heat. The same precaution to prevent excess outgassing has to be applied to these materials. The commonly used Glidcop at APS contains 0.3 wt.\% aluminum oxide $\left(\mathrm{Al}_{2} \mathrm{O}_{3}\right)$ in a copper host. The aluminum oxide is dispersed into copper for extra mechanical strength, which is needed for the big thermal stress generated on the absorber. Beryllium is a low $\mathrm{Z}$ (atomic number) element and has longer penetration depth for photons. By brazing a beryllium plate onto copper, the absorbed photons can be dispersed into both beryllium and copper, thus dissipating the radiation power in a much larger volume. Nonetheless, substantial heat will still be generated despite the designed water cooling. Although all these materials have a low vapor pressure, one should try to eliminate voids, high vapor pressure impurities, and oxygen contents in the process of manufacturing and brazing these materials. Also, the absorber should be baked in vacuum before assembly at high temperatures.

For non-metals, plastics should be kept away from ultrahigh vacuum. Other nonmetals include ceramics, polymers, glasses, etc.

Ceramics should be carefully examined before assembling. Ceramics are widely used for electrical insulation. They are polycrystalline, non-metallic inorganic materials such as oxides, sulfides, nitrides, borides, carbides, and silicate ceramics, formed under heat treatment. They usually contain entrapped gas pores. Lots of them are too porous to be used in ultrahigh vacuum and are called "bad" ceramics. One should be careful and select "good" ones for UHV use. "Good" ceramics are usually very dense, smooth, and shiny. Quite often they are specifically made for UHV. If you are not sure you should check it out. In general, high density, alumna-based ceramics have much lower gas content than silicate ceramics. They can be further improved by forming the component in vacuum. Some ceramics may contain metal oxide impurities which dissociate at elevated temperatures. It is a good idea to vacuum-bake these components at about $1000^{\circ} \mathrm{C}$ before assembly.

In some situations machinable ceramics (MACOR, for example) can be used in ultrahigh vacuum to provide mechanical support and electrical insulation for some 
components. MACOR is a white porcelain-like material composed of $\sim 55 \%$ fluorophlogopite mica and $45 \%$ borosilicate. It has very little porosity, a quite low outgassing rate when properly baked, and can withstand a relatively high temperature up to $800^{\circ} \mathrm{C}$. Fluorine will "poison" MACOR and should be avoided.

Polymers have found applications in vacuum systems. One application is in elastomers such as Viton seals. They should not be used at APS because of their high permeability and solubility of hydrogen. Polymers are especially vulnerable for vacuum at elevated temperatures and should be avoided completely in such situations. Another application is in polyimide-enameled copper wires. Polyimide has lower outgassing rate than most other synthetic materials after a $300^{\circ} \mathrm{C}$ bake. The polyimide enamel on the wire will not crack when wound around and subjected to heat shock at $400^{\circ} \mathrm{C}$. These wires can be used in ultrahigh vacuum in situations where ceramic insulators are not practical to use, provided the temperature is not too high $\left(<200^{\circ} \mathrm{C}\right)$. One should be aware though, that polyimides adsorb water easily in air and the unbaked degassing rate can be fairly high.

Certain glasses are traditional high-vacuum materials. In modern ultrahigh vacuum systems they are used only to serve a special purpose, e.g., view ports and windows. UHV windows and feedthroughs have glass-metal and ceramic-metal joints. Their thermal expansion coefficients are matched to that of metal alloys. But still one should pay attention to avoid uneven heating of these parts (during a bakeout for example). One should always cover all windows with aluminum foil during bakeout. Windows are usually designed to withstand the pressure from the outside but are vulnerable to a positive pressure from inside the chamber. Care should be taken to prevent pressure build-up in the vacuum chamber when venting the vacuum system.

There are other problems in addition to gas load if unsuitable materials are enclosed in ultrahigh vacuum. For example, if left uncleaned, fluxes used in soldering will produce a huge amount of fluorine. Fluorine will poison the nonevaporable getter (NEG) pumps, which can be a disaster for NEG-pumped vacuum systems. In the next section we discuss how different pumps work. 


\subsection{Pumps and Pumping Processes}

In order to create an uitrahigh vacuum, various vacuum pumps have to be used. There are primarily three kinds of UHV pumps used at APS: NEG pumps, ion pumps, and turbomolecular pumps (backed by roughing pumps). To understand how these pumps work one should bear in mind the nature of constant molecule movement of gases. In ultrahigh vacuum the mean free path for molecular collisions is so long (cf. Table 1.1) that collisions of molecules with solid surfaces (walls, etc.) are more prevalent than collision with other molecules. The gas throughput is dependent upon the geometry of the vacuum system and not upon the absolute density of the gas. This is called the free molecular flow regime. The ultimate pressure is determined not only by the pumping speed but also by the outgassing rate and the geometry of the system. In other words, one cannot improve the vacuum by simply using larger pumps. Pumps can only create traps that collect residual gases. Different pumps have different pumping mechanisms and can be speciesselective for different gases. It is thus important to understand what each type of pump will do and how it will do it.

NEG pumps rely on chemical sorption so that they will only pump chemically active gases. The NEG pumps used at APS are zirconium-vanadium-iron alloy coated on constantan ribbons. These strips can be easily installed in the antechamber of the storage ring to provide distributed pumping, which is crucial for the conductance-limited vacuum chambers (cf. Chapter 2). The $\mathrm{Zr}$ alloy will rcact with oxygen to form oxides, with nitrogen to form nitrides, with $\mathrm{CO}$ and $\mathrm{CO}_{2}$ to form oxides and carbides, and with water to form bulk hydrogen and oxides. Hydrogen is easily absorbed into the bulk of the getter to form a solid solution so that a NEG is very efficient in pumping hydrogen. The absorbed hydrogen can be released upon heating in a later reactivation process. Other compounds formed in the reaction are stable and will be permanently entrapped there.

NEG pumps cannot pump inert gases because of the lack of a chemical reaction. They have very little pumping efficiency for hydrocarbons such as methane $\left(\mathrm{CH}_{4}\right)$ because these gases do not adsorb on the getter surface. Inert gases and hydiocarbons are thus pumped by other means. In the ordinary pumping process, the reactive gases and hydrogen are first adsorbed on the getter surface and then react at the surface and gradually diffuse into the bulk. The surface barrier of formed compounds is shallow enough to permit diffusion but the diffusion process is slow, leading to a slower pumping speed of the pump. When the pumping speed has gradually decreased to a unacceptable level, the NEG can be reactivated by heating to effectively diffuse the surface compounds into the bulk and restore a fresher surface. One can imagine that the whole getter will be eventually exhausted. Even before the complete exhaustion, the accumulated compounds will become so brittle after many thermal cycles that they will peel off from the getter. Usually, under normal operations the progressive reduction of pumping speed due to the accumulation of permanently trapped compounds will end the life of the NEG strips sooner than the thermal fatigue. At APS the NEG will be activated at $450^{\circ} \mathrm{C}$ under a base pressure better than $1 \times 10^{-9}$ Torr and then operated at room temperature. Under these conditions the NEG pump can be exposed to dry nitrogen and regenerated about 40 times and still keep a pumping speed of at least $50 \%$ of its rated value. 
Obviously, the lifetime of NEG pumps depends greatly on how they are used. There are few rules to remember:

(1) The NEG pump is sealed in slightly pressurized dry nitrogen by the manufacturer. Open the seal only immediately before installation.

(2) Never expose NEG to air when it is hot.

(3) Vent the vacuum system only with dry nitrogen.

(4) Operate NEG only in good vacuum (better than $1 \times 10^{-7}$ Torr).

(5) Maximum pressure during activation should never exceed $1 \times 10^{-4}$ Torr.

(6) Avoid "poison" gases.

The NEG pump is very vulnerable if "poison" gases such as fluorine are present. Fluorine is the most electronegative element and the most chemically energetic of the nonmetallic elements. It will react with every component in the getter to form very stable fluorides. When fluorine is present, other gases have little chance to compete for reaction. Moreover, fluorine is able to replace other nonmetallic elements in the existing compounds on the getter surface. The NEG pumps will no longer be able to pump other gases. They cannot be reactivated by heating and have to be replaced. The "poisoned" getter strips may be cleaned by washing them in deionized water since fluorides are soluble in water. 5 One possible source of fluorine comes from the flux used in silver soldering. If silver soldering has to be used, remember to boil the whole soldered part in deionized water to remove the flux. In addition to fluorine, other halides should also be avoided, although they are not as energetic as fluorine.

In addition to nonevaporable getter pumps, other getter pumps such as the titanium sublimation pump (TSP) is widely used. Titanium also reacts with most residual gases. It sublimates quite rapidly at $\sim 1450^{\circ} \mathrm{C}$ so that a fresh $\mathrm{Ti}$ layer can be built onto some internal walls to more efficiently absorb gases. Subsequent sublimation of Ti buries previously absorbed gases permanently. TSP is usually contained inside an ion pump to enhance the pumping efficiency.

Ion pumps can be found in almost every UHV system. In an ion pump gas molecules are ionized in a prepumped vacuum and the resulting ions are forced to move tow: rds the electrodes by a strong electric field. The pump current is a direct measure of the pressure inside the ion pump.

Figure 1.2 demonstrates the pumping mechanism of a typical sputter ion pump. A DC voltage of $\sim 5 \mathrm{kV}$ is applied between a series of ring-shaped anodes and two cathode plates which are made of chemically active metals such as titanium. Gas molecules are ionized when they collide with energetic free electrons. To increase the colliding probability, a pair of permanent magnets are applied along the axes of anode rings so that electrons and ions will move along helical paths. The positive ions are accelerated into the cathodes and eject cathode atoms. These atoms are deposited mainly on the anode surface and act, together with fresh cathode surfaces, as getters. Chemically active gases are thus pumped. For inert gases, they are ionized, and then accelerated toward the cathode and entrapped within several atomic layers there. They may be re-emitted if the entrapping lattice atoms are sputtered away. Typically the pumping speed for inert gases is small. One should avoid heavy use of ion pumps for inert gases; they can be better 
pumped by a turbomolecular pump. In general, to ensure a long lifetime for an ion pump, heavy gas load should always be avoided.

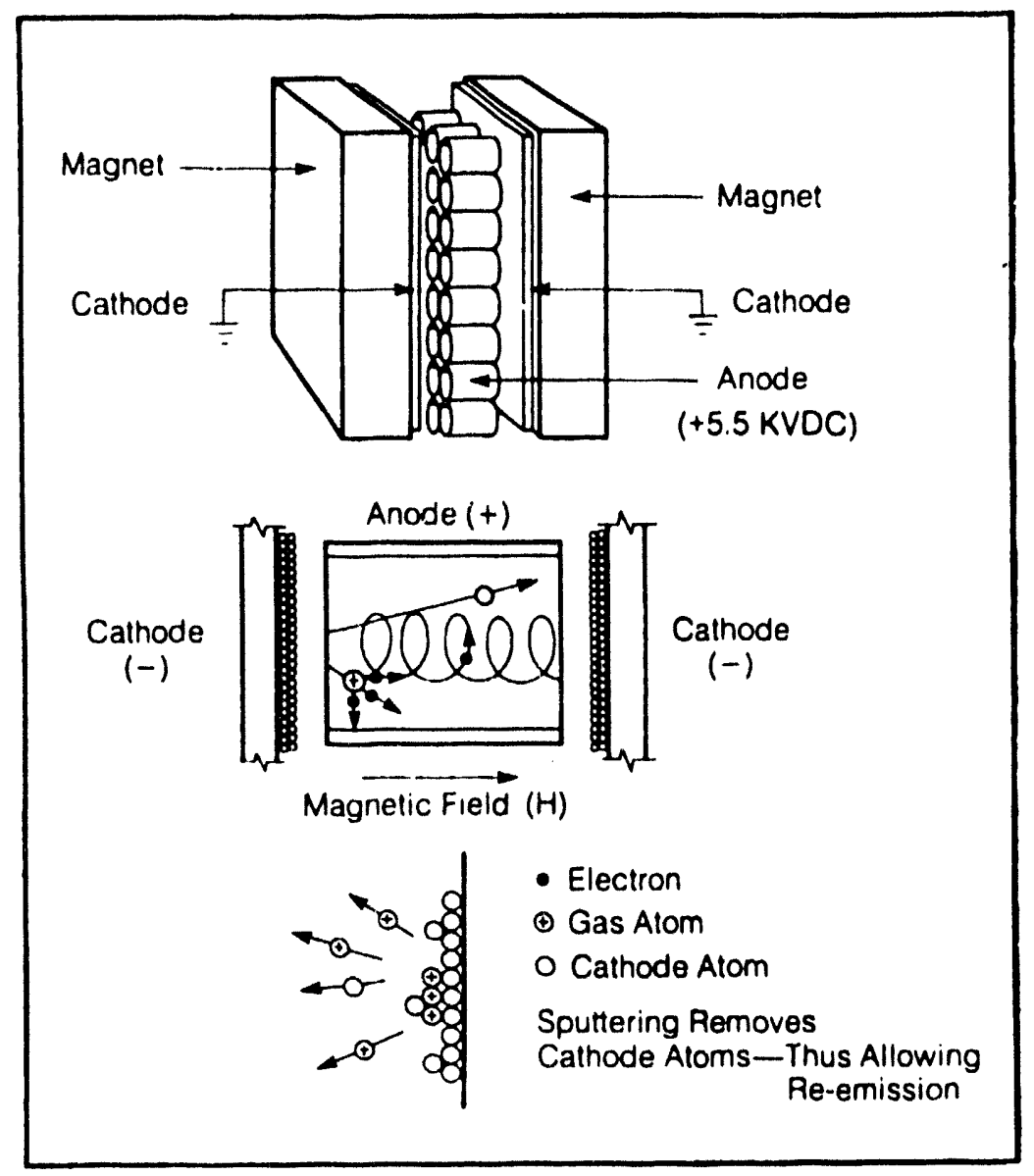

Figure 1.2 Schematic diagrams showing how a sputter ion pump works (reproduced from 1989-1990 Perkin Elmer Ultra High Vacuum Equipment Catalog, p. 29, with the permission of the copyright holder).

Failure of aged ion pumps is commonly caused by the peel-off of brittle deposits. These tiny flakes are attracted to the area between cathode and anode and may create an electrical short. In the early stage when this happens, one may use a high-current power supply connected between anode and ground to burn off the shorting flakes. Usually a couple of burn-offs are necessary to completely eliminate these shorts. Sometimes when a DC power supply is used, a reversal of electrical polarity is helpful to neutralize the static charge on the flakes. Although eventually the electrodes of an ion pump will have to be cleaned or replaced, the simple treatment just described may keep a shorted ion pump working for a few more years. Usually the flakes are the results of ion pump abuse. When the gas load is too high the ion pump can get very hot. Repeated excess heat makes the deposit layer brittle and it will eventually peel off. Prolonged continuous 
operation of an ion pump above $1 \times 10^{-4}$ Torr should be avoided. When the pressure is higher than $\sim 1 \times 10^{-3}$ Torr, a discharge will occur if the ion pump is left on. A starting pressure of $\sim 5 \times 10^{-4}$ Torr or better is recommended. Turbopumps are usually used to obtain the required starting pressure.

Turbomolecular pumps are specially designed mechanical type pumps based on the molecular drag principle, i.e., when a gas molecule hits a rapidly moving surface it will be dragged along and thrown in the direction of rotation with an extra velocity. A turbopump consists of specially shaped rotor and stator blades with slots set at an angle so that gas molecules caught in the slots of the moving plates will be projected in the direction of the slots on the stator plates. The rotational speed of the pump is so high (>tens of thousands rpm) that the circumferential velocity is comparable to or larger than that of gas molecules. The appropriate design then ensures the gas molecules are pushed into the fore vacuum side and pumped away by a fore pump. With the help of the fore pump the turbopump works in the molecular flow region where the gap between rotor and stator plates $(\sim 1 \mathrm{~mm})$ is smaller than the mean free path of the gas, resulting in a higher probability of a molecule going out to the fore vacuum region. Turbopumps are very efficient in pumping heavy molecules but are much poorer for hydrogen. A good turbopump can pump a vacuum system from atmosphere pressure down to UHV range. It would be advantageous though if the system at atmosphere could be pumped first by sorption pumps.

Sorption pumps are simply cylinders containing activated molecular sieves. Vacuum is generated when they are cooled to liquid nitrogen temperature. When the valve separating the sorption pump and the vacuum system is opened, the gases in the system, including hydrogen, will be rapidly sucked into the sorption pump until an equilibrium is reached. This kind of initial pumping can greatly reduce the workload of a turbopump. The sorption pump can be easily regenerated by heating.

An alternate to the sorption pump is the dry mechanical pump. Oil-sealed mechanical pumps are not allowed to directly pump a UHV system because of the back streaming of hydrocarbons (these pumps may be used as roughing pumps for the turbopump).

Due to the very high speed of the rotating blades, any foreign matter such as a piece of broken wire or ceramics entering the pump will be a disaster to the turbopump. Screens at the UHV end of the pumps may be used to prevent foreign matter from entering the pump at the cost of a $15 \%$ reduction in pumping speed. UHV pumps are all quite expensive. Common sense based on good knowledge of ultrahigh vacuum can be very valuable for APS. 


\subsection{Common Sense in Ultrahigh Vacuum Related Work}

This section summarizes some general rules which we should bear in mind.

First of all, people make things happen at APS. Like any other big project, the success of APS depends on the effort of every single person involved. Here is Rule Set \#1:

Whatever you do, please do not blow up APS.

Never leave anything that does not belong to the system inside the vacuum chamber.

Never touch vacuum parts and internal surfaces with bare hands.

Follow specified procedures.

To have a good vacuum, we need on the one hand to limit the gas load and on the other hand to increase pumping efficiency. Rule Set $\$ 2$ concerns reducing the gas load:

Select low-outgassing and low-vapor pressure materials with smooth and dense surfaces.

A void thick and porous oxide layers on the vacuum surface.

Eliminate all detectable leaks.

Eliminate contamination from all sources.

Vacuum is created by using various vacuum pumps. We should keep our pumps at their best working conditions at all times. Rule Set \#3 is about ensuring the pumping efficiency:

Never abuse or overload vacuum pumps - instructions should be followed.

Protect pumps from "poisoning," corrosion, and damage.

Use only oil-free pumps for ultrahigh vacuum.

Another instance of common sense is personal safety, which deserves a separate section to emphasize its importance. 


\subsection{Vacuum Safety Issues}

As in any other work, safety issues should be kept in mind in vacuum-related work. Potential hazards include electrical hazards, hazardous materials, radiation, stored energy, etc. Below we give some examples of electrical hazards.

The danger of electrical shocks is always present whenever electricity is used. This is especially true when high voltage is involved. In the vacuum systems at APS, high voltage sources are present almost everywhere, e.g., rf power sources, ion pumps, ionization gauges, etc. The main potential danger comes from improper grounding of the vacuum chamber. If this happens, lethal voltages can be built up on the vacuum chamber through gas discharges in a poor vacuum. Therefore, it is very important that all vacuum chambers be properly grounded to support at least 10 amperes of current.

Another example is the danger of worn heat tapes during a vacuum bakeout. If the insulation of the heat tape is broken, the exposed wire may not only present an electrical shock hazard but may also cause a short through the vacuum chamber or a bellow. The electrical short may burn a hole on the wall of the chamber or bellow and cause a vacuum failure. It is important to keep all heating tapes in good working condition and discard broken ones. Proper fuses or circuit breakers should also be used during the bakeout.

Various safety rules have been developed to make our workplace a safer one. Common sense combined with adherence to rules and procedures will help everyone prevent unwanted occurrences.

APS has been designed to deploy combined vacuum pumps to ensure a good vacuum for satisfactory operation. In the following chapter we present the design considerations and results of vacuum calculations. 


\section{APS VACUUM DESIGN}

\subsection{Introduction}

Some knowledge of vacuum design will help us to understand the vacuum system better. Moreover, by comparing the pressure distributions calculated from the design parameters with that actually measured, we can diagnose problems in the vacuum system. Once we understand how vacuum systems work it becomes much easier to understand the APS vacuum design.

Vacuum design involves the geometric dimensions, surface outgassing, and effective pumping speed of the system. The dimensions are determined according to other physical requirements of the APS project, with the vacuum concern in mind. In the calculations presented in this chapter we take the geometric dimensions from the actual APS design as known parameters and calculate the pressure distribution of the vacuum system. The outgassing rate is proportional to the surface area and depends on the type and condition of the material. It is determined experimentally and can be found in the literature. However, in the literature one may find different outgassing rate values for the same material. Since it is known that the outgassing rate (for both thermal desorption and photon-stimulated desorption) depends on the surface conditions, one should use a value which is obtained from experiments under similar conditions to one's own. The practical unit for the rate of thermal outgassing is "Torr liter per second per $\mathrm{cm}^{2}$." The total thermal outgassing is simply a multiplication of thermal outgassing rate by the total surface area. The gas load due to the photon-stimulated desorption is also expressed as "Torr liter per second." Pumping speed is in units of "liter per second." As a rough estimation, the ultimate pressure of a vacuum system is obtained by simply dividing the total gas load by the total effective pumping speed. Vacuum calculations are discussed in more detail below.

There are two general considerations in a vacuum calculation. First of all, the molecular flow of gases is limited by the geometry of the vacuum ducts. This consideration is represented by the concept of conductance. The second consideration is the dynamical aspect of outgassing and the pumping process.

Conductance between two places in a vacuum system represents the ease with which gases can flow from one place to the other. In the free-molecular-flow regime (which is the case for ultrahigh vacuum), the conductance is independent of pressure and is determined by the geometry of the system, the temperature, and the mass of the gas under consideration. A pressure difference will be built up because of the limited conductance for the gas flow. The pressure buildup is proportional to the gas throughput and inversely proportional to the conductance between the two places. The gas throughput (defined as the product of the pumping speed and the inlet pressure) is in "Torr liter per second," so that the conductance is conveniently expressed in "liter per second," the same unit used for the pumping speed. One can imagine that if there are several paths connecting the same two places, the resulting conductance would be the sum of the conductance for each individual path. If the gas flow has to go through several paths in series, the net conductance will then be smaller than that of each path. An often- 
used formula for this situation is expressed as the reciprocal of the sum of individual reciprocals. The effective pumping speed $S$ for a pump of pumping speed $S_{p}$ connected to a vacuum system through a duct of conductance $C$ can also be calculated in this manner, i.e., $S^{-1}=S_{p}^{-1}+C^{-1}$.

There are two ways to calculate the conductance of a duct. One is to use standard formulas, which have been worked out for certain simple geometries. For complex shapes, Monte Carlo simulations are used to calculate the probability of gas molecules transmitting through the system. The conductance is equal to the product of transmitting probability and the entrance aperture-conductance. According to Maxwell-Boltzmann statistics, the aperture conductance $C_{A}$ is determined as,

$$
\mathrm{C}_{\mathrm{A}}=3.64 \cdot \mathrm{A} \cdot(\mathrm{T} / \mathrm{M})^{0.5} \text { liter } / \mathrm{sec} \text {, }
$$

where $A$ is the area of the aperture, $T$ the temperature, and $M$ the molecular weight. For air at room temperature, $C_{A}=11.7$ - A liter/sec. In our calculations we assume the residual gas composition is air for the linac and PAR. For the booster ring, the storage ring, and insertion devices, a composition of $75 \%$ bydrogen and $25 \% \mathrm{CO}$ is assumed. In the latter case, the $C_{A}$ is larger than that of air by a factor of 3.4.

For a duct of length $L$ and with a uniform cross section of perimeter $\mathrm{H}$, a commonly used formula of conductance is

$$
\mathrm{C}=[1+(3 \cdot \mathrm{L} \cdot \mathrm{H}) /(16 \cdot \mathrm{A})]^{-1} \cdot \mathrm{C}_{\mathrm{A}}
$$

In a Monte Carlo simulation, a model representing the structure of a duct is first built in the computer. Next molecules are generated from the entrance facet in the model using a random number generator and then bounced off the walls according to some assumed rules until they either leave the duct or return to the entrance facet. By comparing how many molecules have hit the exit facet to the total number generated, one obtains the probability factor of transmission.

The actual vacuum system of APS (or even part of APS) consists of a lot of vacuum parts and is too complicated to be simulated all at once. In practice, the pressure distribution is calculated for each major part of APS using finite element analysis. Each system is divided into a number of elements and its pressure distribution is obtained by solving a matrix equation. The conductance, volume, thermal outgassing, photonstimulated outgassing, and pumping speed for each element are calculated individually and entered into the computer for matrix calculations. The influence of the pressure difference upon neighboring elements is included in the throughput as a product of the conductance and the pressure difference. The respective conductances between neighboring elements is calculated from the individual conductances using the formula of conductances in series. The following equation forms the base of the matrix equation, 6,7

$$
Q_{i}+C_{i-1} \cdot\left(P_{i-1}-P_{i}\right)+C_{i} \cdot\left(P_{i+1}-P_{i}\right)=P_{i} \cdot S_{i}+V_{i} \cdot d P_{i} / d t
$$


where $Q_{i}$ is the total gas load in element $i$, including both thermal and photon-stimulated desorption, $C_{i}$ is the conductance between element $i$ and element $i+1, S_{i}$ is the pumping speed, $V_{i}$ is the volume, and $P_{i}$ is the pressure of element $i$. By applying this equation to all the elements and using some algebra one can construct a matrix equation which can be solved for $\mathrm{P}_{\mathrm{i}}$ by using a computer.

The photon-stimulated desorption $\mathrm{Q}_{\mathrm{PD}}$ usually slows down upon prolonged radiation. This self-cleaning process is represented by the expression of

$$
\mathrm{Q}_{\mathrm{iPD}}(\mathrm{t})=\mathrm{Q}_{\mathrm{iOPD}} / \mathrm{t}^{0.63} \text {, }
$$

where $t$ is the radiation time. $Q_{i P D}$ depends on the amount of synchrotron radiation and the material of each element. The crotch absorber for example, will get the most radiation and thus the biggest desorption among all elements. The straight sections will usually get less radiation than bent sections, leading to a smaller $Q_{i P D}$ in straight sections. In our calculation, we first determine the total amount of $\mathrm{Q}_{\mathrm{PD}}$ for the system. $\mathrm{Q}_{\mathrm{PD}}$ depends on the number of photons generated, $\mathrm{N}$, and the desorption yield per photon, $\eta$. In a synchrotron, $\mathrm{N}$ is directly proportional to the energy of the beam, and the beam current. The desorption yield per photon depends on the material. The storage ring for example, will have $\sim 1.82 \times 10^{21}$ photons per second at $7 \mathrm{GeV}$ and $300 \mathrm{~mA}$ operation. 1 The booster ring, with much less bearn current of $\sim 4.8 \mathrm{~mA}$ and $3.5 \mathrm{GeV}$ on average, will have $\sim 1.46 \times 10^{19}$ photons per second. The desorption yield for stainless steel and aluminum are determined experimentally and can be found in the literature. The total amount of desorption is calculated according to: 1

$$
\mathrm{Q}_{\mathrm{PD}}=6.22 \times 10^{-20} \cdot \mathrm{N} \cdot \eta \text { Torr } \mathrm{l} / \mathrm{s} \text {. }
$$

It is then distributed to each element according to the distribution of radiation power.

The thermal desorption $\mathrm{Q}_{\mathrm{TD}}$ is small and can be considered as time independent in those parts where $\mathrm{Q}_{\mathrm{PD}}$ dominates, such as in the storage ring. However, in parts such as the linac, there is no synchrotron radiation and the vacuum chambers are usually not baked. In this case $\mathrm{Q}_{\mathrm{TD}}$ dominates and decreases upon pumping according to

$$
\mathrm{Q}_{\mathrm{TD}}(\mathrm{t})=\mathrm{Q}_{\mathrm{OTD}} / \mathrm{t}^{\mathrm{n}},
$$

where $\mathrm{n}$ varies from 1 to 1.3 for most unbaked metals.

In the following sections we present results of finite element analysis for major parts of the APS. The computer programs used are modified from an existing program 7 to reflect the difference among different sections. A typical one can be found in the appendix. 


\subsection{Linac Accelerator Chamber}

APS beam begins as electrons emitted from an electron gun which are accelerated to $200 \mathrm{MeV}$ in the electron linac vacuum chamber. Positrons are generated in the positron converter chamber (which contains a tungsten target for the accelerated electrons) and accelerated to $450 \mathrm{MeV}$ in the positron linac vacuum chamber before being injected into the PAR (positron accumulator ring). The accelerating power for the linac is provided by a rectangular feed waveguide system, which is separated from the beamline accelerator chamber by if windows. This will be discussed in the next section.

The accelerator chamber is specially designed so that if power can be efficiently applied. This requirement influences the shape of the chamber, the so-called disc-loaded accelerating waveguide, as shown in Fig. 2.1. There are 14 chambers like this in the linac: five for the electron accelerator and nine for the positron accelerator. The major concern for the vacuum of the accelerator chamber is whether the limited conductance will lead to too high a pressure in the center of the chamber. Vacuum calculations are thus required.

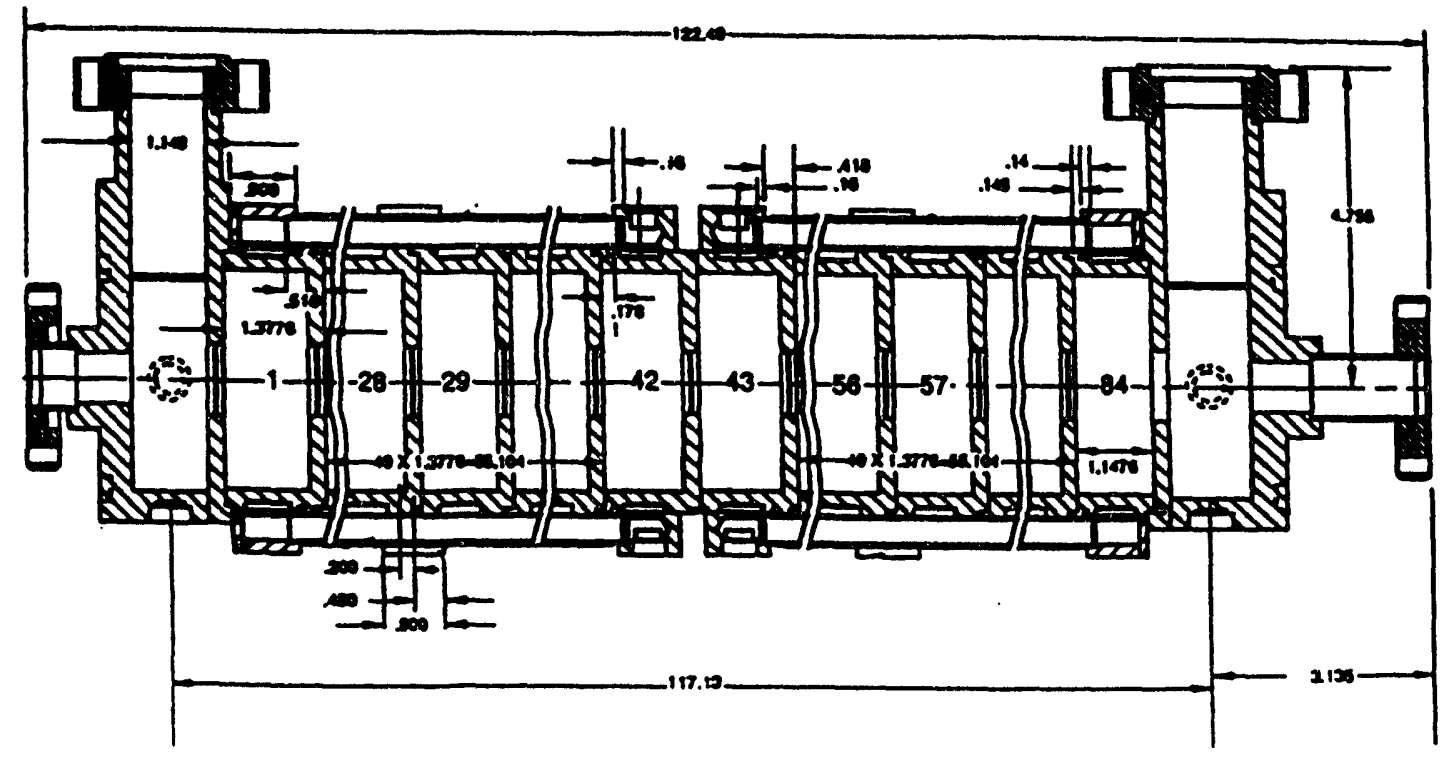

Figure 2.1 A schematic diagram of the linac accelerator disc-loaded waveguide. Numbers $1-84$ indicate 84 cells. 
The chamber consists of 86 cells made from OFHC copper, including two end couplers, with the iris diameter varying smoothly from $2.622 \mathrm{~cm}$ to $1.924 \mathrm{~cm}$. Figure 2.1 shows the cross profile of the chamber. To calculate the conductance of these cells, a Monte Carlo MOLFLOW program ${ }^{8}$ is applied. Figure 2.2 demonstrates a Monte Carlo simulation process of two cells in series. By using mirror reflections, only the top half of the two cells needs to be simulated. Figure $2.2 \mathrm{a}$ is a plot of the top half of two cells and Fig. $2.2 \mathrm{~b}$ records the random paths of the generated molecules.

One may also use the analytical formula and Haefer's addition theorem ${ }^{9}$ to calculate the conductance. In this case, each cell is a series of two cylindrical tubes with two different radii. The difference between the analytically calculated result for two cells in series and that obtained from Monte Carlo simulations is within 7\%. The rounded shape of the disc edge in each cell (cf. Fig. 2.1) is ignored in both calculations. The actual conductance may thus be larger than that calculated.

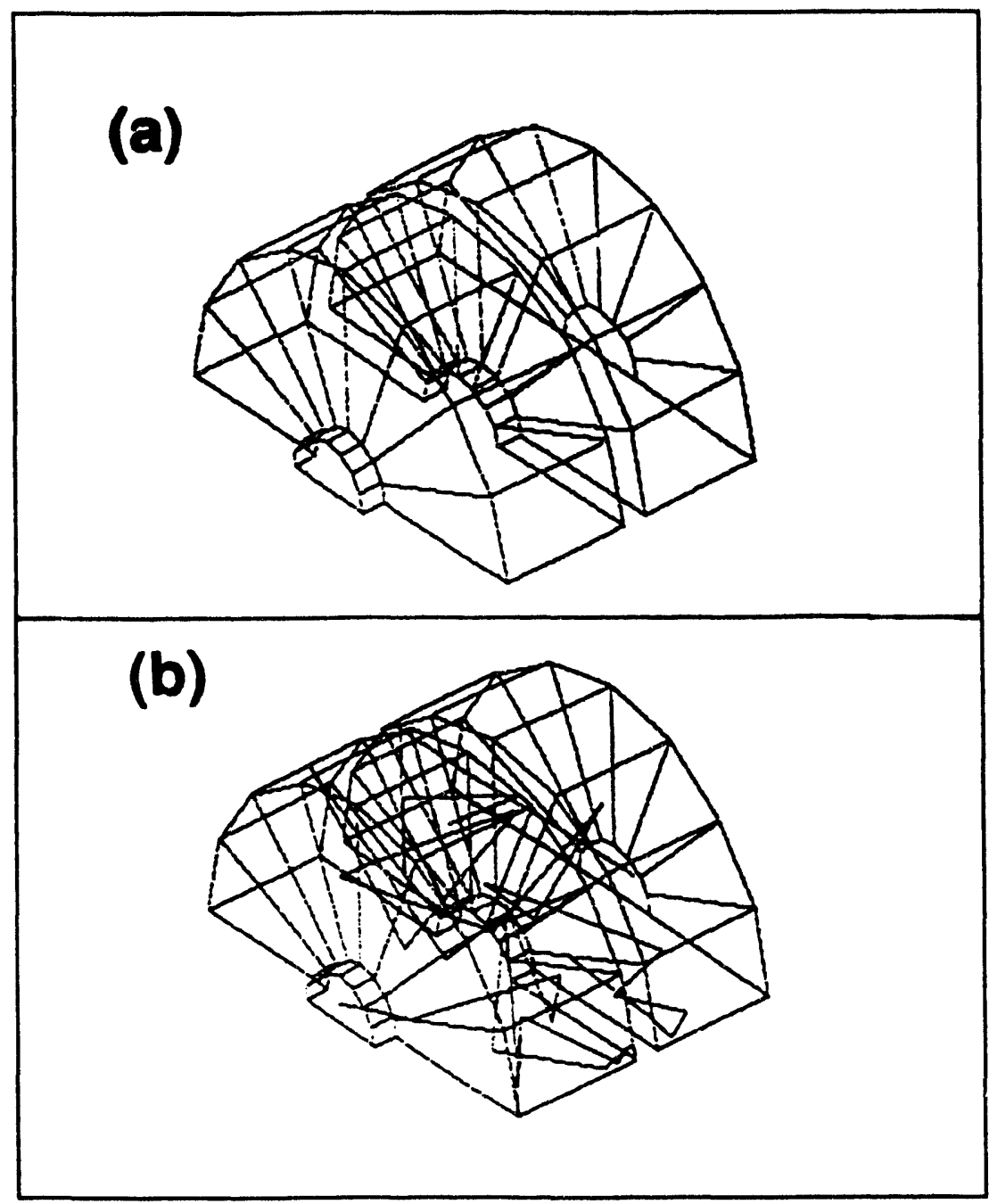

Figure 2.2 (a) A model of the top half of two linac cavity cells used in Monte Carlo calculations. (b) A demonstration of random paths of the generated molecules in a Monte Carlo simulation. 
The chamber is pumped by two $16-1 / \mathrm{s}$ ion pumps connected to the end cells, each through a standard 2.75" 4-way cross and a 21.38 " long tube. (In a final assembly the tube may be shortened. We stay with the long tube so that the calculation is on the conservative side.) The effective pumping speed on the inlet of the chamber becomes $6.65 \mathrm{~V} / \mathrm{s}$ because of the limited conductance of the long tube. The limited conductance of the chamber will build up a higher pressure at the center cells than at the end cells. A pressure of $5 \times 10^{-7}$ Torr at the center cell is required to prevent rf electrical breakdown and to reduce particle scattering.

The main gas load comes from the thermal desorption of OFHC copper surfaces of the chamber. Considering that there will be no in-situ bake after assembly of the chamber, we use $q(t)=q_{0} t^{-1.3}$ with $q_{0}=2.5 \times 10^{-8}$ Torr $1 / s$ per $\mathrm{cm}^{2}$ to calculate the thermal desorption, where $t$ is in hours. The pressure in the chamber will thus improve upon pumping. The abnve empirical expression is valid for $t$ in its first few tens of hours.

Figure 2.3 shows the pressure distribution along the linac vacuum chamber after 10 hours and 48 hours of pumping, obtained by using the finite element analysis program. The calculation shows that if everything goes well, the design objective of a pressure of $5 \times 10^{-7}$ Torr at the center of the linac accelerator chamber can be achieved in about two days. The pressure at the end cells will be $5 \times 10^{-8}$ Torr. By then, one should read a pressure at the ion pump lower than $5 \times 10^{-8}$ Torr.

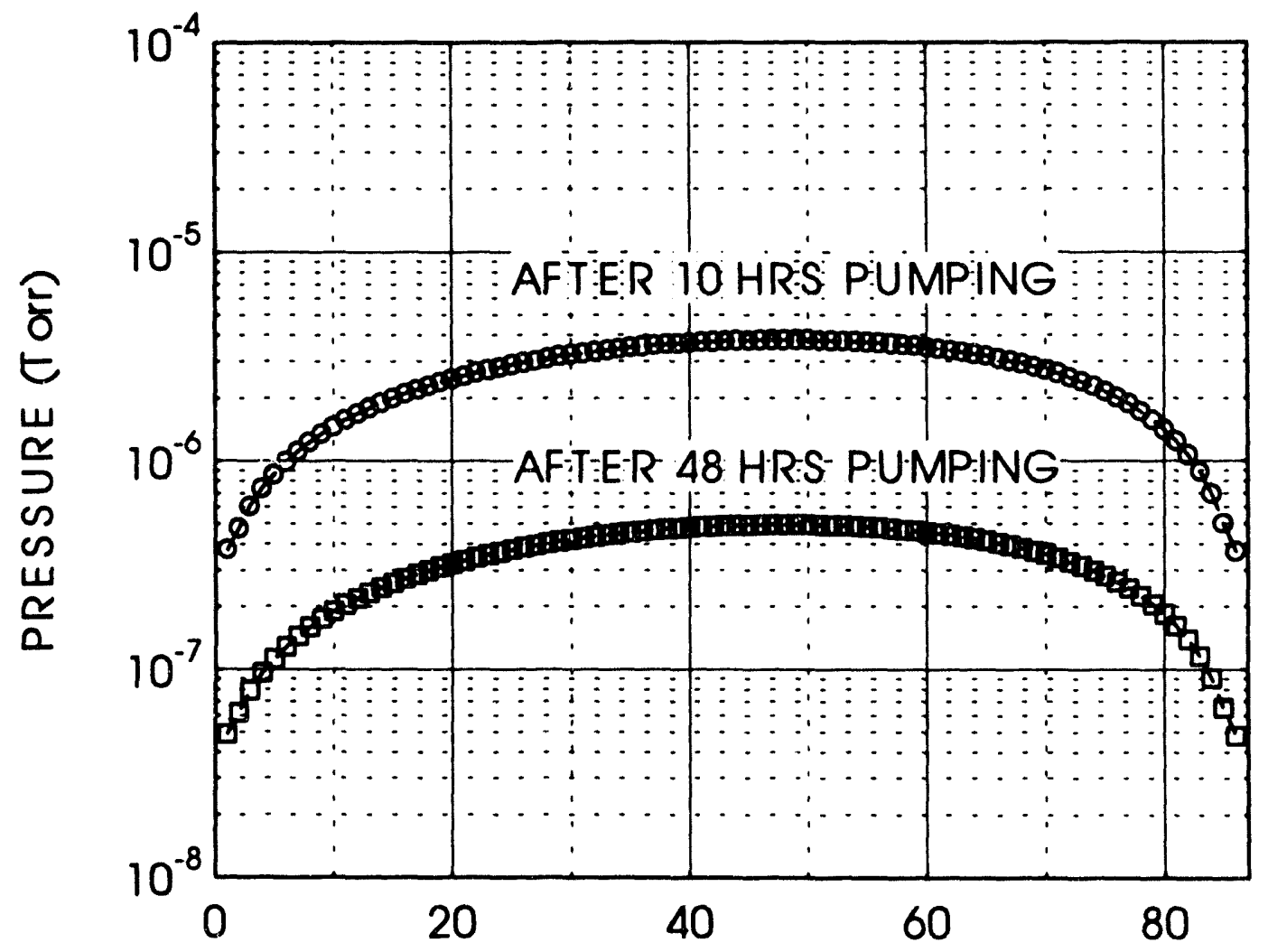

\section{ELEMENTS}

Figure 2.3 Pressure distribution along a linac accelerator chamber after 10 hours and 48 hours of ion pump pumping. 
The calculation is conservative considering that the round edges of the cavity iris are simplified as rectangular edges in the conductance calculations. The vacuum will continue to improve during the operation, but at a slower pace. In other words, it will take even longer than 420 hours to reach an outgassing rate of $1 \times 10^{-11}$ Torr $1 / \mathrm{s} \mathrm{per} \mathrm{cm}^{2}$. The outgassing rate of $1 \times 10^{-11}$ Torr $1 / \mathrm{s}$ per $\mathrm{cm}^{2}$ was widely used in previous calculations for OFHC copper. We feel that the dynamical calculation, such as the one presented in Fig. 2.3, is more practical for an unbaked system. The good news is that according to our conservative calculation, the vacuum pumping for the linac accelerator chamber under present design should be adequate. 


\subsection{Linac Rectangular Waveguide System}

The rf power generated by klystrons are fed into the accelerating structures through rectangular waveguide modulator assemblies. There are three klystron modulator assemblies using the SLAC-SLED system ${ }^{10}$ to power the accelerating sections of the electron and positron linacs and two additional assemblies without SLED to power the electron injector and the first accelerating section of the positron linac. ${ }^{1}$ These five klystrons are isolated by if windows which are transparent to of in the output waveguide. This allows each klystron to be independently evacuated and operated. The rectangular waveguide system is evacuated to prevent electrical breakdown which may occur due to the high-voltage gradients in the waveguide. The vacuum requirement is $\sim 10^{-7}$ Torr at full if power and $\sim 10^{-8}$ Torr at zero of power. Since klystron modulator assemblies are isolated from each other, we present vacuum calculations for only one of them, namely, the assembly number 2 , or Sector 2 . Figure 2.4 shows a schematic drawing of major branches of the Sector 2 waveguide system. Some branches are omitted from the drawing because of the symmetry.

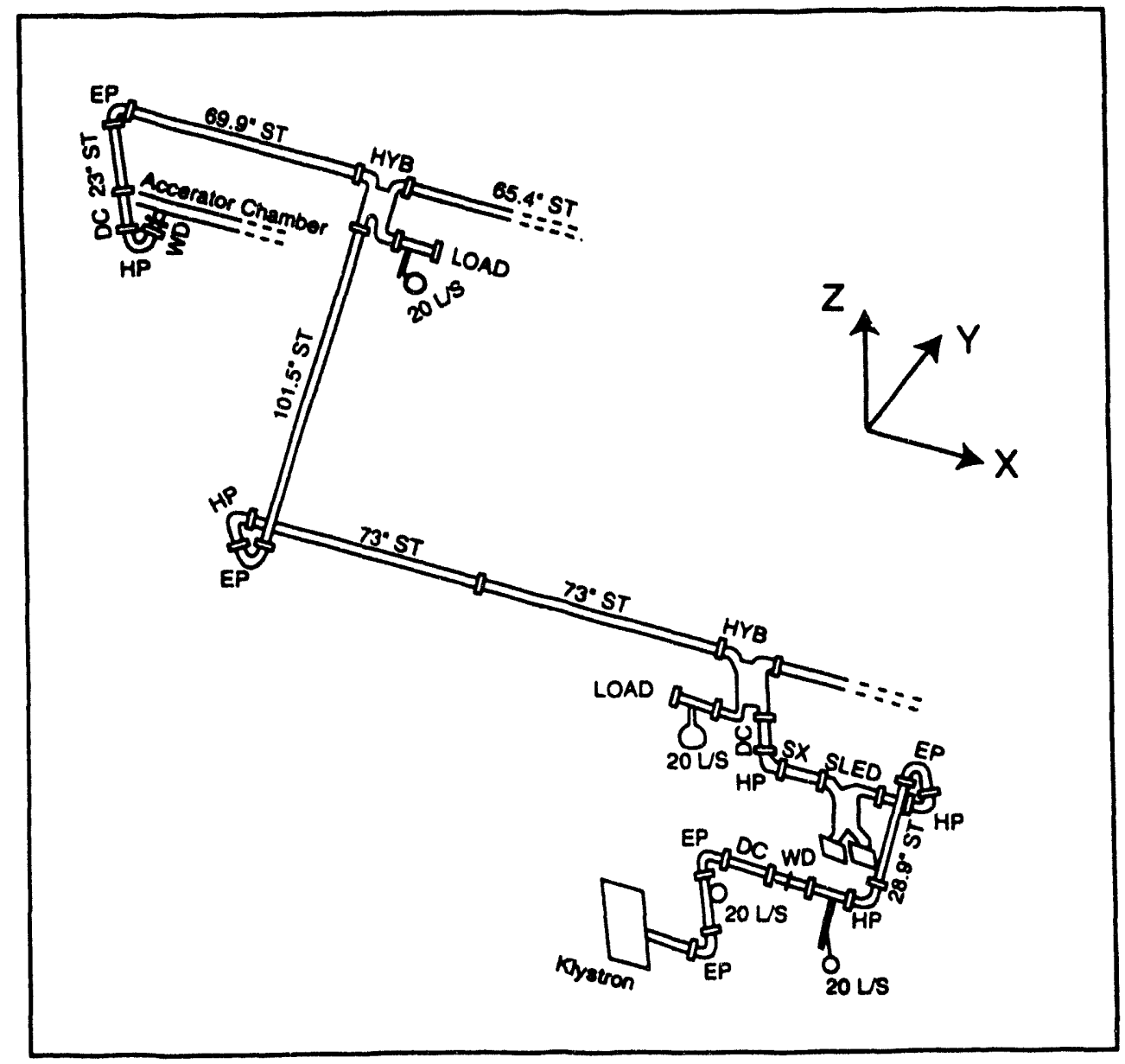

Figure 2.4 A schematic drawing of the Sector 2 rectangular waveguide network. The dashed lines represent the symmetric branches which are omitted in the drawing. 
The Sector 2 waveguide system provides microwave power to the electron linac accelerator with the use of SLED. The SLED microwave network utilizes a dual cavity which is tuned to resonance. ${ }^{10}$ The waveguide assemblies are made of OFHC copper and connected to each other using rectangular stainless steel flanges. The cross section of the waveguide is $1.34^{\prime \prime} \times 2.84^{\prime \prime}$. HP and EP $90^{\circ}$ bends are used to change the direction of a waveguide. Figure 2.5 shows a drawing of an HP $90^{\circ}$ waveguide bend. Monte Carlo simulations are used to calculate the conductance of these complicated structures.

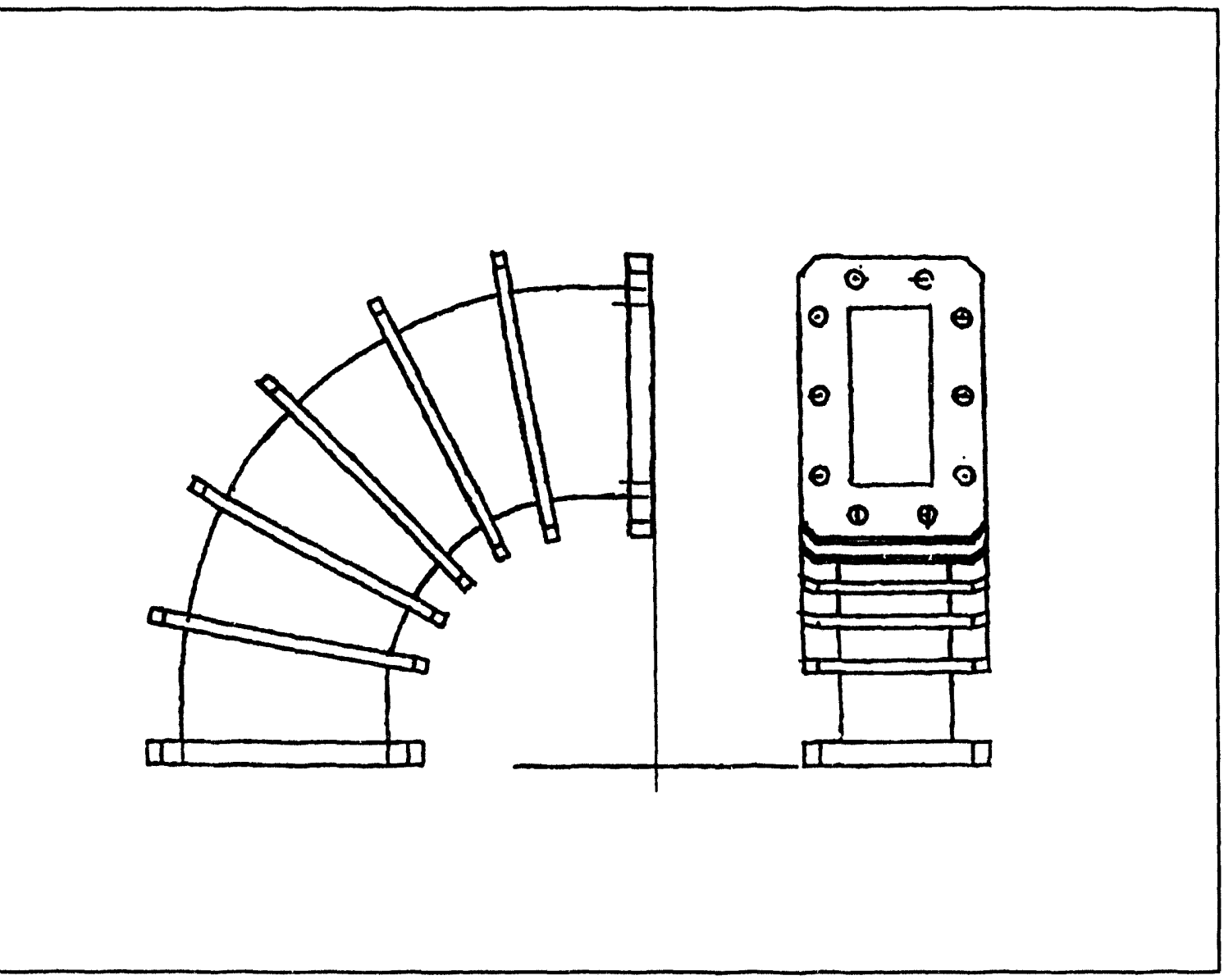

Figure 2.5 A drawing of the HP $90^{\circ}$ waveguide bend.

The waveguide network (cf. Fig. 2.4) is pumped by four 20-1/s ion pumps. There is another ion pump close to the klystron. Since this part is separated from the SLED part by a window, it is not included in the calculation. The ion pumps are connected to the waveguide through T-pipes with an inner diameter of 1.37". The conductance of the Tpipe limits the effective pumping speed to only $10.5 \mathrm{~V} / \mathrm{s}$.

There is no in situ bakeout for the waveguide so we use $\mathrm{q}=2.5 \times 10^{-8} \mathrm{t}^{-1.3}$ (Torr $1 / \mathrm{s}$ per $\mathrm{cm}^{2}$ ) to calculate the thermal outgassing as a function of pumping time, as we described before. The same finite element analysis computer program is used to calculate 
the pressure distributions along the waveguide network. To simplify the calculation, the network is divided into two parts, namely, the one connected to the linac accelerator chamber with three HYB hybrid cavities and the one connected by the SLED (cf. Fig. 2.4). Some assumptions are made to take into account the influence of gas load from those branches which are connected to the system but are ignored in the calculation. The calculated pressures at the joint of these two parts are compared to make sure that this method is justified.

Figure 2.6 shows the pressure distribution along the waveguide network connecting to the linac accelerator. There are four branches connected to the linac accelerator but only the two end ones are included in the calculation. The SLED branch connected to the center HYB is also not included. The gas load of the onitted branches is added to the connecting HYB (with a limited conductance in mind). The pumps are connected to the HYBs so that the pressure at those places is lower than that at other elements. The elements at the far end have the highest pressure due to the limited conductance.

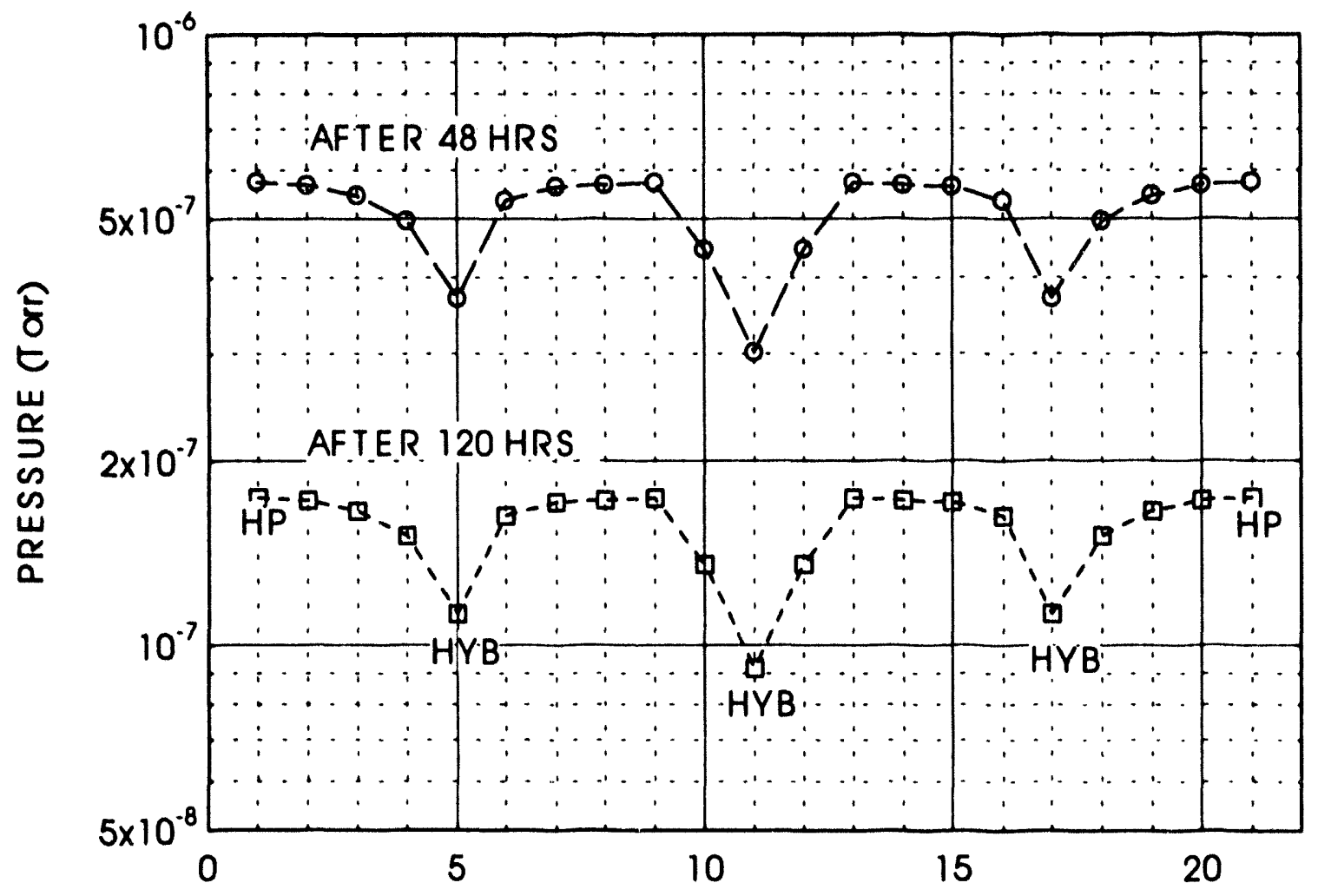

Figure 2.6 Pressure distribution along the Sector 2 rectangular waveguide network. (refer to Figure 2.4 for locations of the elements.) 
The SLED branch is calculated separately. The calculated pressure distribution is shown in Figure 2.7. The pumping for the branch is through pump out (PO). The element DC is connected to HYB which is also pumped by another ion pump. As a result, the pressure at PO and DC are lower than that at other elements. From Figure 2.6 and Figure 2.7 we can see that the SLED branch will not have a significant influence on the pressure of the main waveguide network.

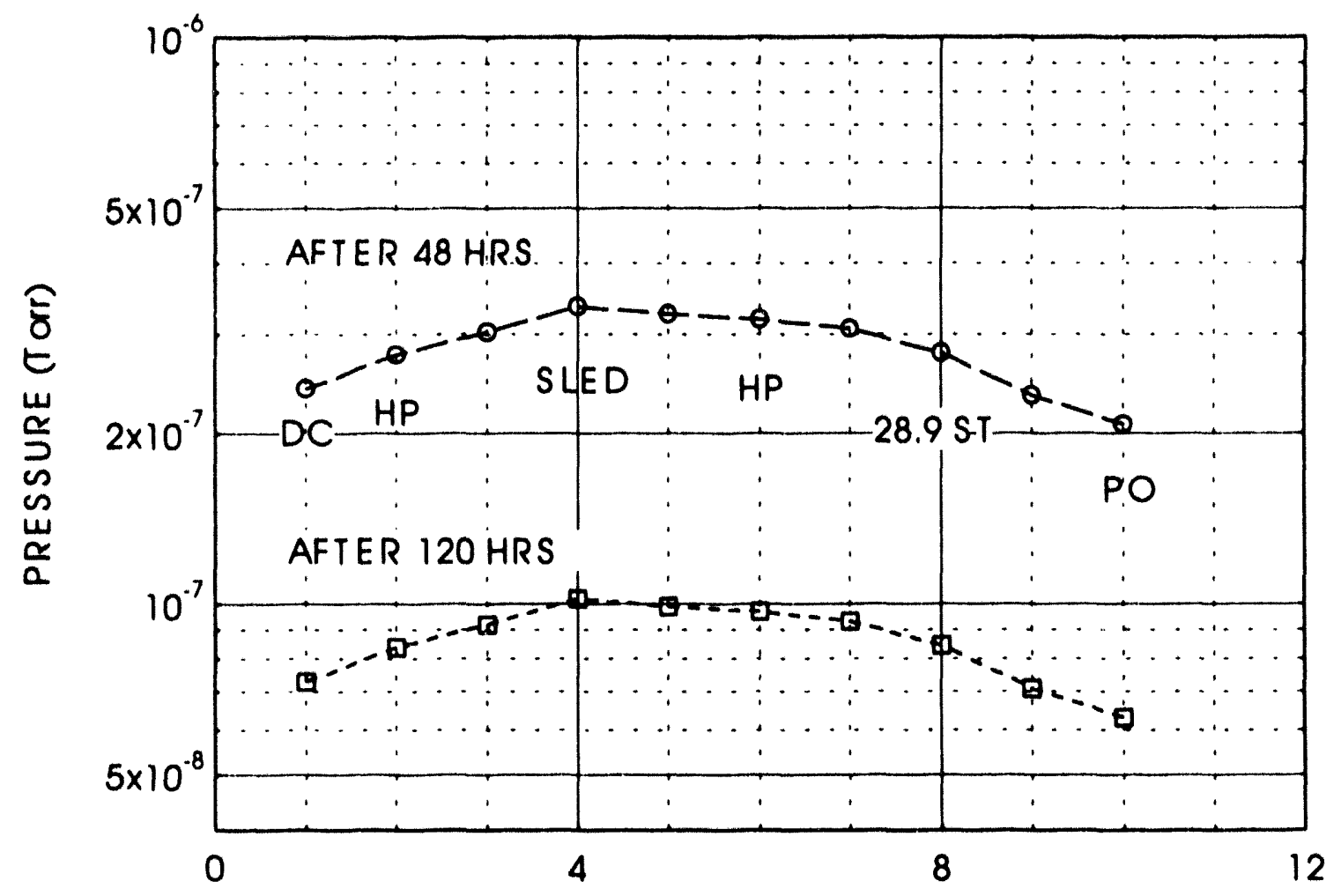

ELEMENTS

Figure 2.7 Pressure distribution along the SLED branch of the Sector 2 waveguide network. (Refer to Figure 2.4 for locations of the elements.)

To monitor the pressure at the waveguide network, one can use the ion pump readings. To correlate the ion pump readings to pressures at the different elements of the network, we have calculated the pressure profile of one branch, from the HP bend through HYB and the T-pipe, etc. to the ion pump. Figure 2.8 shows the pressure profile. We can see from the figure that the ion pump reading is roughly a factor of 4-5 lower than the actual pressure in the waveguide, due to the limited conductance. 


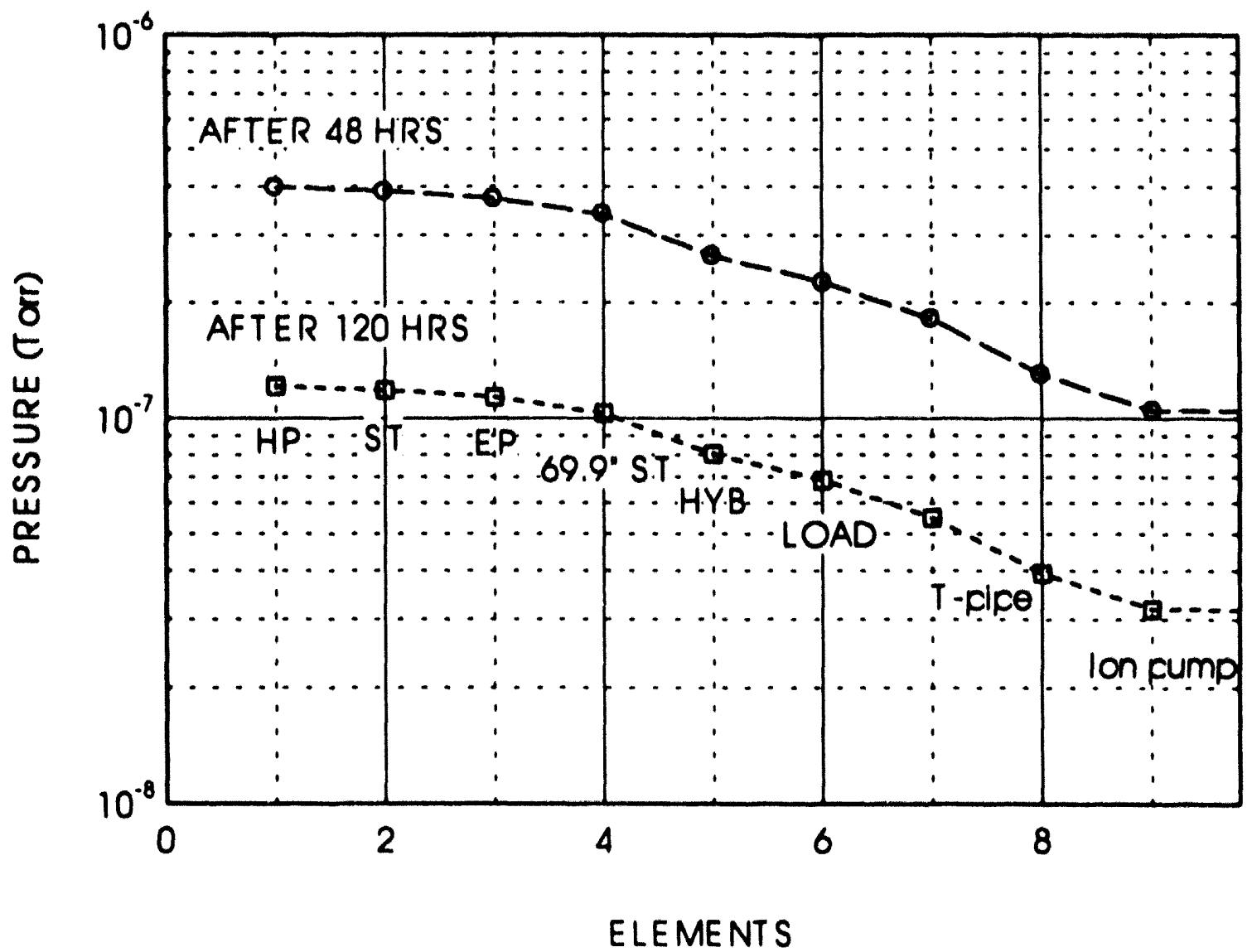

Figure 2.8 The pressure distribution from the waveguide to the ion pump. (Refer to Figure 2.4 for locations of the elements.)

Our calculation indicates that the present vacuum design for the rectangular waveguide network is a little shy of reaching the desired vacuum of $1 \times 10^{-8}$ Torr (without If power). To improve the vacuum, we suggest increasing the conductance of the T-pipe which connects the ion pump to the waveguide. If the effective pumping speed can be increased from the present $10.5 \mathrm{Vs}$ to $16 \mathrm{Vs}$, the average pressure in the waveguide can reach as low as $10^{-8}$ Torr in five days. Since the present design can only marginally meet the vacuum requirement (assuming an average handling in the process of assembly and no bakeout), special care as outlined in Chapter 1 for ultrahigh vacuum should be applied. The waveguides should be prebaked. Detectable vacuum leaks should not be present. The vacuum will continue to improve during operation as long as there are no detectable vacuum leaks. 


\subsection{Low Energy Undulator Test Line}

An undulator test beamline utilizing a very low emittance electron beam from the linac was developed in 1993. This beamline will be used to test the effect of the magnetic field errors within the undulator and the undulator's ability to force the beam to generate high quality light. During the operation of this beamline there will be no tungsten target in the linac; consequently a 700-MeV electron beam will come from the end of the linac. The undulator test line branches off the second PTB (PAR to Booster) bend and enters the undulator test area located in the linac/injection wing. Figure 2.9 shows the layout of the test line.

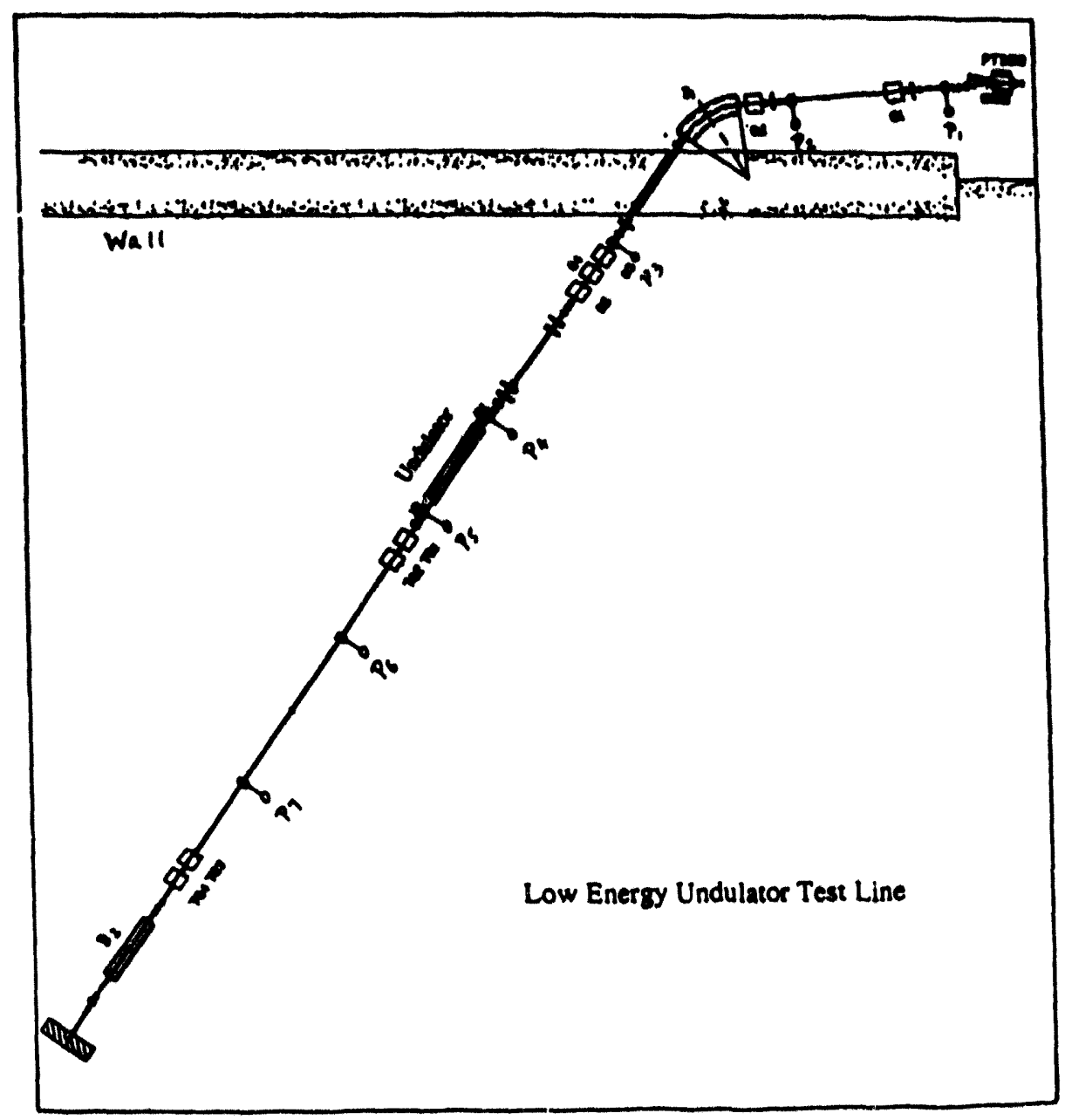

Figure 2.9 Layout of the low energy undulator test line, where $P_{1}$ through $P_{7}$ indicate ion pumps. 
The magnet lattice of the test line consists of two dipoles, nine quadrupoles, and seven corrector magnets. The vacuum chamber of this test line is made of simple $\phi 2 "$ stainless steel pipes. The vacuum requirement is $1 \times 10^{-8}$ Torr for the beamline to minimize bremsstrahlung radiation. There are two $30-1 / \mathrm{s}$ ion pumps connected to both sides of the undulator through two housing chambers where the effective pumping speed becomes $28 \mathrm{~V} / \mathrm{s}$. There are an additional five 30-1/s ion pumps distributed along the beamline through standard $\phi 2.5 " T$ connectors. The effective pumping speed at the $T$ connector is $22.8 \mathrm{~V} / \mathrm{s}$ as calculated.

There is no radiation on the vacuum walls of the test beamline so that the photonstimulated desorption is zero. For a prebaked stainless steel vacuum chamber with an option of in situ bakeout, we can safely assume that the thermal outgassing rate is better than $1 \times 10^{-11}$ Torr $1 / \mathrm{s}$ per $\mathrm{cm}^{2}$.

The finite-element analysis of the low energy undulator line design (cf. Fig. 2.9) indicates that an average pressure of $\sim 6 \times 10^{-9}$ Torr can be obtained when seven $30-1 / \mathrm{s}$ ion pumps are used. Furthermore, our calculation indicates that we can use five ion pumps instead of seven to achieve a $1 \times 10^{-8}$ Torr average pressure. Figure 2.10 shows the pressure distribution along the test beamline for cases of five and seven ion pumps.

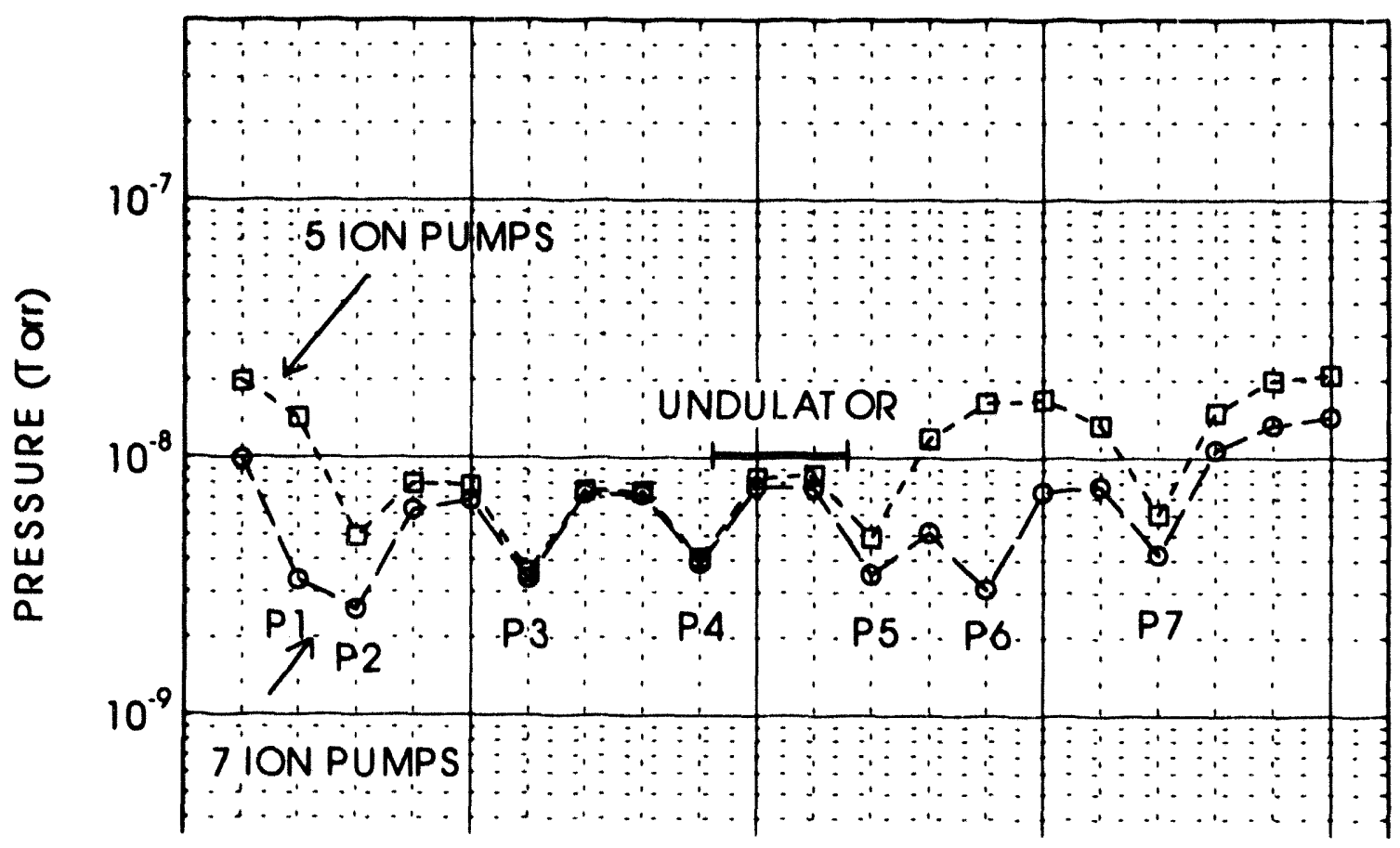

Figure 2.10 Pressure distribution along the low energy undulator test line. The top curve shows that the vacuum requirement for the test line can still be satisfied when pumps $P_{1}$ and $P_{6}$ are removed. 


\subsection{Positron Accumulator Ring}

When APS is in normal operation the 450-MeV positrons from the positron linac are injected into the positron accumulator ring (PAR) through the low energy transport line. A second transport line connects PAR to the booster ring. Figure 2.11 shows the relative position of PAR to the positron linac and the booster. The vacuum design for the low energy transport line is similar to that of the low energy undulator test line and will be omitted here.

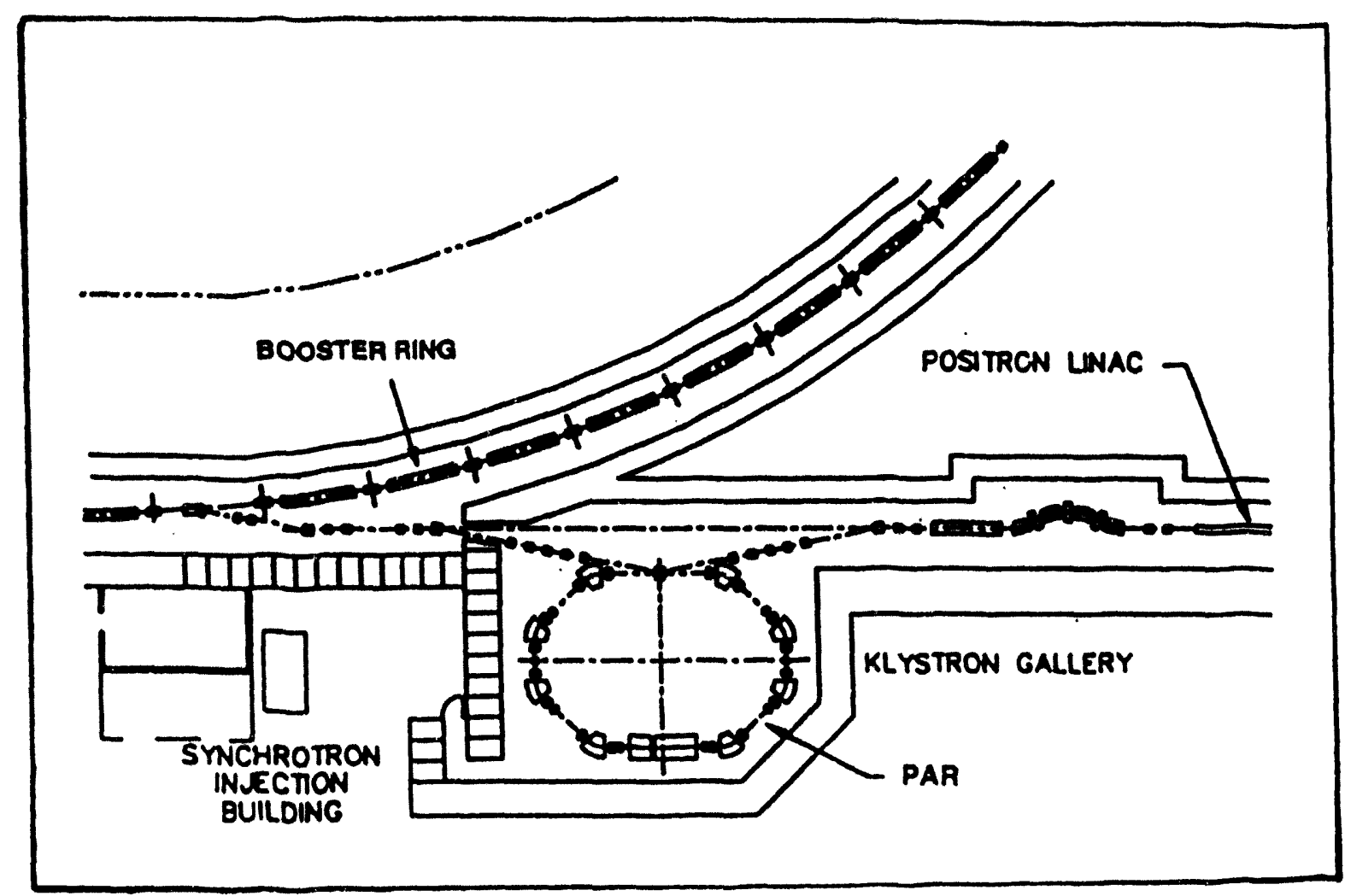

Figure 2.11 Layout of the PAR to the positron linac and the booster ring.

PAR is used to speed up the filling rate of the storage ring. As many as 24 linac pulses can be accumulated in PAR every half second as a single bunch which is then dumped into the booster ring.

Figure 2.12 shows the layout of PAR. It is a $\sim 30.6-\mathrm{m} \mathrm{DC}$ storage ring containing 1 pulsed septum magnet, 3 extraction kickers, 8 bending magnets (bend radius $\sim 1.02 \mathrm{~m}$ ), 16 quadrupoles, 10 multipoles (each contains three independent sets of coils for sextupole and correction dipoles), some ports for vacuum pumps and fluorescence screens, and the 
If section. 11 The vacuum chambers are essentially rectangular tubes made of Inconel (a $\mathrm{Ni}-\mathrm{Cr}$ - $\mathrm{Fe}$ alloy with $55 \% \mathrm{Ni}$ ). Inconel has a high electrical resistivity which is needed to reduce eddy current losses in changing magnetic fields. The inner cross section of the vacuum chamber is $3.8 \mathrm{~cm} \times 12.2 \mathrm{~cm}$. There are a total of 12 ion pumps $(60 \mathrm{l} / \mathrm{s} \mathrm{each})$ distributed along PAR (the additional four ion pumps close to the septum magnet and the If section are not shown in Fig. 2.12). The pumps are connected to the vacuum chamber through a $\sim 30$ "-long $\phi 4$ " pipe, which limits the pumping speed to $\sim 42 \mathrm{~V} / \mathrm{s}$. There are also If screens on the vacuum-chamber opening, which further reduce the effective pumping speed of each ion pump to $\sim 37 \mathrm{l} / \mathrm{s}$. The vacuum requirement for PAR is better than $1 \mathrm{x}$ $10^{-8}$ Torr.

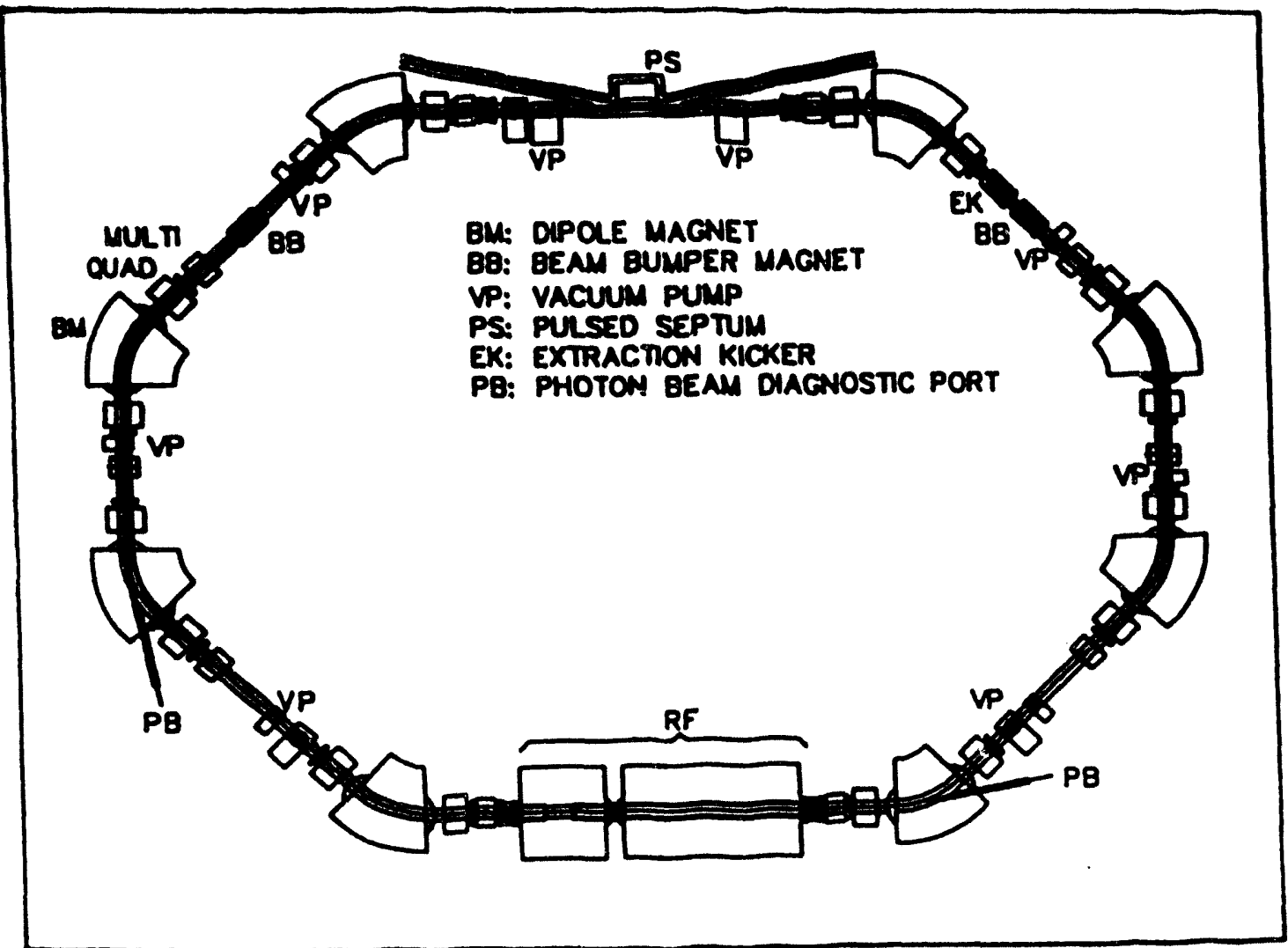

Figure 2.12 Layout of the positron accumulator ring.

We may need to consider the photon-stimulated desorption in our vacuum calculations for PAR since there are circulating positrons. There are 24 linac pulses 
accumulated in PAR every half second. Each pulse contains $0.25 \mathrm{nC}$ or $1.5 \times 10^{9}$ positrons. These positrons circulate in PAR at a period of $102 \mathrm{~ns}$ per turn, leading to an average current of $\sim 34 \mathrm{~mA}$ (assuming a $60 \%$ accumulation efficiency). The relatively small positron energy of $450 \mathrm{MeV}$ limits the number of produced photons to $\sim 1.2 \times 10^{18}$ per second, with a critical energy of $\sim 41 \mathrm{eV}$. Photons with small critical energy will usually give a smaller desorption yield $\eta$. No experimental data for $\eta$ exists for either Inconel or for $41-\mathrm{eV}$ critical energy photons. Foerster et al. 12 have carried out detailed studies on photodesorption from copper, beryllium, and stainless steel using photons with critical energy of $500 \mathrm{eV}$. We may use their data to estimate the effect of photodesorption for our systems.

To consider the possible photodesorption in PAR, we take Foerster et al.'s data for stainless steel in the beam-dose range from $10 \mathrm{~mA} \cdot \mathrm{hr}$ to $4 \mathrm{~A} \cdot \mathrm{hr}$. Their data in that range can be presented as a linear function in the logarithm scale. Or

$$
\eta=\log ^{-1}(-2.7447-0.4184 \log x)
$$

where $\mathrm{x}$ is the beam dose in $\mathrm{mA} \cdot \mathrm{hr}$. The total photodesorption is then

$$
Q_{P D}=6.22 \times 10^{-20} * N * \eta=7.5 \times 10^{-2} * \eta
$$

$\mathrm{Q}_{\mathrm{PD}}$ is distributed along the PAR. We use a weighted distribution, namely, assuming that the straight sections are subjected to only $10 \%$ of radiation and the eight bending-magnet sections bear the most radiation.

PAR is divided into 46 elements for the finite element analysis. The division of the elements and the physical dimensions and surface areas of each element are derived from the related APS drawings. Vacuum chambers contained in the bending magnets, the kickers, and the if section are identified as individual elements. The septum magnet chamber is divided into two elements (No. 1 and No. 46). Some considerations about the vacuum calculation for different elements are discussed below.

The thermal outgassing rate for Inconel is taken as $1 \times 10^{-11}$ Torr $1 / \mathrm{s}$ per $\mathrm{cm}^{2}$, assuming a good bakeout and handling. Some elements such as the kickers and if cavities contain ceramic parts. The septum magnet is inside the vacuum chamber so the detailed chamber shape and a larger outgassing rate have to be considered. The rf section contains a $100.8 "$ "-long, $\phi 6 "$ stainless steel pipe and two 4"-long, $\phi 6.25 "$ ceramic-coated tubes. The thermal outgassing rate for these elements is assumed to be $1 \times 10^{-10}$ Torr $1 / \mathrm{s}$ per $\mathrm{cm}^{2}$.

In our calculation we assume that the thermal outgassing rates are fixed and the photodesorption rates decrease upon photon radiation from the accumulated positrons, as we described before. Figure 2.13 shows the pressure distribution along PAR after it has been subjected to $34 \mathrm{~mA} \cdot \mathrm{hr}, 68 \mathrm{~mA} \cdot \mathrm{hr}$, and $4 \mathrm{~A} \cdot \mathrm{hr}$ of the beam dose (or after 1 hour, 2 hours, and 120 hours under an average positron current of $34 \mathrm{~mA}$ ). From this figure one can see that after an initial burst in pressure (mainly in the bending-magnet chambers), a good vacuum can be obtained after two hours of operation. Further improvement of the vacuum is expected because of the self-cleaning effect of both the photon and the if stimulated desorptions. 


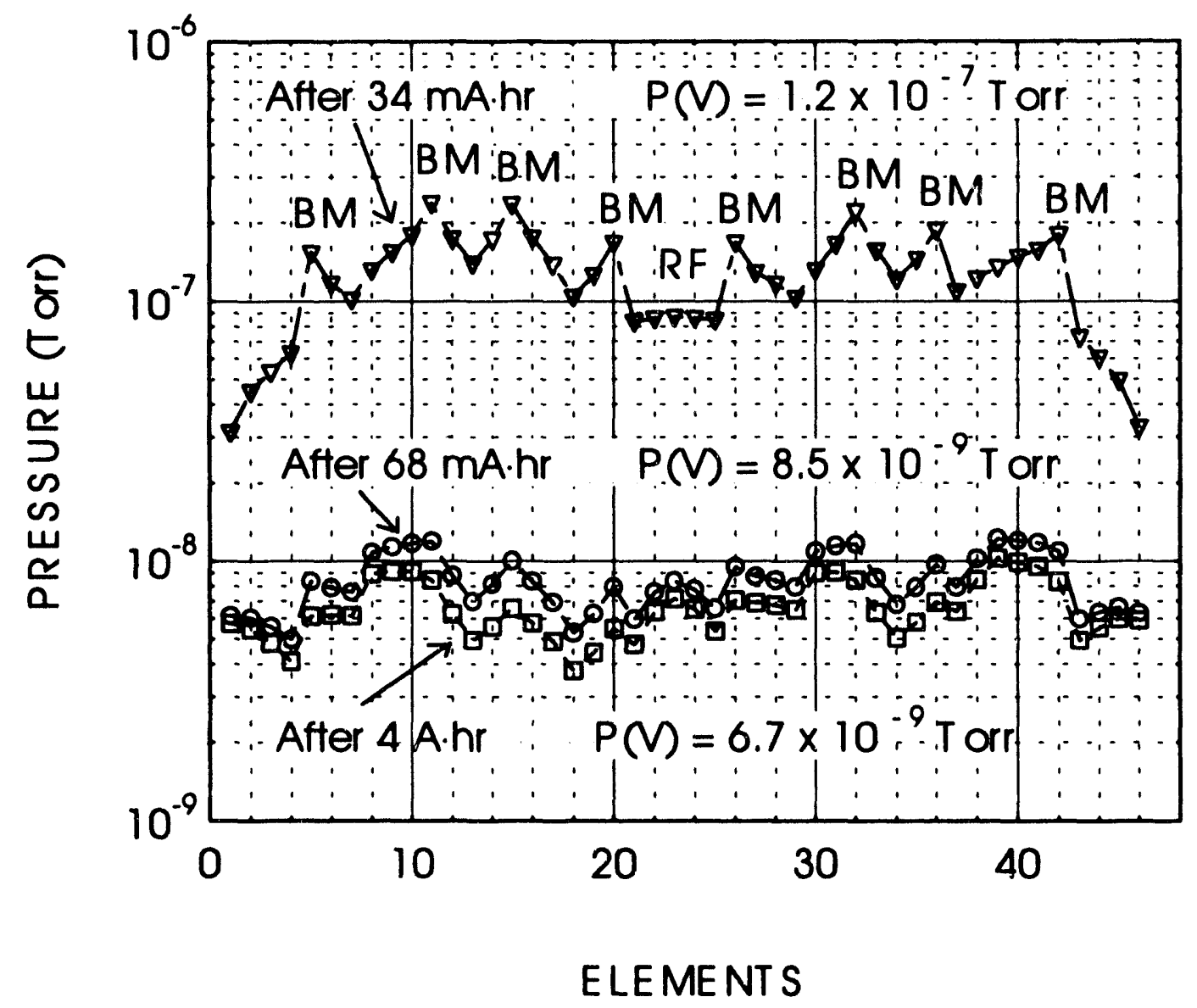

Figure 2.13 Pressure distribution along the positron accumulator ring. 


\subsection{Booster Synchrotron Ring}

The injector synchrotron system will boost the positron energy from $450 \mathrm{MeV}$ to $7 \mathrm{GeV}$. It is a $368-\mathrm{m}$ ring containing 40 vacuum chambers of various different types. ${ }^{13}$ Twenty-eight of the chambers are normal cells and ten other chambers are of varied sizes and shapes. They are enclosed within kicker magnets and septum magnets, in addition to dipoles. Two rf sectors contain rf cavities. Figure 2.14 shows the positions of these cells and the configuration of a normal cell.

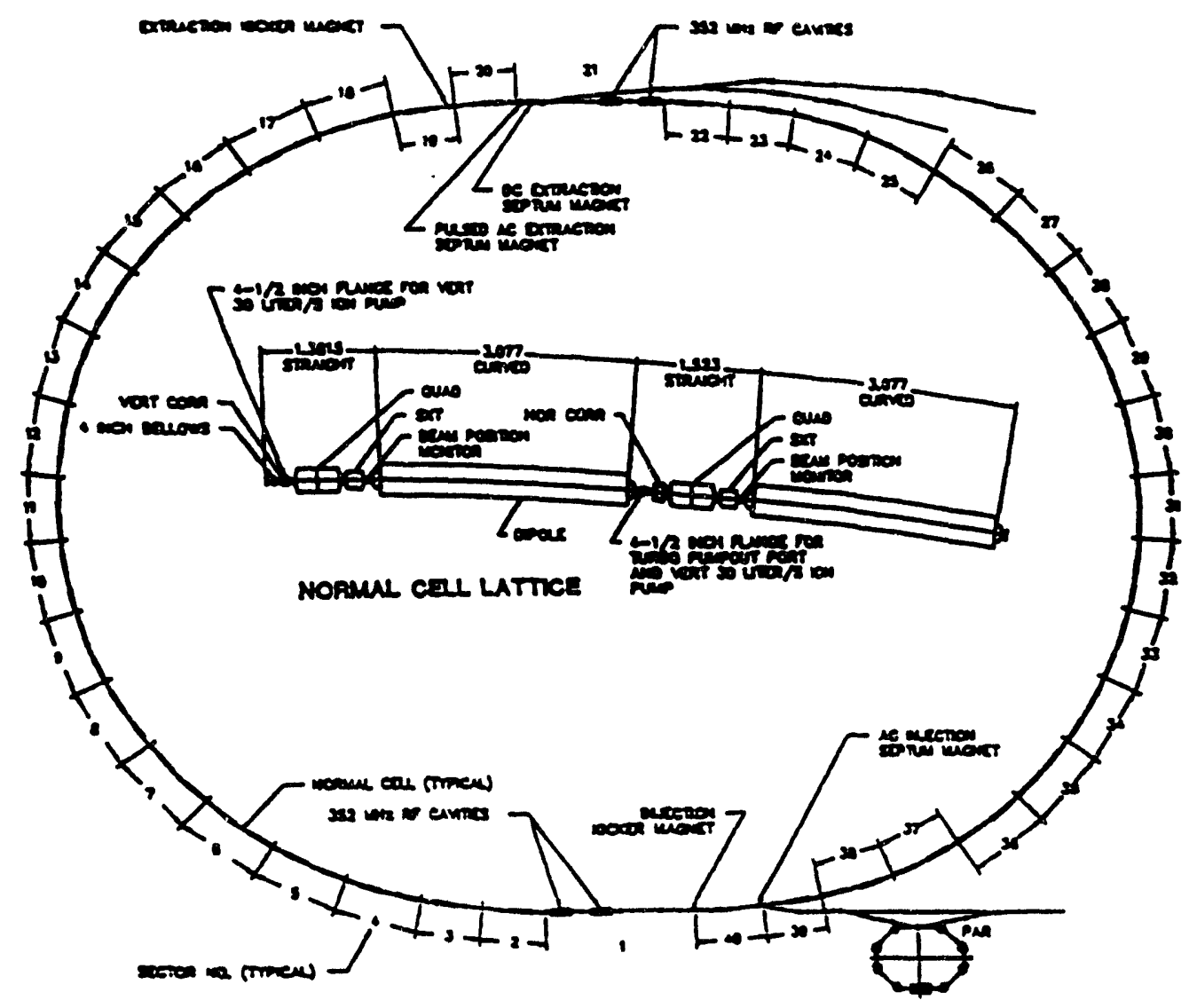

Figure 2.14 Layout of the booster synchrotron ring and the configuration of a normal cell (center).

The vacuum chambers are made from $316 \mathrm{~L}$ stainless steel tubing cold-drawn to an elliptical shape with inside major and minor axes of $6 \mathrm{~cm}$ and $3.7 \mathrm{~cm}$, respectively. The chambers are connected by bellows with Conflat-type flanges. In the sector of a normal 
cell there are dipole, quadrupole, sextupole, and correction magnets, beam monitors, bellows, and two ports for vacuum pumps (30-1/s ion pumps). The 30-1/s ion pumps are connected to the cells through a $21^{\prime \prime}$ pipe ( $\left.\phi 2.5^{\prime \prime}\right)$ and rf screen slots on the chamber opening, leading to an effective pumping speed of $18.5 \mathrm{Vs}$.

Sectors 1 and 21 contain a total of four 352-MHz rf cavities, which are powered by a single 1-MW klystron. ${ }^{11}$ A cylindrical ceramic window provides vacuum separation between the $\mathrm{rf}$ waveguide and the cavity. The cavity is a 5-cell cavity made of OFHC copper. Its $2.2-\mathrm{m}-\mathrm{long}, \phi 57.5-\mathrm{cm}$ cylinder contains a $\phi 10-\mathrm{cm}$ beam hole with a reentrant nose. The radius from the center line to the inside of the outer shell is $30.2 \mathrm{~cm}$. This structure has a large surface area. For the thermal outgassing rate of the cavity, we use a conservative value of $5 \times 10^{-11}$ Torr $\mathrm{V} / \mathrm{s}$ per $\mathrm{cm}^{2}$ because of the difficulty of an in situ bakeout of the cavity. In anticipation of a large gas load, two 400-1/s ion pumps are deployed in the rf sector. The effective pumping speed is $\sim 360 \mathrm{~V} / \mathrm{s}$ due to the conductance limitation of the 4 " orifice.

The $\sim 3.6 \times 10^{10}$ positrons dumped from PAR every half second are boosted from $450 \mathrm{MeV}$ to $7 \mathrm{GeV}$ in the injector synchrotron. One bunch of accelerated positrons are extracted per 0.5 -second cycle to the storage ring through the high energy transport line. The revolution time of positrons in the booster is $\sim 1.23 \mu \mathrm{s}$, leading to an average beam current of $4.8 \mathrm{~mA}$ with an average energy of $\sim 3.5 \mathrm{GeV}$. The high positron energy more than compensates the relatively small beam current to produce more photons than the PAR. The calculated photon yield is $\sim 1.67 \times 10^{19}$ photons per second, which is compared to $1.2 \times 10^{18}$ photons per second in the PAR. These photons are not uniformly distributed. We expect that the straight section in a normal cell will be subject to less radiation than the curved section (cf. Fig. 2.14). The if cavities and the portion immediately preceding them will have the least radiation, since they are longer straight sections. As a rough estimation, we assume a relative percentage of radiation per meter of $5 \%$ for the rf cavities, $10 \%$ for the portion immediately preceding the rf cavities in the if sector, and $50 \%$ for the straight sections in the other sectors.

The critical energy for the radiated photons varies according to the positron energy. At $3.5 \mathrm{GeV}$ and a $33.3-\mathrm{m}$ bend radius $\varepsilon_{\mathrm{c}} \cong 2.8 \mathrm{KeV}$. We still use Foerster et al.'s data 12 for stainless steel for our calculation as we did for PAR. That is, we use:

$$
\eta=\log ^{-1}(-2.7447-0.4184 \log x)
$$

and calculate $\mathbf{Q}_{\mathrm{iPD}}$ according to the photon distribution.

Because of the stronger photodesorption in the booster ring, we may assume the residual gas composition to be closer to that of the storage ring's (i.e., 75\% hydrogen and $25 \% \mathrm{CO}$ ). The calculated conductance is thus 3.4 times larger than that of air.

The booster ring has more or less a $180^{\circ}$ rotational symmetry, so that we need only calculate the pressure distribution of either the bottom half or the top half of the ring. We choose the bottom half, i.e., 20 sectors from Sector 31 to Sector 10 (cf. Fig. 2.14, clockwise). Sector 31 (the so-called equatorial section) has two curved parts and one straight part with one ion pump in the straight section. It is divided into three elements accordingly. Sectors 39,2 , and 3 are similar to Sector 31 and are thus divided into three elements each. Sector $\mathbf{4 0}$ has two ion pumps and is divided into four elements. Sector 1 
contains the rf cavities and other parts immediately preceding the cavities. The $\mathrm{rf}$ cavities are divided into five elements and the other portion into three. The remainder 14 sectors are normal cells. Each normal cell is divided into two straight elements plus two curved elements, with the ion pumps located in the straight elements. There are thus a total of 80 elements in our calculation.

Figure 2.15 shows the calculated pressure distribution along the bottom half of the booster ring after $240 \mathrm{~mA} \cdot \mathrm{hr}$ and $600 \mathrm{~mA} \cdot \mathrm{hr}$ of beam dose.

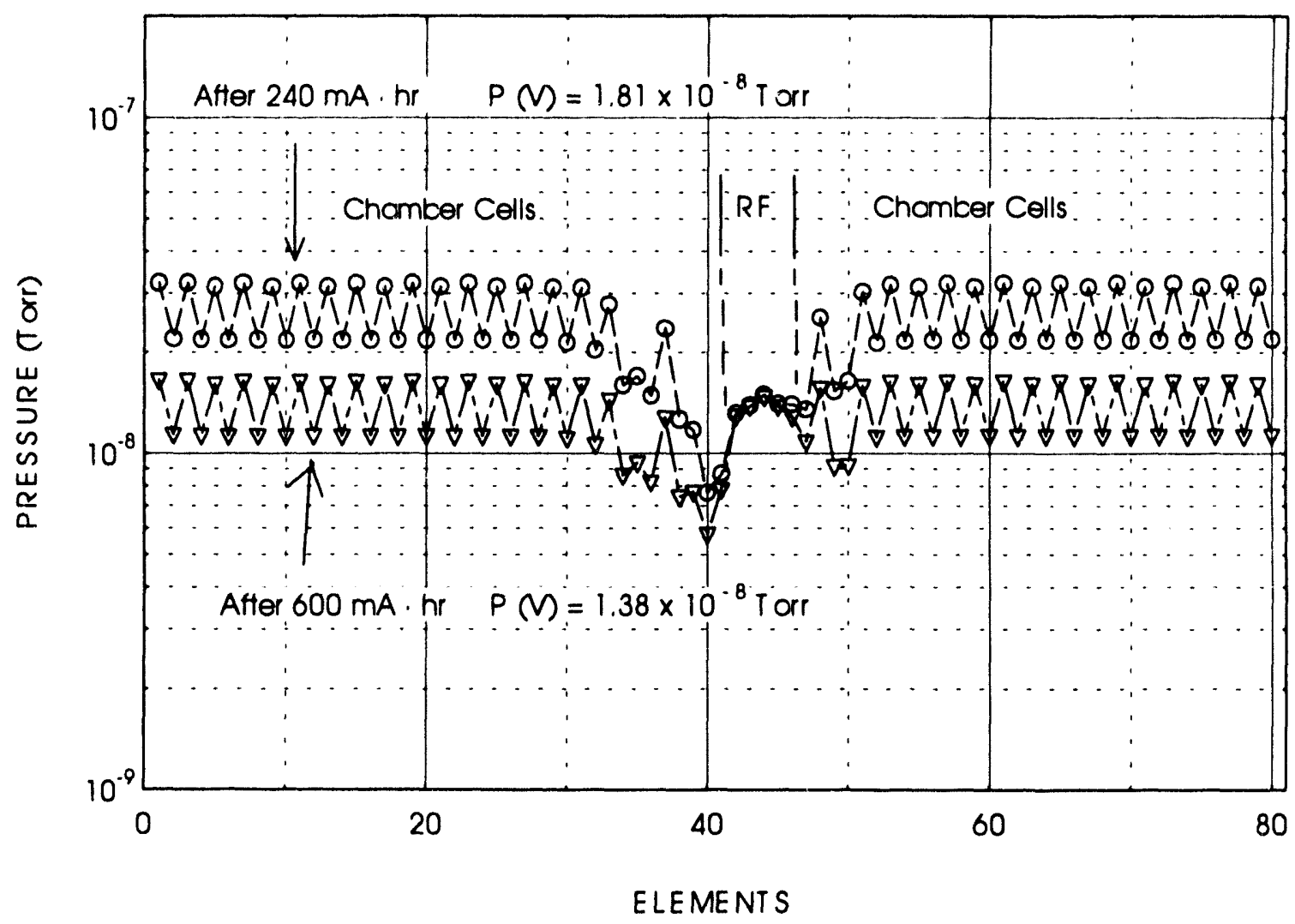

Figure 2.15 Pressure distribution along the bottom half of the booster ring after 240 $\mathrm{mA} \cdot \mathrm{hr}$ and $600 \mathrm{~mA} \cdot \mathrm{hr}$ of beam dose.

The vacuum requirement for the booster ring is better than $1 \times 10^{-7}$ Torr. Our calculation indicates that this requirement can be satisfied after four hours of commissioning at an average beam current of $4.8 \mathrm{~mA}$. After two days of operation the average pressure improves to $1.8 \times 10^{-8}$ Torr. From Fig. 2.15 one can see that those elements without ion pumps have a higher pressure due to the influence of limited conductance of the vacuum chamber. The vacuum at the rf cavities will remain at a low $10^{-8}$ Torr, while at other chamber cells the pressure will continue to decrease as the selfcleaning process proceeds. 
Since the positrons need to stay in the ring for only a very short time (half a second), the vacuum requirements for both PAR and the booster ring are very modest.

The situation in the storage ring is drastically different; this is discussed in the next section. 


\subsection{Storage Ring}

In contrast to PAR and the booster ring, the storage ring requires a much higher vacuum to fulfill the requirement of a good beam lifetime of at least 8 hours. The beam lifetime is defined as the time for the beam intensity to decay to the $e^{-1}$ of its initial value after injection. The better the vacuum, the longer the beam lifetime will be. The vacuum requirement for the storage ring is $1 \times 10^{-9}$ Torr with stored positron beam, and $1 \times 10^{-10}$ Torr without beam.

The vacuum calculations of the storage-ring have been performed at every major stage of the APS design. $1,11,14$ In the following we discuss only several issues which are not presented in previously referenced documents.

Because the photon dose in the storage ring is significantly larger than in PAR and the booster ring, the gas load due to photodesorption and its evolution with time are calculated differently in the storage ring. A fixed value of photodesorption yield $\eta$ is chosen for the chamber material after a substantial beam dose of $150 \mathrm{~A} \cdot \mathrm{hr}$. The initial photodesorption is determined backwards according to $Q_{0 P D}=Q_{P D} * t 0.63$. It is important to choose a reasonable value of $\eta$. The $\eta$ value of $2 \times 10^{-7}$ molecules per photon used in previous calculations is justified only for a well-prepared stainless steel chamber and may not be true for an aluminum one.

The storage ring vacuum chambers are made of 6063 aluminum. The noticeable difference between aluminum and stainless steel is that aluminum tends to develop oxide layers easily. The aluminum oxide may attract many layers of adsorbates which are released during photodesorption, leading to a higher photodesorption rate for aluminum than for stainless steel. Recently Mathewson et al. ${ }^{15}$ have carried out an experiment on an aluminum test chamber using photon doses ur, to $\sim 1.6 \mathrm{~A} \cdot \mathrm{hr}$. The measurements indicate a much higher $\eta$ value for aluminum than for stainless steel. A linear extrapolation (on the $\log$ scale) of their data to $150 \mathrm{~A} \cdot \mathrm{hr}$ leads to an $\eta$ value of $\sim 2 \times 10^{-6}$ molecules/photon, which is about 10 times higher than the $\eta$ value previously used in the vacuum calculation for the APS storage ring. However, a faster decrease of $\eta$ may occur at high photon doses, especially in cases where the initial photodesorption rate is unusually high. At present a value of $\eta=1 \times 10^{-6}$ molecule / photon at a beam dose of $150 \mathrm{~A} \cdot \mathrm{hr}$ is widely accepted.

To make sure that the present vacuum design of the APS storage ring is still satisfactory, we recalculated the pressure distribution along the storage ring. We have multiplied the $Q_{\text {iopD }}$ values used in the previous calculations by a factor of 6.5. i.e., we take $\eta=1.3 \times 10^{-6}$ molecules/photon at a beam dose of $150 \mathrm{~A} \cdot \mathrm{hr}$. The slightly larger $\eta$ value was used as an extra safety margin.

The crotch and distributed absorbers are introduced in the storage ring to prevent most of the unwanted photons from striking the vacuum chamber. We need to include them in our calculation.

The crotch absorber is newly designed. A water-cooled Glidcop plate with a special shape and geometry is designed to spread the intensity of the intercepted beam. The assembly of the crotch absorber is mounted on a 12" flange and its position is 
adjusted to meet the design requirement. Figure 2.16 is a drawing of the crotch-absorber chamber. 16

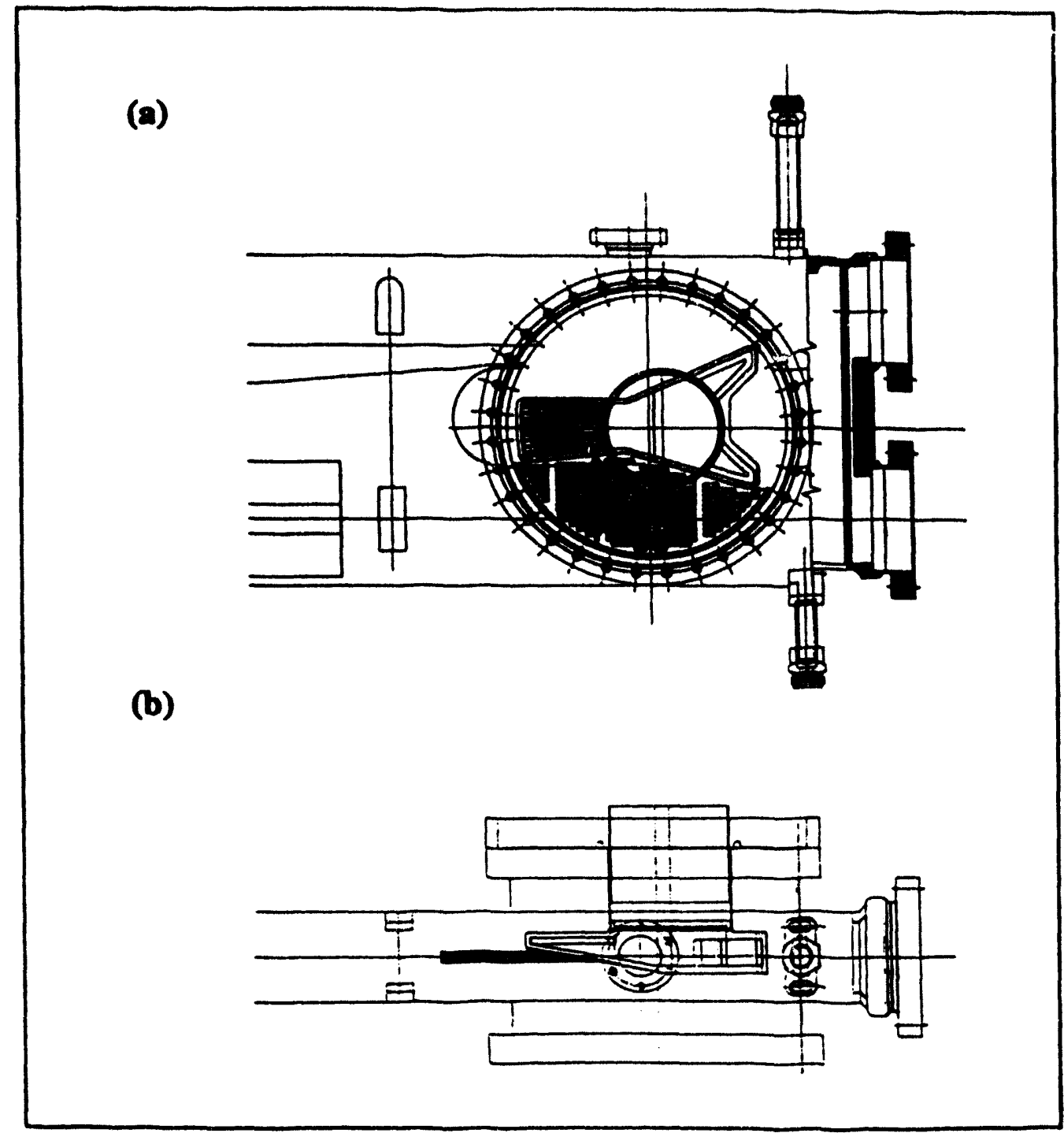

Figure 2.16 (a) A top view of the crotch-absorber chamber and (b) its side view showing the relative position of the crotch absorber with the photon beam.

Figure 2.16 (a) is a top view showing the 12" port opening, the rf screen with open slots, and the crotch absorber located in the center. Two rf screens (top and bottom ones) are needed to prevent the if power loss from the port opening. Fig. 2.16 (b) is a side view showing the relative position of the absorber to the photon beam.

The absorber is tilted vertically so that its bottom side is facing (at a grazing angle) the photon beam. Horizontally it is adjusted so that about $1 / 3$ of the intercepted power is absorbed by the side facing the positron-beam chamber. This position is an 
optimum choice for the power distribution on the absorber. It will also allow the majority of gases due to photodesorption to reach the open area in the absorber chamber, where the conductance is not limited by the slots on the rf screens.

The structure of the distributed absorbers (end absorbers) are similar to that of the crotch absorber except that their size is $\sim 40 \%$ smaller. They are mounted to an 8 " flange. The rf screen for the end absorber is also scaled down accordingly.

About $90 \%$ of the photodesorbed gas load will come from the crotch and distributed absorbers. The photodesorption rate for Glidcop is unknown but it can be safely assumed to be no larger than that of aluminum.

A lumped NEG pump with a nominal pumping speed of $1000 \mathrm{l} / \mathrm{s}$ is added on the top of a 220-1/s ion pump to pump the crotch-absorber chamber. The conductance of the lumped NEG pump is calculated to be $\sim 480 \mathrm{l} / \mathrm{s}$, which limits the effective pumping speed for the ion pump to $150 \mathrm{l} / \mathrm{s}$. For the end absorber, a 250-1/s NEG pump is used with a $220-1 / \mathrm{s}$ ion pump.

The rf screens in the absorber chamber reduce the effective pumping speed drastically. The effective pumping speed used in the present calculation is less than $1 / 3$ of that previously used. Fortunately the rf screens affect only the positron chamber. Furthermore, only about $1 / 3$ of the photodesorbed gases will be trapped inside the positron-beam chamber; the other $2 / 3$ will be pumped directly by the lumped pumps.

In the previous calculation of the conductance, the residual gas in the storage ring is assumed to be $100 \%$ hydrogen. A composition of $75 \%$ hydrogen and $25 \% \mathrm{CO}$ is more likely. We have decreased the value of conductance for each element accordingly.

The results of vacuum calculations are shown in the next section, together with the results for the insertion devices. 


\subsection{Insertion devices}

The primary purpose of APS is to provide radiation from the insertion devices. Insertion devices, mainly undulators and wigglers, were incorporated into the storage ring from the very beginning of the APS design. These devices provide many advanced features for APS, such as tunability, ultrahigh spectral brilliance or brightness, specific polarization characteristics, extremely low divergence, and very small beam size (a few microns), etc. These advanced features are crucial for the success of APS.

Undulators and wigglers are basically systems consisting of periodic magnets. The vacuum chambers within the insertion magnets have a small vertical size in order to economically obtain the high magretic field required. Distributed NEG pumps are needed.

Figure 2.17 shows the cross section of an undulator vacuum chamber. Two NEG strips will be installed in the antect.amber to provide a nominal pumping speed of $\sim 400$ $1 / s$ per meter. The vertical height of the beam chamber is $12 \mathrm{~mm}$ and the gap in the channel connecting the beam chamber and the antechamber is $8 \mathrm{~mm}$. In a later stage the gap is going to be reduced to only $6.35 \mathrm{~mm}$ and the vertical height to $8 \mathrm{~mm}$.

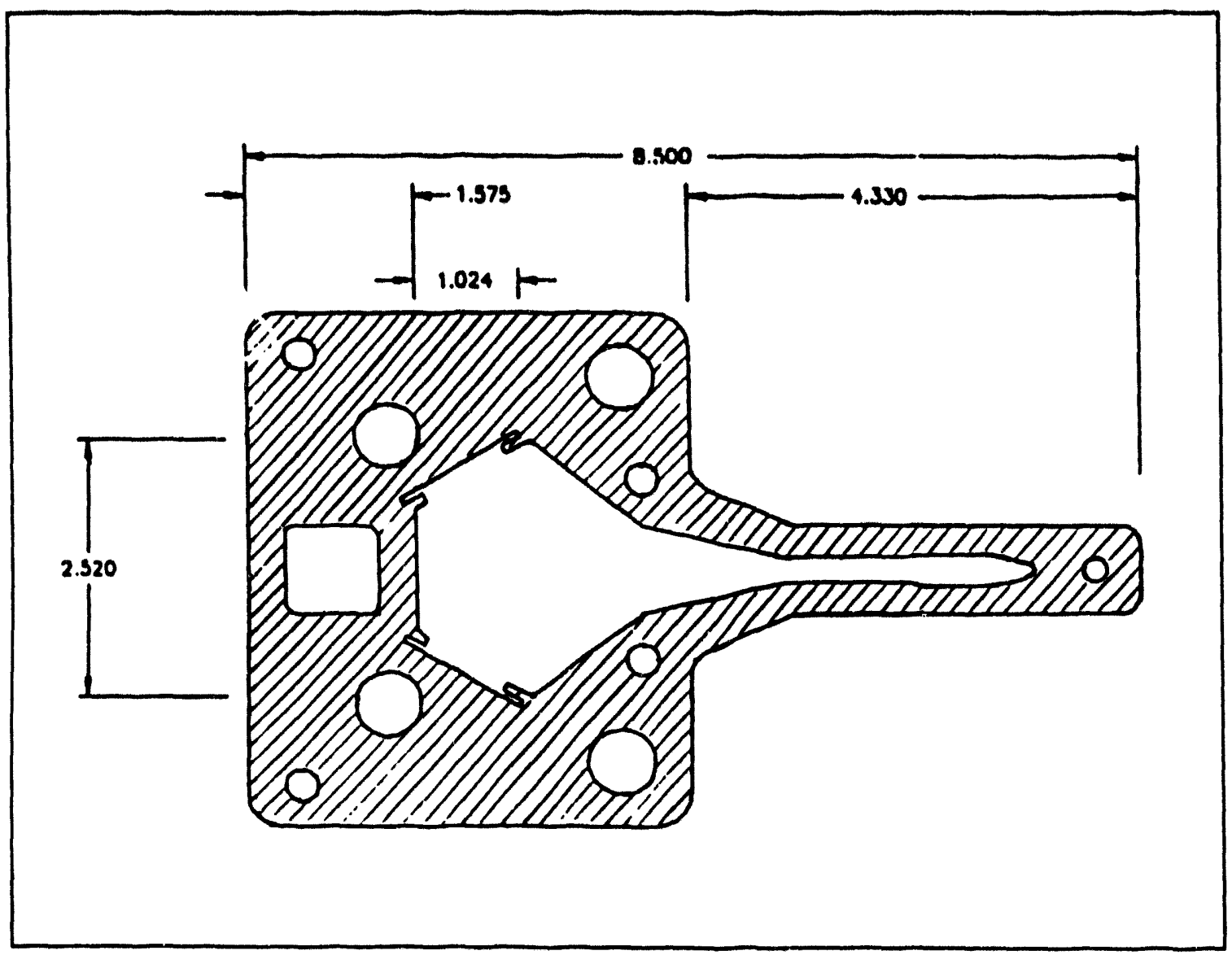

Figure 2.17 The insertion device vacuum chamber cross section. Numbers indicate dimensions in inches. 
The storage ring is divided into 40 sectors. Each sector is again divided into six sections. Section 6 is a 5.2-m straight section, which can accommodate two insertion devices, each $2.4 \mathrm{~m}$ long. Three bellows and transition parts connect them with other sections. In the case where only one insertion device is needed, an ordinary storage ring vacuum chamber is used in place of the first insertion device.

In this straight section there are two absorbers, one in the center and another at the end. The distribution absorber in Section 5 will block most of the radiation and let only $7.2 \mathrm{mrad}$ (out of $2 \pi$ ) of the radiation pass through to the insertion device. The center absorber in Section 6 will let $4 \mathrm{mrad}$ of radiation pass. The end absorber will block all the remaining radiation when needed. The absorter is contained in a box-shaped vacuum chamber which is pumped in parallel by a 30-1/s ion pump and a 220-1/s NEG pump. The ion pump is needed to pump those gases which cannot be efficiently pumped by NEG pumps, such as methane $\left(\mathrm{CH}_{4}\right)$ and argon.

The photon-stimulated desorption in the insertion device section occurs mostly in the absorber chambers. The rest of the insertion device vacuum chamber is assumed to have only $5 \%$ of the $7.2 \mathrm{mrad}$ radiation distributed uniformly along the $5.2 \mathrm{-m}$ section. Again, we assume a $\eta$ value of $1.3 \times 10^{-6}$ molecules/photon at a beam dose of $150 \mathrm{~A} \cdot \mathrm{hr}$. The amount of photodesorption for each part is calculated according to the intercepted radiation power.

Section 6 is divided into eight elements. Together with the 27 elements in Sections 1 through 5, there are 35 elements used in our vacuum calculation for one sector of the storage ring. Figure 2.18 shows the new result of the calculated pressure distribution for the case where the insertion device section contains one ordinary storage ring vacuum chamber and one insertion device chamber. The insertion device chamber has a channel gap of $8 \mathrm{~mm}$ and a vertical height in the beam chamber of $12 \mathrm{~mm}$.

We summarize the changes we made from the previous calculations as follows. In our calculation, we have increased the $\eta$ value (and thus the photodesorption) by a factor of 6.5 , decreased the conductance for each element by a factor of 3.4/3.8, included the influence of the rf screen on the effective pumping speed, and added the insertion device section.

From Figure 2.18 we conclude that the required vacuum can be achieved after a beam dose of $\sim 10 \mathrm{~A} \cdot \mathrm{hr}$. This translates to a conditioning period of about five days with an average beam current of $100 \mathrm{~mA}$. The thermal desorption rate of aluminum is assumed to be $3 \times 10^{-12}$ Torr $1 / \mathrm{s}$ per $\mathrm{cm}^{2}$. This low desorption rate requires very careful cleaning and handling of the vacuum chamber. An in situ bakeout is also required to decrease the water content in the system, since the synchrotron radiation is not efficient in removing water molecules. No detectable vacuum leak is allowed in the system.

In a later stage it will become necessary to design an insertion device with a beam chamber vertical height of $8 \mathrm{~mm}$ and a channel gap of $6.35 \mathrm{~mm}$. A major concern is whether the achievable vacuum is still adequate. The main limitation is the smaller conductance in both the beam direction and the transverse direction to the NEG distributed pump. The worst condition will occur when two insertion devices with narrow gaps are installed in the insertion device section. 


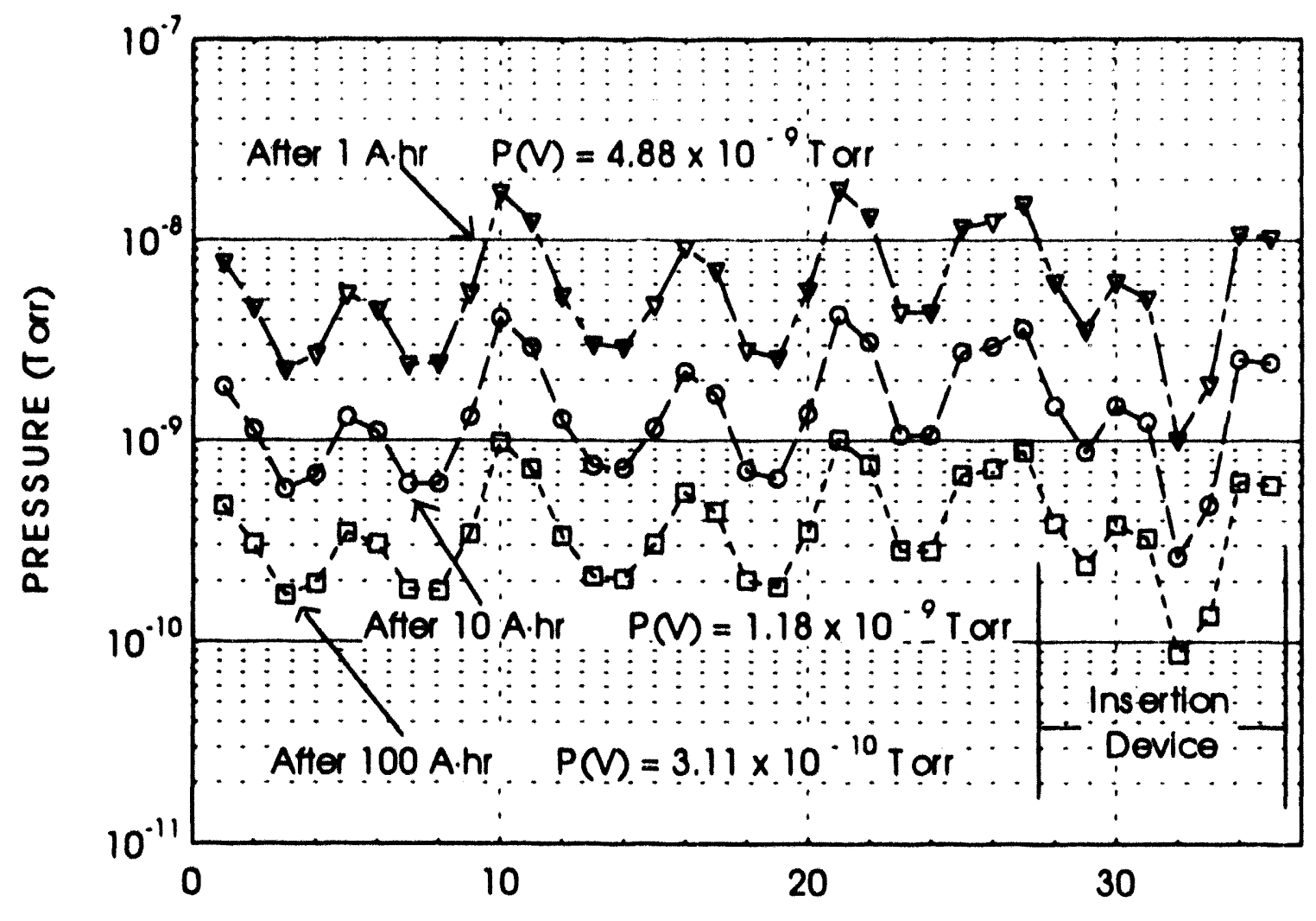

\section{ELEMENTS}

Figure 2.18 Pressure distribution along one sector of the storage ring. The insertion device section contains one insertion device chamber and one ordinary storage ring chamber.

The transverse pressure distribution is calculated by dividing the cross section of the insertion chamber (cf. Fig. 2.17) into four elements, namely, the antechamber, the section connecting the antechamber and the channel, the channel gap, and the beam chamber. We assume a segment of $10 \mathrm{~cm}$ of the chamber for our calculation. Figure 2.19 shows the transverse pressure profiles for the two designs of the insertion chamber, i.e., (a) a channel gap of $6.35 \mathrm{~mm}$ and vertical height of $8 \mathrm{~mm}$ and (b) a gap of $8 \mathrm{~mm}$ and height of $12 \mathrm{~mm}$.

Figure 2.19 demonstrates that the difference in pressure for these two cases is not substantial. The calculation included only $5 \%$ of the $7.2-\mathrm{mrad}$ radiation. The photodesorption mainly occurs in the absorber chambers, where the conductance is not limited by the channel gap. The limited conductance in the channel gap is helped by the gas content, which is mainly hydrogen. The initial pump down of the insertion chamber will take longer when the channel gap is small. 


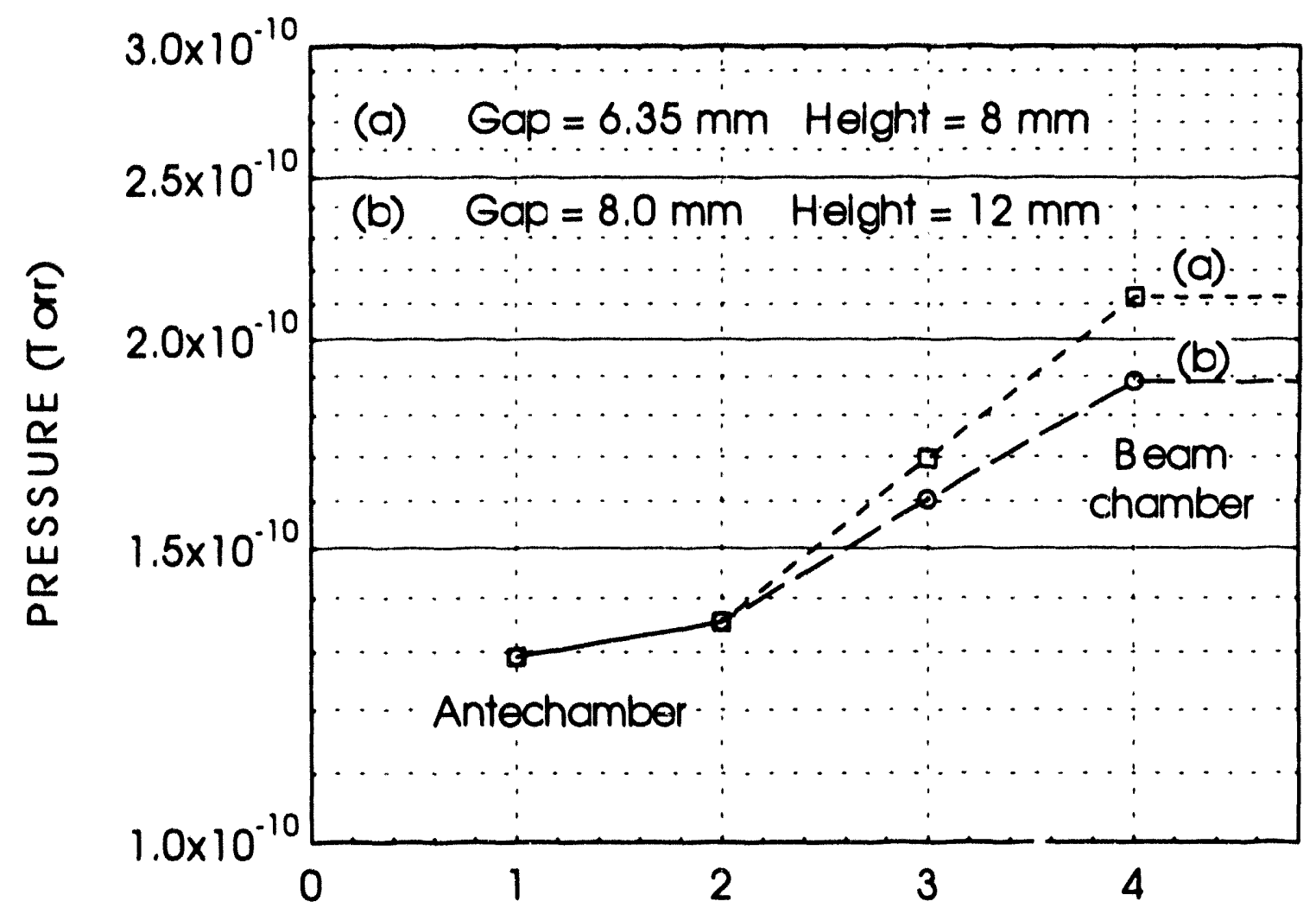

ELEMENT S

Figure 2.19 Pressure profile across the insertion chamber for two designs with different dimensions.

To summarize, the present design of the APS vacuum system is appropriate for satisfactory operation. To achieve the designed ultrahigh vacuum, we now need to make sure that every step of manufacturing and assembling is carried out properly. One of the most important steps is cleaning. In the next chapter we discuss cleaning procedures. 


\section{Cleaning Procedures for Ultrahigh Vacuum Components}

Every component placed in ultrahigh vacuum must be thoroughly cleaned before assembling and installation. The result of component cleanliness in APS will be reflected in the beam stability and ultimate vacuum achieved. For example, diminutive amounts of contamination, such as oils, greases, flux, marking inks and paint, and fingerprints, can keep the designed ultrahigh vacuum from being reached in a reasonable time. Loose foreign particles, even a tiny piece of lint, when hit by a high energy particle, will cause a burst in pressure and result in beam instability.

We have two major concerns regarding APS cleaning procedures: the general cleaning methods to remove traces of molecular and particulate contaminants and the special concern related to the photon-stimulated desorption. Both issues are still research projects under investigation. In the following we discuss them separately.

Cleaning processes have been studied for many years as an integral part of vacuum technology. Satisfactory cleaning recipes for almost every kind of material have been devised over the years (many of them can be found in Rosebury's book ${ }^{17}$ ). However, the majority of these recipes involve the use of organic solvents which may impose environmental and safety hazards. For example, methyl chloroform $(1,1,1$ trichloroethane) and chlorofluorocarbons (e.g. CFC-113) are ozone-depleting materials which should be gradually phased out according to the Montreal Protocol. These and other organic solvents such as trichloroethylene and perchloroethylene are all volatile organic compounds (VOCs). VOCs are flammable materials and may have other safety concerns. Finding replacement cleaning recipes is thus an important research project and has received considerable attention.

Although the cleaning recipes need to he revised, the general purpose of cleaning remains the same, i.e., to remove molecular and particulate contaminations. The materials used for APS vacuum chambers are mainly 6063 aluminum alloy, stainless steel, and copper. Some flanges are made of 2219 aluminum alloy. Experiments conducted by the ASD Vacuum Group using $x$-ray photoelectron spectroscopy (XPS) indicate that simple alkaline or acidic detergents in an ultrasonic bath followed by deionized (DI) water rinse can effectively clean these materials. ${ }^{18}$

In addition to the general concerns of cleanliness, special attention should be paid to oxide layers and carbon content on the APS storage ring chamber due to the photonstimulated desorption. As we discussed in Chapter 1, loosely adherent layers of porous oxides can be a nightmare for the APS vacuum. An ideal cleaning process should produce a very thin, tightly bonded, non-porous aluminum oxide layer. Any carbon present on the surface will react with oxygen and hydrogen. The resulting $\mathrm{CO}, \mathrm{CO}_{2}$, and $\mathrm{CH}_{4}$ are main residual-gas components in a typical UHV system, in addition to hydrogen and water. A good cleaning process should reduce the carbon content. The 6063 aluminum alloy consists of $\mathrm{Al}, 0.4 \% \mathrm{Si}$, and $0.7 \% \mathrm{Mg}$. The 2219 aluminum has $6.3 \% \mathrm{Cu}$ and $0.3 \% \mathrm{Mn}$. ${ }^{19}$ Care should be taken in the cleaning process to avoid possible chemical reactions between the alloy components and the cleaning agent. 
The following are recipes developed through XPS studies for 6063 and 2219 aluminum alloys and copper, 18 and general cleaning and assembling procedures presently used at APS.

\section{Aluminum}

1. Preclean with a high pressure spray of $2 \%$ Almeco 18.

2. Ultrasonically clean with a $2 \%$ solution of Almeco 18 at $65^{\circ} \mathrm{C}$ for 10 minutes.

3. Rinse with room temperature flowing deionized (DI) water for 10 minutes.

4. Blow dry with hot, dry nitrogen.

\section{Aluminum}

1. Ultrasonically clean with a $2 \%$ solution of Almeco 18 at $50^{\circ} \mathrm{C}$ for 10 minutes.

2. Rinse with room temperature flowing DI water for 10 minutes.

3. Blow dry with hot, dry nitrogen.

\section{Copper}

1. Ultrasonically clean with a $2 \%$ solution of Citranox at $65^{\circ} \mathrm{C}$ for 10 minutes.

2. Rinse with room temperature flowing DI water for $\mathbf{1 0}$ minutes.

3. Blow dry with hot, dry nitrogen.

\section{Cleaning Procedure General Guidelines:}

1. Removal of large contaminants with one or the combination of the following methods:

- Mechanical removal through scraping, brushing, and wiping with stainless steel tools only.

- Swabbing with a cloth soaked in alcohol, freon, or trichloroethane.

- Glass bead blasting, using fresh beads.

2. Vapor degreasing in trichloroethane followed by draining and drying.

3. Soak in hot, non-etch alkaline detergent with ultrasonic treatment.

4. Rinsing in deionized (or demineralized) water.

5. Drying in a hot air oven. 
Alcohol soak instead of detergent soak should be used to clean welded bellows, ferrites, feed-throughs, and ceramics with brazed metal parts.

Acid or alkaline etch/pickling to remove the scale or the oxide layer is allowed only when they are specified or approved.

No etching is allowed after welding or brazing.

\section{Clean Assembly Procedures:}

1. Assembly, welding, inspection and packing of the components should take place in a dedicated clean area. This area should be maintained in a smokeand dust-free environment. It is very important that the areas should be isolated from machine tools, compressors, mechanic pumps, oily or dirty parts and tools, etc., which can contaminate the work area with hydrocarbons and dirt. Eating, drinking, and smoking are prohibited in the clean area.

2. Clean components shall be handled only with clean white nylon or cotton gloves. Clean lab coats are needed in the clean area to reduce the lint and dust density in air. Clean gloves should only contact clean parts and tools in the clean work area. If the gloves touch unclean surfaces, e.g., hair, face, chair, floor, door knobs, and uncleaned tools, they should be replaced.

3. All tools and inspection gauges that contact clean components shall be precleaned and wiped with alcohol using lint-free cloth or paper to ensure no contamination (oil or dirt) will be transferred to the parts or the inspector's gloves. When the tools are not in use, they should be covered and stored to prevent their use on dirty parts. Tools may not be made of material which can contaminate the clean parts, etc.

4. Work bench surfaces must be either thoroughly precleaned with detergent, rinsed with water, and then wiped with alcohol using lint-free, low sulfur paper, or protected with clean coverings such as cleanroom grade TYVEK or oil-free Al foil. The surface must be hard and non-absorbent (stainless steel, formica, etc.). Wood bench tops or similar materials are not acceptable.

5. The welded, or assembled, components shall be kept covered and ports wrapped in clean, oil-free $\mathrm{Al}$ foil and protected when the components are not undergoing work or inspection. 
6. Blowing the components with compressed gas should be avoided. If compressed gas is used, it must be dry, oil-free, and filtered.

The above procedures serve as general guidelines. Precise procedures depend on the material and the adopted fabrication and assembly techniques. Detailed instructions should be followed. 


\section{Summary}

In this document we have presented a brief discussion of basic ultrahigh vacuum technology as it applies to the APS.

This document contains up-to-date information about the vacuum design of most major parts of the APS, including the linac, the undulator test line, PAR, the booster ring, insertion devices, and the storage ring. Pressure profiles have been calculated using finite element analyses and Monte Carlo simulations. These results can be used to analyze potential vacuum problems during the construction stage and to monitor the system performance during the operation of the APS. One can find the expected pressure readings from the calculated pressure distributions.

Cleaning procedures of ultrahigh vacuum components are included as general guidelines.

We hope that this document will be a useful vacuum guide for both APS personnel and general users.

\section{Acknowledgments}

We wish to thank members from the APS Linac, RF, Mechanical Engineering, Vacuum, Design and Drafting, and Insertion Devices Groups for information needed in writing this document. Special thanks are due to Ralph Benaroya, Richard Rosenberg, and Bob Nielsen for critical reading of the manuscript. We appreciate the editing effort by Cathy Eyberger. 


\section{Bibliography}

The following books on vacuum (in chronological order) have been consulted in preparing the present document:

Handbook of Electron Tube and Vacuum Techniques (Addison-Wesley, Reading, 1965), by F. Rosebury.

Vacuum Manual (Spon, London, 1974), by L. Holland, W. Steckelmacher and J. Yarwood.

Vacuum Physics and Technology (Methods of Experimental Physics; v. 14), (Academic Press, London, 1979), edited by G. L. Weissler $e t$ al.

Ultrahigh Vacuum Practice (Butterworths, London, 1985), by G. F. Weston.

A User's Guide to Vacuum Technology, 2nd edition (John Wiley \& Sons, New York, 1989), by J. F. O'Hanlon.

Vacuum Technology, 3rd edition (North-Holland, Amsterdam, 1990), by A. Roth.

\section{References}

1. G. K. Shenoy, P. J. Viccaro, and D. M. Mills, "Characteristics of the 7-GeV Advanced Photon Source: A Guide for Users," Argonne National Laboratory Report ANL-88-9 (Feb. 1988);

"7-GeV Advanced Photon Source: Conceptual Design Report", ANL-87-15 (April 1987).

2. C. Liu and S. D. Bader, Phys. Rev. B44, 2205 (1991).

3. H. F. Dylla et al., J. Vac. Sci. Technol. 17, 286 (1980); A. G. Mathewson et al., Proc. VIIth Int. Vac. Congress, Vienna, 1027 (1977).

4. D. Rydin, private communication.

5. C. A. Melendres, private communication.

6. J. F. J. van der Brand and A. P. Kaan, "Design Study of the Vacuum System for the E. S. R. F.," European Synchrotron Radiation Project Report ESRP-IRM61/84 (1984).

7. J. Kneuer, unpublished information (1985).

8. R. Kersevan, "MOLFLOW User's Guide," Sincrotrone Trieste Technical Report ST/M-91/17 (1991).

9. R. Haefer, Vacuum, 30, 217 (1980).

10. Z. D. Farkas et al., "SLED: A Method of Doubling SLAC's Energy," SLAC-PUB 1453, June 1974; IEEE Trans. Nucl. Sci., NS-24, 1827 (1977). 
11. "Annex to $7 \mathrm{GeV}$ Advanced Photon Source Conceptual Design Report," ANL-8715 Annex, May 1988.

12. C. L. Foerster et al., J. Vac. Sci. Technol., A10, 2077 (1992).

13. R. Benaroya and R. Dortwegt, Proc. Particle Accelerator Conf., V4, 2275 (1991).

14. APS Design Handbook, Dec. 1989.

15 A. G. Mathewson, Synchrotron Radiation News Compendium, Y1-3, 333 (1990).

16. J. W. Howell, private communication.

17. F. Rosebury, Handbook of Electron Tube and Vacuum Techniques (AddisonWesley, Reading, 1965).

18. R. A. Rosenberg et al., preprint, the American Vacuum Sociaty 40th Symposium, 1993.

19. "Aluminum Standards and Data", The Aluminum Association, p. 15, 1990. 
Appendix: Computer Program for Calculating Pressure Profiles along PAR

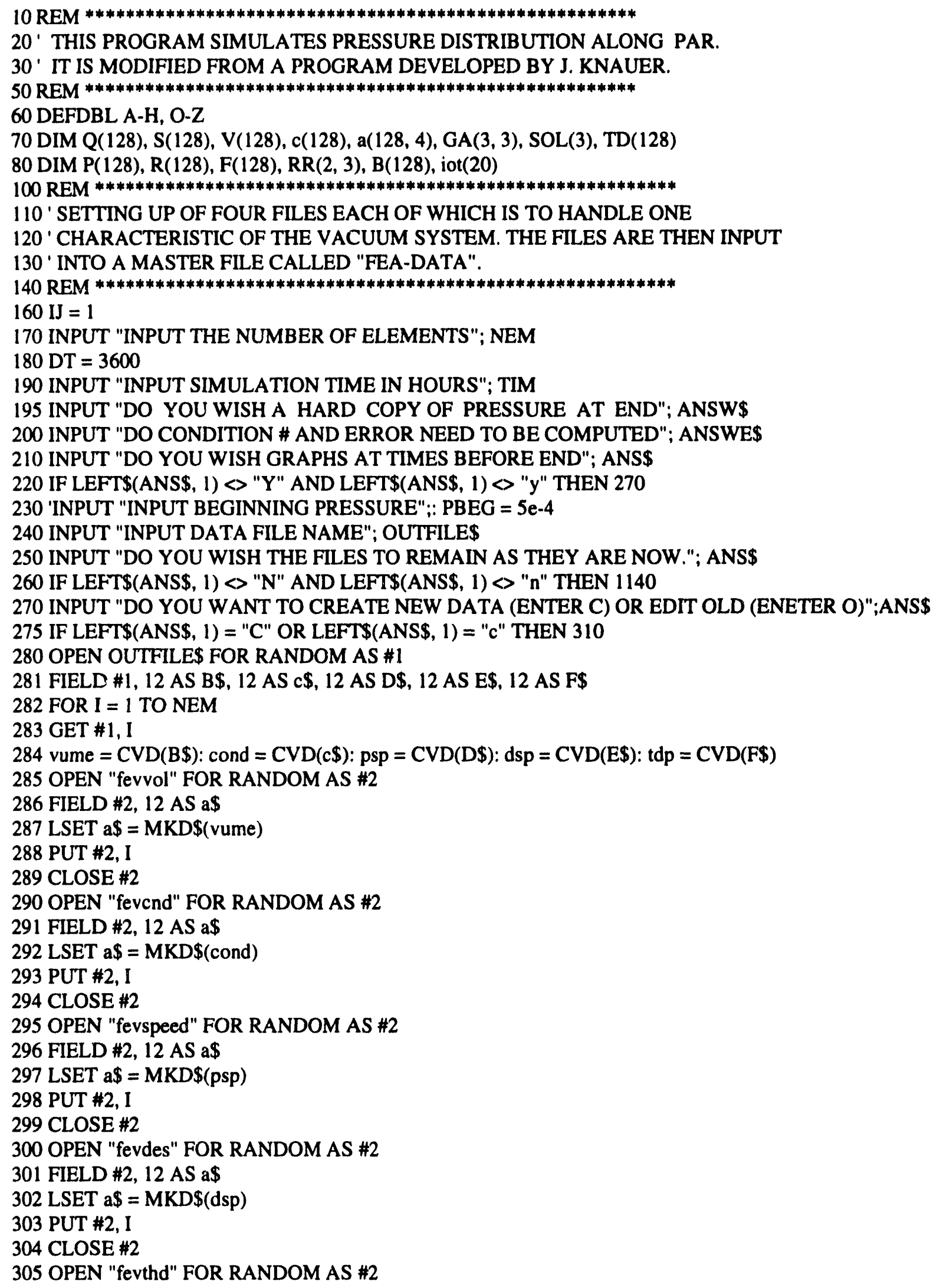


306 FIELD \#2, 12 AS a\$

307 LSET a $\$=$ MKD $\$(t d p)$

308 PUT \#2, I

309 CL.OSE \#2: NEXT I: CLOSE \#1

310 ILS : PRINT "ENTER \# OF FILE YOU WISH TO EDIT"

320 PRINT "1 VOLUME"

330 PRINT "2 CONDUCTANCE"

340 PRINT " 3 PUMPING SPEED"

350 PRINT "4 DESORPTION"

355 PRINT "5 THERMAL DESORPTION"

360 PRINT "6 EXIT FROM MENU"

370 INPUT ICHOICE

380 IF ICHOICE = 1 THEN B $\$=$ "FEVVOL": BB $\$=$ "VOLUME": GOTO 430

390 IF ICHOICE $=2$ THEN B $\$=$ "FEVCND": BB $\$=$ "CONDUCTANCE": GOTO 430

400 IF ICHOICE = 3 THEN B $\$=$ "FEVPSPEED": BB $\$=$ "PUMPING SPEED": GOTO 430

410 IF ICHOICE $=4$ THEN B $\$=$ "FEVDES": BB $\$=$ "DESORPTION": GOTO 430

415 IF ICHOICE = 5 THEN B $\$=$ "FEVTHD": BB $\$=$ "THERMAL DES.": GOTO 430

420 GOTO 801

430 OPEN B\$ FOR RANDOM AS \#2: FIELD \#2, 12 AS a\$

440 PRINT "DO YOU WISH TO ENTER "; BB\$; " SEQUENTIALLY"; : INPUT ANS\$

450 IF LEFT\$(ANS\$, 1) = "N" OR LEFT\$(ANS\$, 1) = " $n$ " THEN 570

460 PRINT "ENTER RANGE TO BE ENTERED SEQUENTIALLY WITH THE TWO NUMBERS"

470 INPUT "SEPERATED BY A COMMA"; IBEG, IEND

480 IF IEND > NEM THEN PRINT "RANGE TO BIG:GO TO 380"

490 FOR I = IBEG TO IEND

500 PRINT BB\$; " OF ELEMENT"; I: INPUT HOLD

$510 \quad$ LSET a $\$=$ MKD $\$($ HOLD)

520 PUT \#2, I

$530 \quad$ NEXT 1

540 INPUT "ANY MORE DATA TO BE ENTERED SEQUENTIALLY"; ans\$

550 IF LEFT\$(ANS\$, 1) = "Y" OR LEFT\$(ANS\$, 1) = " $y$ " THEN 460

560 GOTO 650

570 PRINT "ENTER 0 FOR ELEMENT \# WHEN THROUGH"

$580 \mathrm{M} \$=$ "INPUT " + BB \$

590 INPUT "INPUT ELEMENT \#"; ELEMNO

600 IF ELEMNO $=0$ THEN 650

610 PRINT M\$: INPUT HOLD

620 LSET a $\$=$ MKD $\$($ HOLD)

630 PUT \#2, ELEMNO

640 GOTO 590

650 INPUT "DO YOU WISH PRINTOUT"; ANS\$

660 IF LEFT\$(ANS\$, 1) = "N" OR LEFT\$(ANS\$, 1) = "n" THEN 750

661 PRINT "INPUT RANGE TO BE PRINTED OUT. PROGRAM WILL LOOP"

662 PRINT "BACK TO PRINT OTHER RANGES IF YOU SO DESIRE. IF ONLY A "

663 PRINT "SINGLE NUMBER IS TO BE PRINTED ENTER IT TWICE.";

664 INPUT IBEG, IEND

690 FOR I = !BEG TO IEND

$700 \quad$ GET \#2, I

710

720

730

740

750

760

770

PRINT I; : PRINT USING "\#.\#\#^^^^"; CVD(a\$) NEXT I

INPUT "IS THERE ANOTHER RANGE TO BE PRINTED"; ANS\$

IF LEFT\$(ANS\$, 1) = "Y" OR LEFT\$(ANS\$, 1) = "y" THEN 661

INPUT "ANY ALTERATIONS STILL NEEDED ON THIS FILE"; ANS\$

IF LEFT\$(ANS\$, 1) = "Y" OR LEFT\$(ANS\$, 1) = "y" THEN 440 ELSE CLOSE \#2

GOTO 310 
801 INPUT "DID YOU ALTER ANY FILES"; ANS\$

IF LEFT\$(ANS\$, 1) <> "Y" AND LEFT\$(ANS\$, 1) <> "y" THEN 1140

PRINT "PROGRAM NOW CREATES MASTER FILE FEA-DATA"

790 OPEN OUTFILES FOR RANDOM AS \#1

800

FIELD \#1, 12 AS B\$, 12 AS c\$, 12 AS D\$, 12 AS E\$, 12 AS F\$

810

820

830

840

850

860

870

880

890

900

910

920

930

940

950

960

970

980

990

1000

1010

1020

1030

1040

1050

1060

1070

1080

1081

1082

1083

1084

1085

1090

1100

1110

1120

1121

1122

1130 CLOSE \#1

\section{PRINT "INPUT}

PRINT "THIS WILL CORRESPOND TO THE NUMBERS OF THE ELEMENTS WHICH WERE" PRINT "EDITED BEFORE EXITING THE MENU. IF THE NUMBERS OF THE ELEMENTS"

PRINT "WHICH WERE EDITED WERE NOT IN SEQUENCE THEN ENTER THE ELEMENT \#"

PRINT "AS BOTH THE BEGINNING AND ENDING. THE PROGRAM LOOPS BACK TO GET"

INPUT "THE OTHER CORRECTIONS."; IBEG, IEND

IF IEND > NEM THEN PRINT "RANGE TOO BIG": GOTO 810

FOR I = IBEG TO IEND

OPEN "FEVVOL" FOR RANDOM AS \#2

FIELD \#2, 12 AS a\$

GET \#2, I

vume $=\mathrm{CVD}(\mathrm{a} \$)$

CLOSE \#2

OPEN "FEVCND" FOR RANDOM AS \#2

FIELD \#2, 12 AS a\$

GET \#2, I

cond $=\mathrm{CVD}(\mathrm{a} \$)$

CLOSE \#2

OPEN "FEVPSPEED" FOR RANDOM AS \#2

FIELD \#2, 12 AS a\$

GET \#2, I

$\mathrm{psp}=\mathrm{CVD}(\mathrm{a} \$)$

CLOSE \#2

OPEN "FEVDES" FOR RANDOM AS \#2

FIELD \#2, 12 AS a\$

GET \#2, I

$\mathrm{dsp}=\mathrm{CVD}(\mathrm{a} \$)$

CLOSE \#2

OPEN "FEVTHD" FOR RANDOM AS \#2

FIELD \#2, 12 AS a $\$$

GET \#2, I

$\mathrm{tdp}=\mathrm{CVD}(\mathrm{a} \$)$

CLOSE \#2

LSET B $\$=$ MKD $\$$ (vume): LSET c $\$=$ MKD $\$$ (cond)

LSET D $\$=M K D \$(p s p):$ LSET E\$ = MKD\$(dsp): LSET F\$ = MKD\$(tdp) PUT \#1, I

NEXT I

INPUT "ANY MORE RANGES TO BE CREATED"; ANS\$

1140 OPEN OUTFILE\$ FOR RANDOM AS \#1

1150 FIELD \#1, 12 AS a\$, 12 AS B\$, 12 AS c\$, 12 AS D\$, 12 AS E\$

1160 INPUT "DO YOU WISH A TABLE OF THE DATA. MAKE SURE THE PRINTER IS ON "; ANS\$

1161 IF LEFT\$(ANS\$, 1) = "N" OR LEFT\$(ANS\$, 1) = "n" THEN 1235

1162 INPUT "INPUT RANGE TO BE PRINTED OUT"; IBEG, IEND

1163 INPUT "DO YOU WISH THE PRINTOUT(S) ON THE PRINTER"; ANS\$

1164 IF LEFT\$(ANS\$, 1) = "N" OR LEFT\$(ANS\$, 1) = "n" THEN 1190

1165 LPRINT "\#"; " VOLUME "; "CONDUCTANCE "; "PUMPING SPEED "; "DESORPTION "; "THERMAL DES." 
1166 FOR I = IBEG TO IEND

1167 GET $\# 1,1$

1168 LPRINT 1;

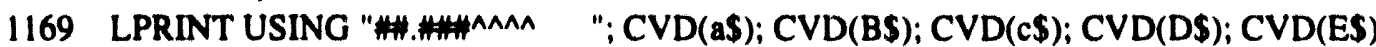

1170 NEXT I

1171 GOTO 1231

1190 PRINT " "\#": " VOLUME $\quad$ ": "CONDUCT. "; "PUMP. SPEED "; "DESORPT. "; "TH. DESORPT."

1192 PRINT

1200 FOR I = IBEG TO IEND

1210 GET $\# 1,1$

1220 PRINT I;

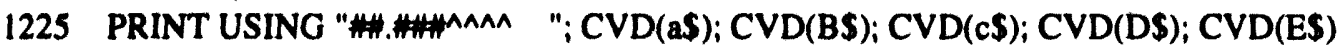

1230 NEXT I

1231 INPUT "ARE THERE ANY MORE RANGES TO BE PRINTED"; ANSS

1232 IF LEFTS(ANSS, 1) = "Y" OR LEFT\$(ANS\$, 1) = "y" THEN 1162

1233 CLOSE \#1

1234 INPUT "ANY ALTERATIONS NEEDED ON FILES"; ANS\$

1235 IF LEFTS(ANSS, 1) = "Y" OR LEFT\$(ANS\$, 1) = "y" THEN 310

1236 PRINT "NO MORE USER INPUTS ARE REQUIRED"

1237 CLOSE \#1

1250 REM *****************************************************

1260 ' THE PROGRAM NOW BEGINS. THE FIRST ORDER OF BUSINESS IS TO CHANGE THE

1270 ' INDIVIDUAL CONDUCTANCES TO CONDUCTANCES BETWEEN THE ELEMENTS USING

$1280^{\prime}$ CONDUCTANCES IN SERIES. NEXT THE FOUR QUANTITIES OF CONDUCTANCE,

1290 ' PUMPING SPEED, VOLUME, DESORPTION, AND THERMAL DESORPTION ARE USED

1300 ' TO SET UP THE MATRIX EQUATION. THE PROGRAM THEN ITERATES THROUGH TIME.

1310 REM ****************************************************

1320 OPEN OUTFILES FOR RANDOM AS \#1

1322 FIELD \#1, 12 AS a\$, 12 AS B\$, 12 AS c\$, 12 AS DS, 12 AS ES

1330 GET \#1, NEM: CURRENT = CVD(B\$)

$1340 \mathrm{~V}(\mathrm{NEM})=\mathrm{CVD}(\mathrm{a} \$): \mathrm{S}(\mathrm{NEM})=\mathrm{CVD}(\mathrm{c} \$): \mathrm{Q}(\mathrm{NEM})=\mathrm{CVD}(\mathrm{D} \$)$

$1345 \mathrm{TD}(\mathrm{NEM})=\mathrm{CVD}(\mathrm{E} \$)$

1350 GET \#1, 1: NEX = CVD(B\$)

$1360 \mathrm{c}(\mathrm{NEM})=$ CURRENT * NEX $/($ CURRENT + NEX)

1370 FOR $I=1$ TO NEM -1

1380 GET \#1, I: CURRENT = CVD(B\$)

$1390 \quad \mathrm{~V}(\mathrm{I})=\mathrm{CVD}(\mathrm{aS})$

$1400 \mathrm{~S}(\mathrm{I})=\mathrm{CVD}(\mathrm{c} \$)$

$1410 Q(I)=C V D(D \$)$

$1411 \mathrm{TD}(\mathrm{I})=\mathrm{CVD}(\mathrm{E} \$)$

1420 GET \#1, I + 1: NEX = CVD(BS)

$1430 \mathrm{c}(\mathrm{I})=$ CURRENT * NEX $/($ CURRENT + NEX)

1440 NEXT I

1450 CLOSE

$1460 \mathrm{~N}=\mathrm{INT}(3600 * \mathrm{TIM} / \mathrm{DT})$

1470 REM *********************************************

1480 ' LABELLING FOR THE LOGARITHMIC GRAPH SHOWING EACH ELEMENTS PRESSURE

$1490 \mathrm{REM} * * * * * * * * * * * * * * * * * * * * * * * * * * * * * * * * * * * * * * * * * * * *$

1500 DATA E,L,E,M,E,N,T,S,

1510 CLS : SCREEN 2

$1520 \mathrm{~L}=\mathrm{INT}(145 / \mathrm{NEM})$

1525 LOCATE 1,1

1630 REM " PRESSURE IN TORR (LOGARITHMIC SCALE STARTING WITH 1.0E-11)"

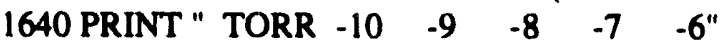




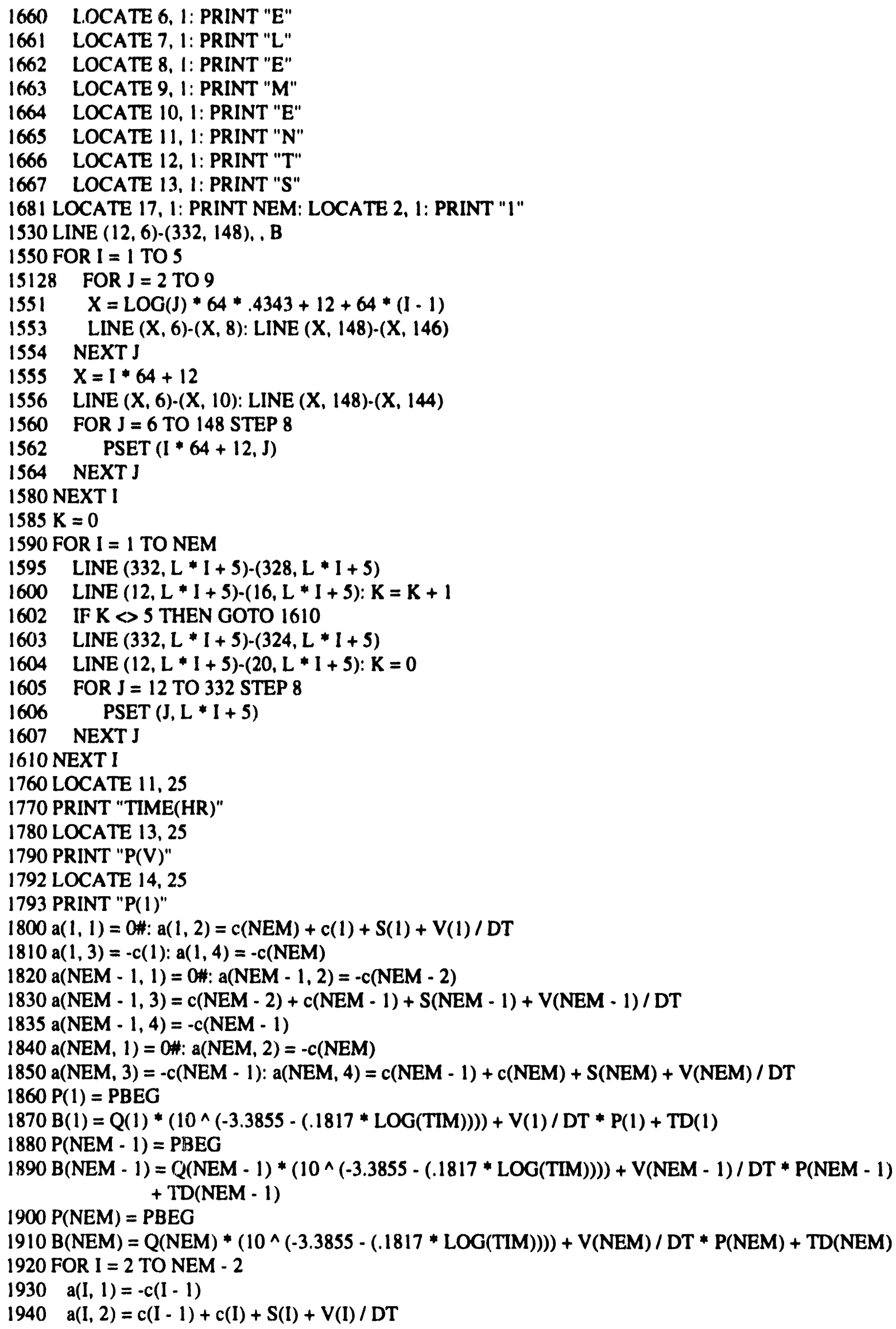




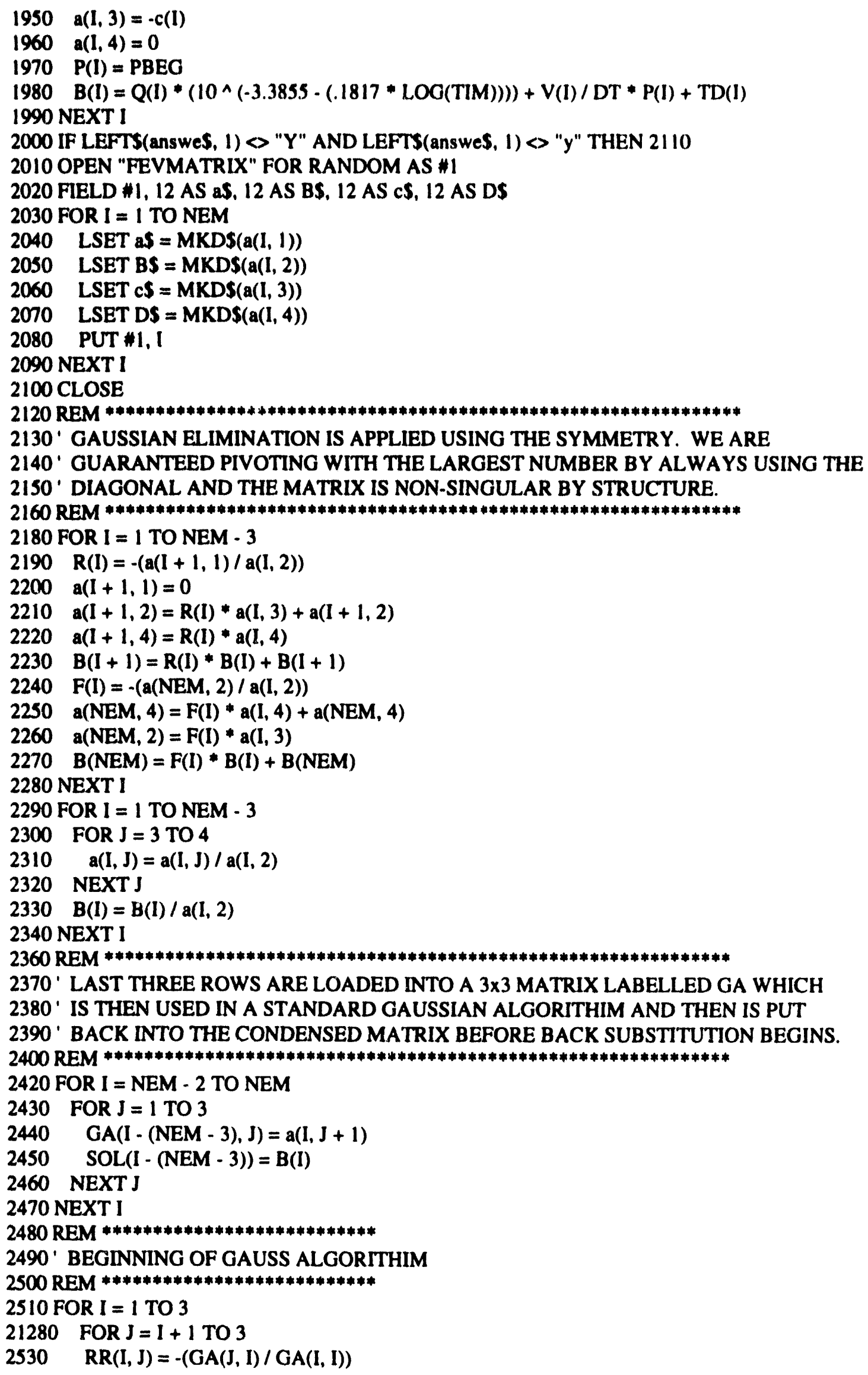




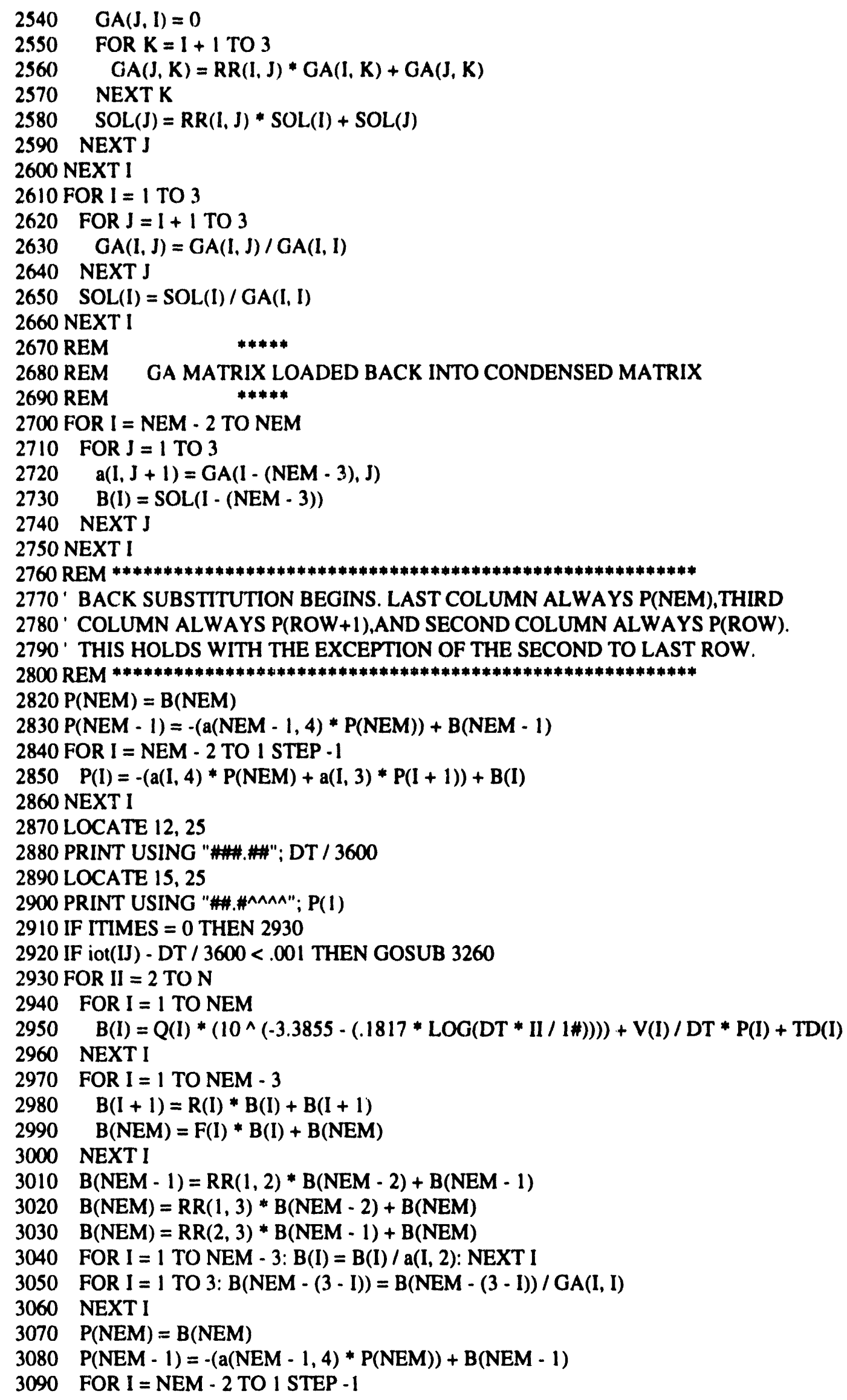




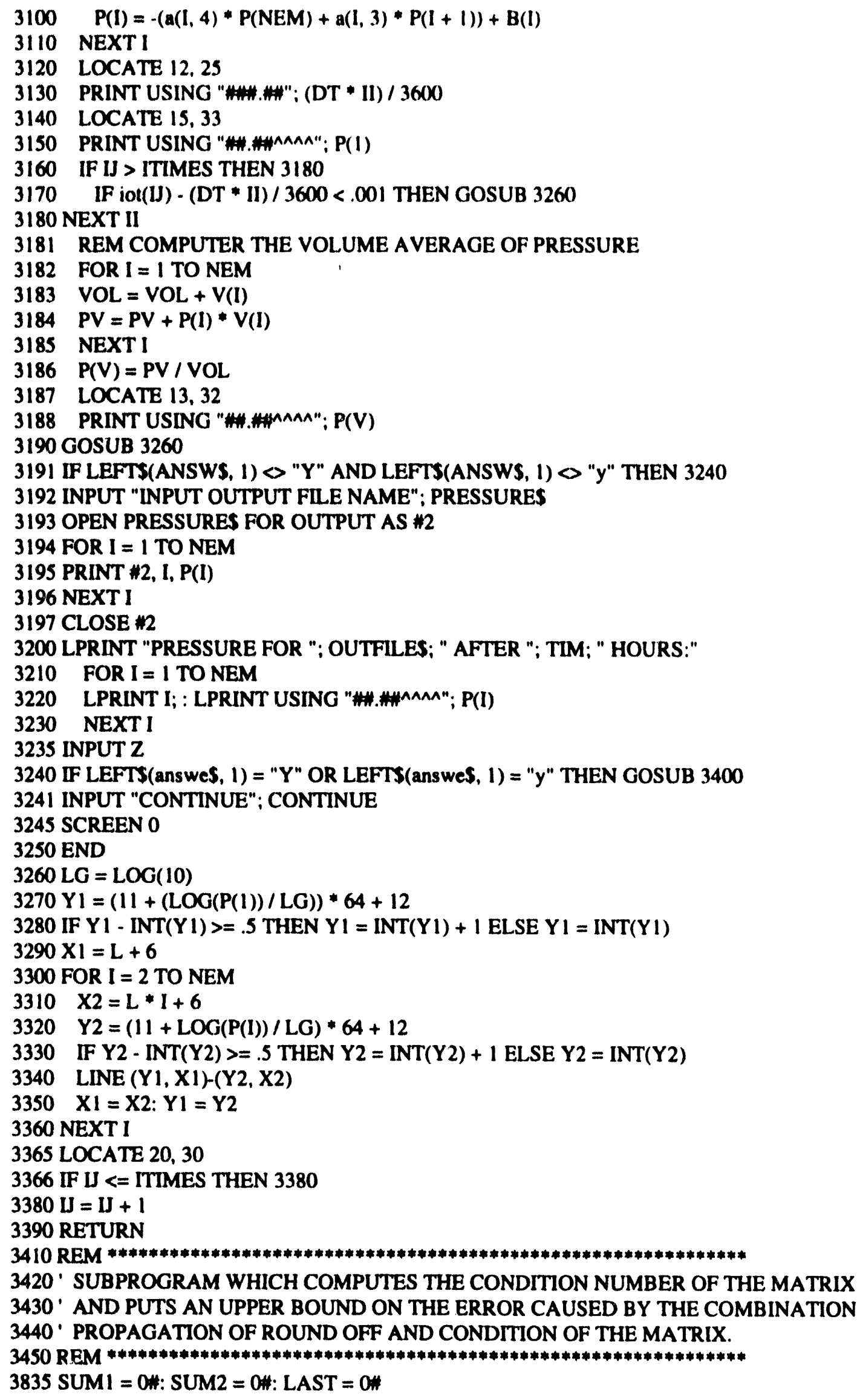




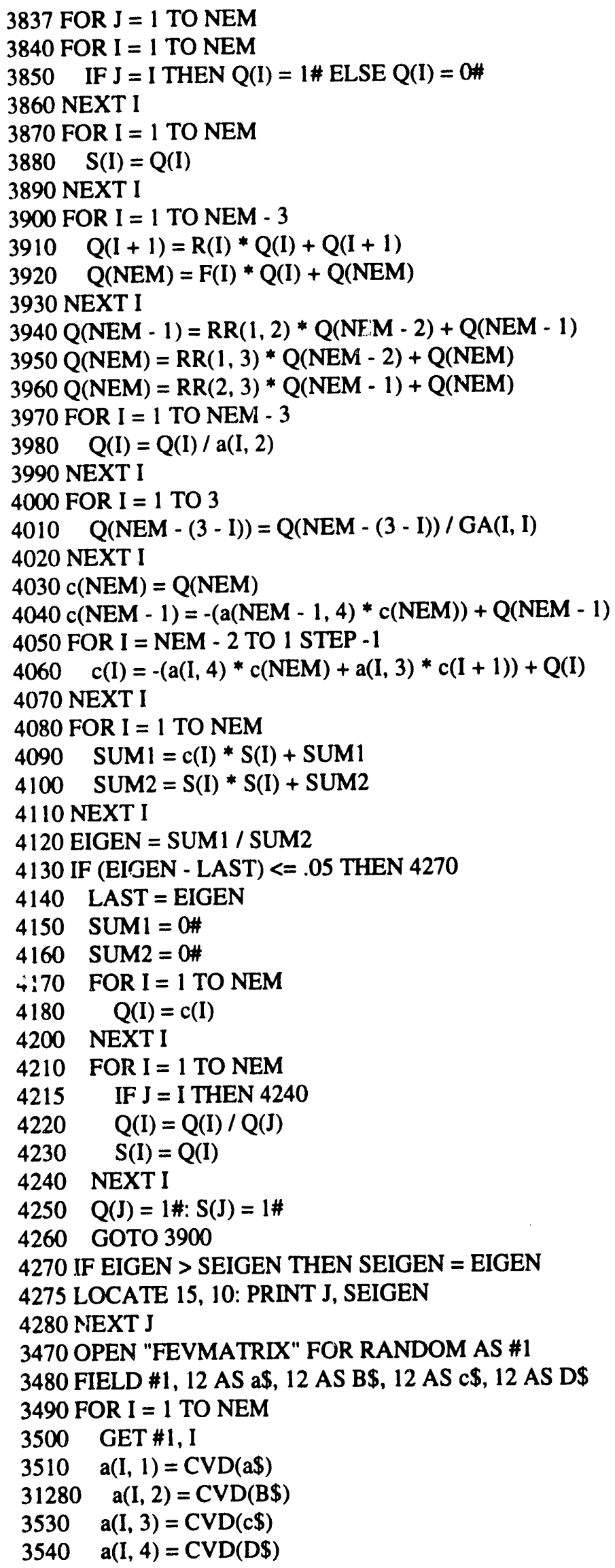




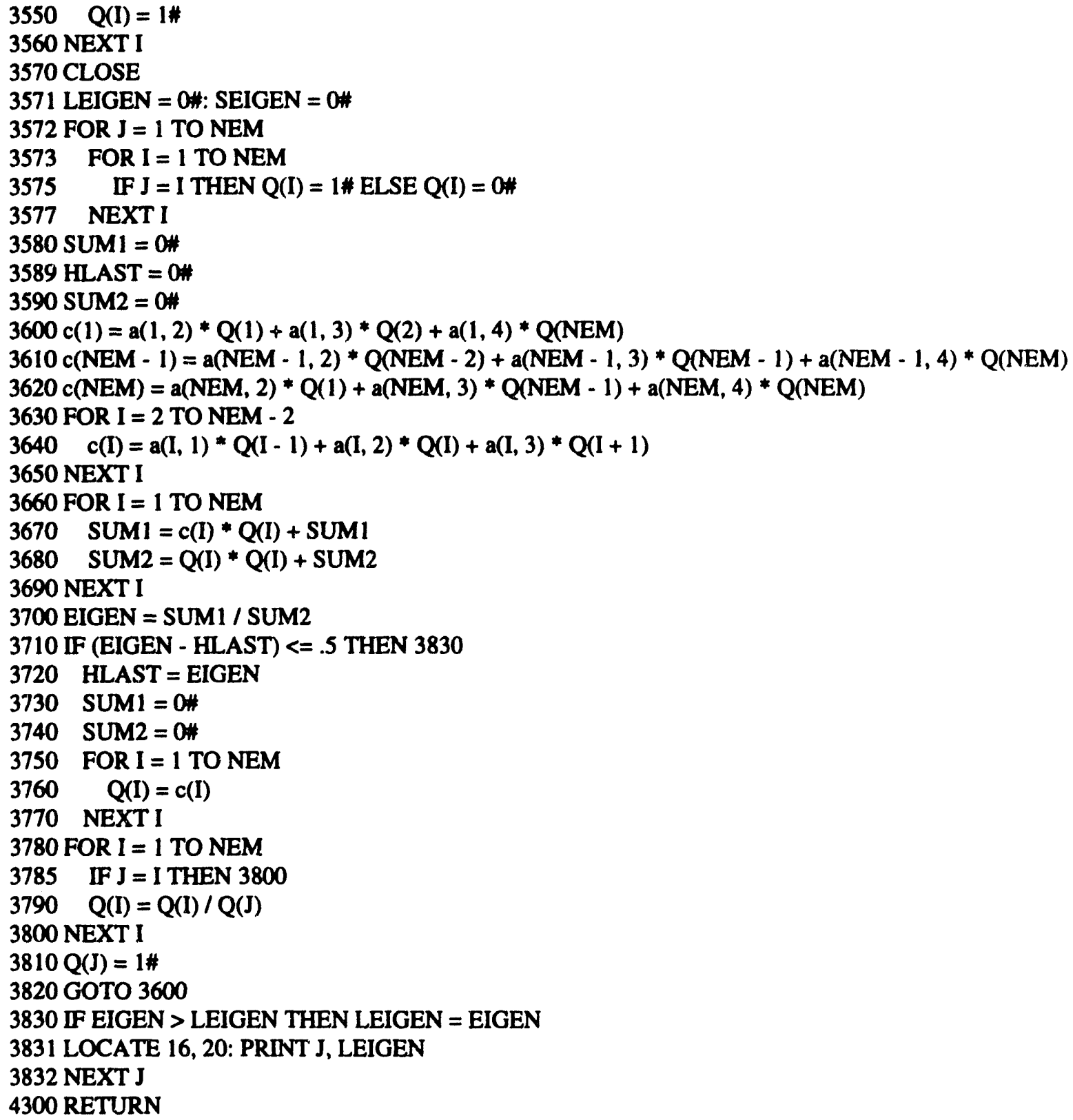



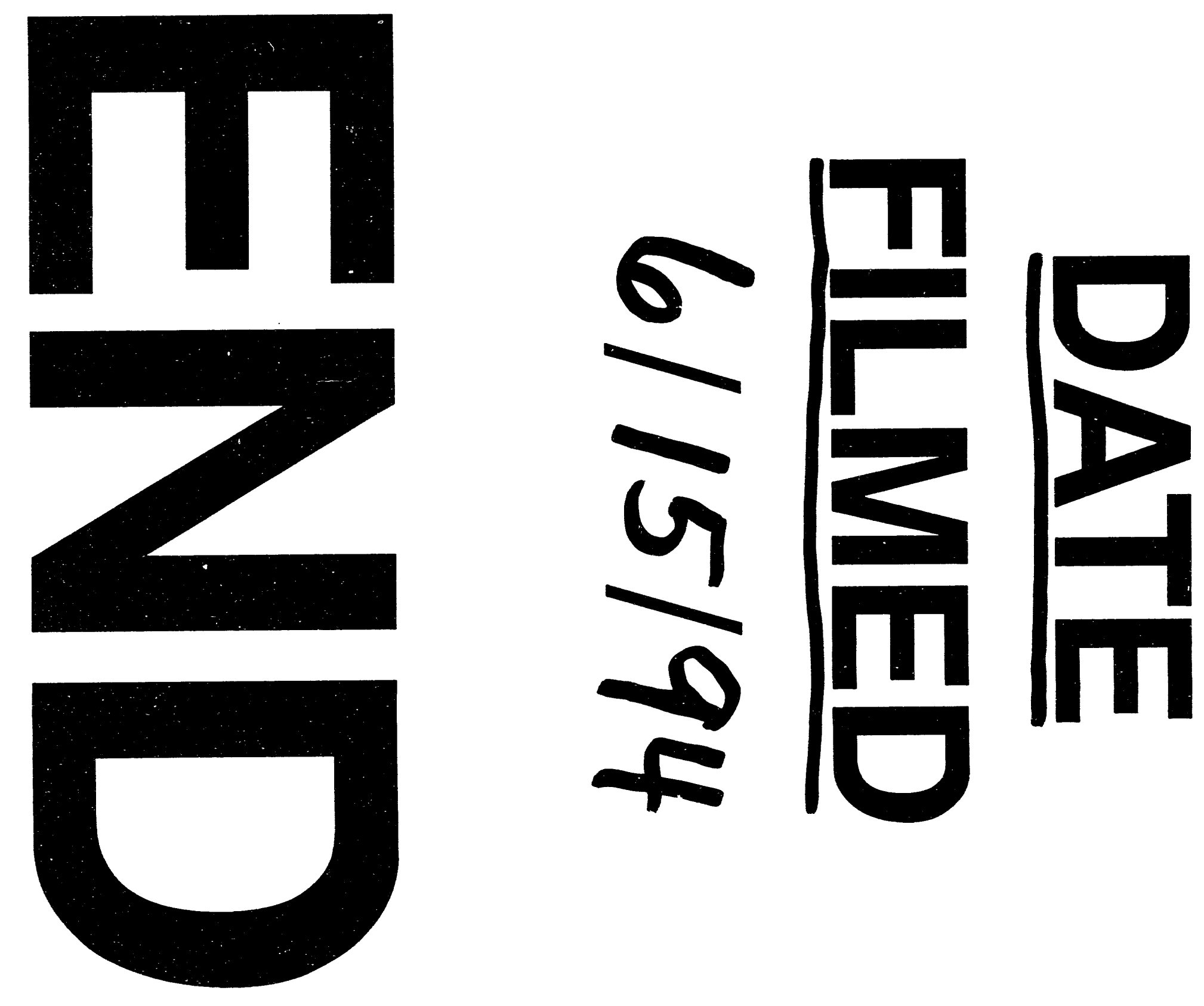
